\title{
Matchings under Preferences: Strength of Stability and Trade-Offs
}

\author{
Jiehua Chen Piotr Skowron \\ Manuel Sorge \\ University of Warsaw, Warsaw, Poland \\ jiehua.chen2@gmail.com p.skowron@mimuw.edu.pl manuel.sorge@gmail.com
}

\begin{abstract}
We propose two solution concepts for matchings under preferences: robustness and near stability. The former strengthens while the latter relaxes the classic definition of stability by Gale and Shapley (1962). Informally speaking, robustness requires that a matching must be stable in the classic sense, even if the agents slightly change their preferences. Near stability, on the other hand, imposes that a matching must become stable (again, in the classic sense) provided the agents are willing to adjust their preferences a bit. Both of our concepts are quantitative; together they provide means for a fine-grained analysis of the stability of matchings. Moreover, our concepts allow the exploration of trade-offs between stability and other criteria of social optimality, such as the egalitarian cost and the number of unmatched agents. We investigate the computational complexity of finding matchings that implement certain predefined trade-offs. We provide a polynomial-time algorithm that, given agent preferences, returns a socially optimal robust matching, and we prove that finding a socially optimal and nearly stable matching is computationally hard.
\end{abstract}

\section{Introduction}

In the Stable Marriage problem [15] we are given two disjoint sets of agents, $U$ and $W$. Each agent from one set has a strict preference list that ranks a subset of the agents from the other set. The sets of agents and their preference lists are collectively called preference profile. The goal is to find a matching - i.e., a bijection between $U$ and $W$ - that does not contain a blocking pair, i.e., a pair of agents who prefer each other over their matched partners. A matching with no blocking pairs is called a stable matching.

The classic definition of stability is qualitative: A matching can be either stable or not, and there are no other states in between or beyond. In this paper, by contrast, we take a quantitative approach. We propose and study two solution concepts: robustness and near stability, where the former strengthens and the latter relaxes the notion of stability. Intuitively, a robust matching is more than stable; it remains stable even if agents change their preferences slightly. In contrast, a nearly stable matching needs not be stable for the original profile, but it becomes so after some minor changes in the preferences. Below we give more precise definitions of robust and nearly stable matchings and motivate their study through a number of observations.

Robust matchings. Our first main observation is that the preference lists provided by the agents do not always reflect their true preferences. This can happen, for instance, because the agents do not 
have full information about their potential partners, or because formulating accurate preferences is a hard task that requires substantial cognitive effort [28]. It is also typical that the agents change their preferences over time, for instance, in response to changes in their operating environment. Thus, a matching that is stable in the classic sense (with respect to the preferences expressed by the agents at the beginning) can in fact contain two or more agents who already have or will likely have incentives to drop their assigned partners and be matched with each other. In other words, there are situations where the classic definition of stability can turn out to be too weak. In a different setting, a third party may want to destabilize a matching by bribing certain agents to change their preferences. In that case, we are interested in stable matchings which defy such attacks.

For the above reasons, we introduce and study d-robustness, a strengthened notion of stability. A matching is $d$-robust for a given preference profile if this matching is stable and remains stable after performing an arbitrary sequence of $d$ swaps. Here, a swap is the reversal of two consecutive agents in a preference list. Intuitively, if a matching is $d$-robust for some reasonably large $d$, then it will not become unstable even if the agents specified slightly inaccurate preferences, nor will it become unstable even if the agents change their preferences by a little. Example 1.1 below illustrates the concept of robustness.

Example 1.1. Consider the profile $P$ below with 4 men and 4 women, where the preference lists are to the right of the corresponding agents; preferences are represented as horizontal lists where more preferred agents are put to the left of the less preferred ones.

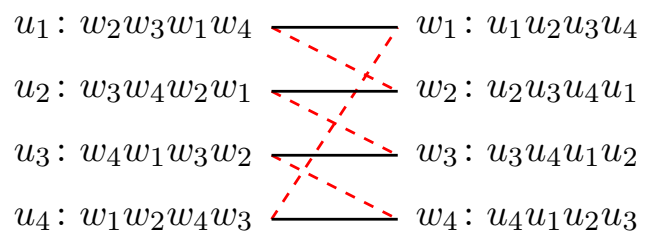

This profile admits five stable matchings:

(1) The $U$-opt. stable matching $M_{1}=\left\{\left\{u_{1}, w_{2}\right\},\left\{u_{2}, w_{3}\right\},\left\{u_{3}, w_{4}\right\},\left\{u_{4}, w_{1}\right\}\right\}$ (red dashed lines),

(2) the $W$-opt. stable matching $M_{2}=\left\{\left\{u_{1}, w_{1}\right\},\left\{u_{2}, w_{2}\right\},\left\{u_{3}, w_{3}\right\},\left\{u_{4}, w_{4}\right\}\right\}$ (black solid lines),

(3) $M_{3}=\left\{\left\{u_{1}, w_{3}\right\},\left\{u_{2}, w_{2}\right\},\left\{u_{3}, w_{1}\right\},\left\{u_{4}, w_{4}\right\}\right\}$,

(4) $M_{4}=\left\{\left\{u_{1}, w_{1}\right\},\left\{u_{2}, w_{4}\right\},\left\{u_{3}, w_{3}\right\},\left\{u_{4}, w_{2}\right\}\right\}$, and

(5) $M_{5}=\left\{\left\{u_{1}, w_{3}\right\},\left\{u_{2}, w_{4}\right\},\left\{u_{3}, w_{1}\right\},\left\{u_{4}, w_{2}\right\}\right\}$.

In terms of robustness, $M_{2}$ is superior to $M_{1}$ since $M_{2}$ is 1-robust but $M_{1}$ is not. To see that $M_{2}$ is 1-robust we observe that, to make $M_{2}$ unstable, we need to perform one swap in the preference list of an agent in $W$. However, no such single swap will make $M_{2}$ unstable. Stable matching $M_{1}$ is not 1-robust since one can swap in the preference list of any agent $u$ from $U$ the two agents $M_{1}(u)$ and $w$ in the first and the second positions to obtain a profile where $\{u, w\}$ is a blocking pair for $M_{1}$. $\diamond$

Nearly stable matchings. Our second main observation is that there exist other factors, apart from the preferences, that can discourage the agents to break their relations with their matched partners. Such factors may include social pressure and additional costs incurred by changing the partner, for example. Thus, in some situations even weaker forms of stability may guarantee a sufficient level of resilience to agents changing their minds. We express this as the local d-near stability of a matching, which stipulates that there is a sequence of swaps such that the matching becomes stable, and in each agent's preference list, at most $d$ swaps are made. 
This concept has an intuitive interpretation similar to the $\epsilon$-Nash-equilibrium [31, Section 2.6.6] in game theory: In a locally $d$-nearly stable matching no agent can improve its satisfaction by more than $d$ through rematching (see also the equivalent definitions in Proposition 2.7). This is analogous to $\epsilon$-Nash-equilibria, where no agent can improve their outcome by more than $\epsilon$. In this sense, local near stability also measures the strength of the incentive for two agents in a blocking pair to change their partners. ${ }^{1}$

Our third main observation is that, when there are constraints on other factors of the matching like social welfare (see below), it may not be possible to find a stable matching satisfying these constraints. Thus, it may be necessary to balance between the social welfare and the costs incurred by agents that want to switch partners. This cost is captured by the global d-near stability of a matching $M$, stating that there is a sequence of at most $d$ swaps in total such that $M$ becomes stable. In order to achieve the desired social welfare, we may thus provide proportionate compensation to the agents affected by the swaps.

Taking nearly stable matchings into consideration may indeed allow us to find a matching that is significantly better from the perspective of the society as a whole, than if we restricted ourselves to stable matchings only. This is illustrated in the following example:

Example 1.2. Let $U=\left\{a_{0}, \ldots, a_{n-1}, x_{1}, \ldots, x_{n}\right\}$ and $W=\left\{b_{0}, \ldots, b_{n-1}, y_{1}, \ldots, y_{n}\right\}$, and consider the following preference profile $P$ of the agents; the index " $i+1$ " is taken modulo $n$.

$$
\begin{array}{lll}
a_{0}: b_{0} b_{1} & b_{0}: a_{0} a_{n-1} & \\
a_{i}: b_{i} b_{i+1} & b_{i}: a_{i-1} x_{1} \ldots x_{n} a_{i} & \text { (for all } i \in\{1, \ldots, n-1\}) . \\
x_{i}: y_{i} b_{1} \ldots b_{n-1} & y_{i}: x_{i} & \text { (for all } i \in\{1, \ldots, n\}) .
\end{array}
$$

In every stable matching of $P$ agent $x_{i}$ must be matched with $y_{i}$ for all $i \in\{1, \ldots, n\}$, and $a_{0}$ with $b_{0}$. Consequently, $a_{1}$ needs to be matched with with $b_{1}$ and, by an inductive argument, we can infer that, for each $i \in\{1, \ldots, n-1\}, a_{i}$ must be matched with $b_{i}$. Thus, if we look at the agents from $B=\left\{b_{1}, \ldots, b_{n}\right\}$, we observe that, except for $b_{0}$, each of them is matched with a partner ranked at the $(n+2)$ th position. Yet, if we consider the profile obtained from $P$ by swapping $a_{0}$ and $a_{n-1}$ in the preference list of $b_{0}$, then $M=\left\{\left\{a_{0}, b_{1}\right\},\left\{a_{1}, b_{2}\right\}, \ldots,\left\{a_{n-1}, b_{0}\right\}\right\} \cup\left\{\left\{x_{i}, y_{i}\right\} \mid 1 \leq i \leq n\right\}$ would be a stable matching. In this matching everyone is matched to one of her two most preferred agents. $\diamond$

Intuitively, Example 1.2 shows that with a relatively small loss of stability, one can significantly improve the social cost of a matching $M$-in this example, this cost is defined as the sum of ranks that an agent $a$ has in the preference list of its matched partner $M(a)$. In the literature this measure is often referred to as the egalitarian cost of a matching [20]. We also consider another metric that counts the number of agents that are assigned a partner in a matching. Recall that we assume that the preference rankings of the agents are incomplete: the agents do not rank those from the opposite set that they would not agree to be matched to. In such a case a stable matching does not need to be perfect, i.e., it is possible that some agents will not be matched at all. The effect of stability on the number of matched agents is illustrated in Example 1.3.

Example 1.3. Consider a profile with 2 men, $a_{1}$ and $a_{2}$, and 2 women, $b_{1}$ and $b_{2}$, with preference lists: $a_{1}: b_{1} ; \quad b_{1}: a_{2} a_{1} ; \quad a_{2}: b_{1} b_{2} ; \quad b_{2}: a_{2}$. For this profile, the only stable matching is $\left\{\left\{a_{2}, b_{1}\right\}\right\}$. However, if we swapped $b_{1}$ and $b_{2}$ in the preference list of $a_{2}$, then $\left\{\left\{a_{1}, b_{1}\right\},\left\{a_{2}, b_{2}\right\}\right\}$ would be a stable matching, i.e., we would obtain a stable matching where more agents have partners. $\diamond$

\footnotetext{
${ }^{1}$ There are some differences between the two concepts since we deal with ordinal preferences. Yet, our concepts generalize to cardinal utilities, where the similarities are more transparent.
} 
Table 1: Summary of our results, where $d$ denotes the number of swaps for robust matchings, $\mathrm{d}_{\mathrm{L}}$ (resp. $d_{G}$ ) denotes the number of swaps for global near stability (resp. local near stability), $\eta$ denotes the egalitarian cost of the desired matching, and $n_{u}$ denotes the number of unmatched agents in any stable matching of the initial profile without ties.

\begin{tabular}{|c|c|c|c|c|}
\hline $\begin{array}{l}\text { Social } \\
\text { criteria }\end{array}$ & $\begin{array}{c}\text { Robust } \\
\text { (without ties) }\end{array}$ & $\begin{array}{c}\text { Robust } \\
\text { (with ties) }\end{array}$ & $\begin{array}{l}\text { Globally Nearly Stable } \\
\text { (without ties) }\end{array}$ & $\begin{array}{l}\text { Locally Nearly Stable } \\
\text { (without ties) }\end{array}$ \\
\hline $\begin{array}{l}\text { No further } \\
\text { restrictions }\end{array}$ & $\begin{array}{c}\mathrm{P} \\
{[\mathrm{Thm} 3.21]}\end{array}$ & $\begin{array}{l}\text { NP-h }(d=1) \\
\quad[\text { Thm 4.1] }\end{array}$ & \multicolumn{2}{|c|}{$\begin{array}{c}\text { Always exists even for } \mathrm{d}_{\mathrm{G}}=\mathrm{d}_{\mathrm{L}}=0 \\
\text { and can be found in } O\left(n^{2}\right) \text { time }[15,18]\end{array}$} \\
\hline $\begin{array}{l}\text { Perfect } \\
\text { matching }\end{array}$ & $\begin{array}{c}\mathrm{P} \\
{[\mathrm{Thm} 3.24]}\end{array}$ & $\begin{array}{c}\text { NP-h }(d=0) \\
{[25]}\end{array}$ & $\begin{array}{l}\text { XP for } \mathrm{d}_{\mathrm{G}}, \mathrm{W}[1]-\mathrm{h} \text { for } \mathrm{n}_{\mathrm{u}}[\text { Cor } 5.6] \\
\text { No poly-approximation [Thm 5.3] }\end{array}$ & $\begin{array}{c}\text { NP-h }\left(\mathrm{d}_{\mathrm{L}}=1\right)[\text { Thm } 5.3], \mathrm{W}[1]-\mathrm{h} \text { for } \mathrm{n}_{\mathrm{u}}[\text { Cor } 5.6] \\
\text { No poly-approximation }[\text { Thm } 5.3]\end{array}$ \\
\hline $\begin{array}{l}\text { Egalitarian } \\
\text { cost } \eta\end{array}$ & $\begin{array}{c}\mathrm{P} \\
{[\text { Thm 3.24] }}\end{array}$ & $\begin{array}{c}\text { NP-c }(d=0) \\
{[25]}\end{array}$ & $\begin{array}{c}\text { XP for } \mathrm{d}_{\mathrm{G}}, \mathrm{W}[1]-\mathrm{h} \text { for } \mathrm{n}_{\mathrm{u}} \\
\text { No poly-approximation }[\mathrm{Thm} 5.3]\end{array}$ & $\begin{array}{l}\text { NP-h }\left(\mathrm{d}_{\mathrm{L}}=1\right)\left[\text { Thm 5.3], W[1]-h for } \mathrm{n}_{\mathrm{u}}\right. \\
\text { No poly-approximation }[\text { Thm 5.3] }\end{array}$ \\
\hline
\end{tabular}

Examples 1.2 and 1.3 suggest that there is a (possibly non-linear) trade-off between stability and other criteria of social optimality. Our definition of near stability provides a formalism necessary to describe the trade-offs; yet, in order to take advantage of them, one needs to be able to identify situations where a large improvement of social welfare is possible with a relatively small sacrifice of stability. We formalize this question as a computational problem (see Section 2.1 for formal definitions) and study its complexity.

Our contributions. We introduce the concepts of robustness and near stability, and explore the trade-off between stability and the egalitarian cost and between stability and the number of matched agents. We provide a polynomial-time algorithm that, given a preference profile and a number $d$, finds a matching which is $d$-robust if it exists (Theorem 3.21). We achieve this by providing a polynomial-size characterization of the profiles (Section 3.2) which are close to the input profile and by heavily exploiting the structural properties of so-called rotations adherent to a preference profile [18]. Moreover, we provide a polynomial-time algorithm that finds a $d$-robust matching with minimum egalitarian cost if one exists (Theorem 3.24). However, when ties are present, we show that finding a robust matching is NP-hard (Theorem 4.1).

In contrast to the polynomial-time algorithms for robust matchings, we show that the problem of finding a matching that implements a certain trade-off between the near stability and the egalitarian cost, or between the near stability and the perfectness of the matching is NP-hard, and it is NP-hard to approximate (Theorem 5.3). Motivated by this general hardness result, we study the parameterized complexity, mainly with respect to the parameter number of allowed swaps (for details on parameterized complexity we refer to the books of Cygan et al. [10], Downey and Fellows [11], Flum and Grohe [14], and Niedermeier [30]). See Table 1 for a summary. Unfortunately, we mostly obtain further hardness results. While for local near stability even only one allowed swap leaves the problem NP-hard (Theorem 5.3), for global near stability there is a polynomial-time algorithm for each constant number $d_{G}$ of allowed swaps (Proposition 5.4). The exponent in the running time depends on $\mathrm{d}_{\mathrm{G}}$, however, and this dependency cannot be removed unless the unlikely complexity-theoretic collapse FPT $=\mathrm{W}[1]$ happens (Theorem 5.5). We also study the complexity in the cases where there are small numbers of unmatched or matched agents in a classically stable matching of the input profile. 
Related work. For an overview on the Stable MARRiage and related problems, we refer to the books of Knuth [22], Gusfield and Irving [18], and Manlove [26].

First, we review work related to our concept of robustness. As we mentioned in the beginning of this section, one of the observations that motivates our study of robust matchings is that the preferences of the agents may be uncertain. In this regard, Aziz et al. [2], Miyazaki and Okamoto [29], and Chen et al. [9] study a variant of STABLE MARRIAGE where there is a collection of "possible" preference profiles given as input, and they look for a matching that is stable in each of the given profiles (the corresponding computational problem is NP-hard even for constant number of input profiles). Our work starts with the assumption that the preferences provided by the agents are a good approximation of their true preferences. Thus, our robustness concept respects every profile that is close to the preferences provided by the agents. This makes a crucial difference-finding a robust matching if one exists, according to our definition, is solvable in polynomial time.

Our robustness concept is related to the works of Mai and Vazirani [23, 24]. They introduced a probabilistic model, where there are polynomially many preference profiles given in the input, each differing from the original one by a single agent's preference list. While they do not assume this difference to be small, they assume there is a probabilistic distribution over these polynomially many preference profiles, and the goal is to find a stable matching that stays stable with the highest probability. In contrast, in our definition of robustness, we require that the sought matching must be stable in every profile which is close to the original one, but which can differ from the original profile in preference lists by more than one agent. Furthermore, we do not assume that the distribution of the profiles is given, but rather infer the "relevant" (close) profiles directly from the original profile. Our approach, based on the concept of distances, induces a quantitative measure of the strength of stability; we further extend it in the converse direction by considering matchings that are nearly stable, getting a full set of tools that allow to reason about the strength of stability for any matching.

Mai and Vazirani [23, 24] proved that a matching that stays stable with the largest probability for a given probability distribution can be found in polynomial time as long as only a single agent changes her preferences in each profile. Their techniques crucially rely on the fact that, for each of the preference profiles $P^{\prime}$ that has nonzero probability, the set of matchings that are stable for both $P^{\prime}$ and the input profile $P$ has a certain type of sublattice structure of the lattice of stable matchings of $P$. In our model, this is not the case; the set of stable matchings for the preference profiles obtained by $d$ arbitrary swaps may not be a sublattice anymore. Thus, this approach is not directly applicable in our scenario. Moreover, in the general case, where we allow arbitrary changes between profiles, it is impossible to obtain a polynomial-time algorithm unless $\mathrm{P}=\mathrm{NP}$ : Miyazaki and Okamoto [29] and Chen et al. [9] showed that finding a matching which is stable for even only two profiles is NP-hard. Nevertheless, we provide a compact characterization of all close profiles, based on which, and partly inspired by the techniques of Mai and Vazirani [23], we provide a polynomial-time algorithm for our robustness model. In this regard, our algorithmic techniques can be considered as a generalization of the ones by Mai and Vazirani [23].

Finally, let us mention a relation between robustness and strategy-proofness. We say that a matching algorithm is strategy-proof (see [18, Chapter 4], [33, Chapter 1.7], [26, Chapter 2.9]) if no agent can obtain a better partner by misreporting her preferences; it is known that there exists no strategy-proof matching mechanism. Robustness implies a very weak form of strategyproofness, where the set of agents' strategies is limited - the agents are willing to report only those rankings that are not significantly different from their true preferences. Even more closely related, robustness implies resilience to certain forms of bribery - the problem of bribery, originally defined 
for single-winner elections [13], can be naturally adapted to matchings.

Now we turn to work that is more related to our near stability concept. Another interpretation of a locally $d$-nearly stable matching is that in each blocking pair there is an agent whose rank improvement by switching partners would be at most $d$. Drummond and Boutilier [12] use this rank improvement approach to study STABLE MARRIAGE problem under partially ordered preferences. They introduced the notion of an $r$-maximally stable matching, i.e., a matching such that for each linear completion of the input profile and for each unmatched pair at least one agent in the pair ranks the other higher than its matched partner by at most $r$ positions. When restricting the input to linear preferences, as is our focus here, $r$-maximal stability is equivalent to local $r$-near stability for each $r \geq 0$. We prove this formally in Proposition 2.7. Here, in contrast, we do not deal with partial preferences, but instead we want to achieve a given social welfare in addition to $r$-maximal stability.

Pini et al. [32] and Anshelevich et al. [1] studied a concept called (additive) $\alpha$-stability that measures the degree of instability for utility-based preferences. For ordinal preferences, their concept is equivalent to our local $\alpha$-near stability. Anshelevich et al. [1] studied the trade-off between the total utility of a matching and its $\alpha$-stability for restricted structures of utility scores (which cannot model ordinal preferences). Pini et al. [32] showed that a certain kind of lexicographically optimal $\alpha$-stable matching can be found in polynomial time and they considered manipulation issues.

Finally, we review further related work, not necessarily directly related to our notions of robustness or near stability. Recently, Menon and Larson [27] proposed a different robustness concept to deal with uncertain preferences-the authors assume that each agent has preferences with ties on the agents of the opposite set and look for a perfect matching so as to minimize the maximum number of blocking pairs among all linear extensions of the input preferences. In contrast to our approach, however, these blocking pairs may represent an arbitrarily large rank improvement, i.e. an arbitrarily large number of swaps needed to make the matching stable. Finding a solution as above is equivalent to finding a perfect matching with minimum number of so-called super-blocking pairs, a concept introduced by Irving [19] to cope with preferences with ties, i.e. weak orders (also see [18]). Menon and Larson [27] mainly obtained inapproximability results.

Genc et al. $[16,17]$ provide yet another view on robustness in the context of stable matchings. They define an $(x, y)$-supermatch as a stable matching that satisfies the following property: If any $x$ agents break up, it is possible to rematch these $x$ agents so that the new matching is again stable; further, this re-matching must be done by breaking at most $y$ other pairs. Hence, an $(x, y)$-supermatch may not be robust in our sense, but it needs to be easy to repair.

In the second part of this paper we study trade-offs between the stability (of various strength) and other criteria of optimality such as the egalitarian cost and the number of unmatched agents. This is related to the studies on the price of stability in matching markets [4]. Concepts similar to our robustness have been also studied in other contexts, for instance for single-winner [34] and multi-winner elections [6].

\section{Basic Definitions, Notations, and Our Stability Concepts}

For each natural number $t$ by $[t]$ we denote the set $\{1,2, \ldots, t\}$. Let $U=\left\{u_{1}, \ldots, u_{n}\right\}$ and $W=\left\{w_{1}, \ldots, w_{n}\right\}$ be two $n$-element disjoint sets of agents. A preference profile $P=\left(\left(\succ_{u}^{P}\right)_{u \in U},\left(\succ_{w}^{P}\right)_{w \in W}\right)$ is a collection of the preference lists of the agents from $U$ and $W$. Here, for each agent $u \in U$, the notation $\succ_{u}^{P}$ denotes a linear order on a subset $W^{\prime}$ of $W$ that represents the ranking of agent $u$ over all agents from $W^{\prime}$ in profile $P$. The agents in $W^{\prime}$ 
are also called acceptable to $u$. The candidates not ranked by $u$ are those in $W \backslash W^{\prime}$, that is, those that $u$ does not agree to be matched to; we also call them unacceptable. If $w \succ_{u}^{P} w^{\prime}$ then we say that $w$ is preferred to $w^{\prime}$ by $u$ in $P$. Analogously, for each agent $w \in W, \succ_{w}$ represents a linear order on (a subset of) $U$ that represents the ranking of $w$ in profile $P$ and we likewise use the notions of preference list, preferred, (un-)acceptable, and (not) ranked. We say that $P$ has complete preferences if each agent finds all agents from the opposite set acceptable.

Given an agent $x$ with her preference list $\succ_{x}$ and given an agent $y$ from the opposite set, the rank $\mathrm{rk}_{x}\left(y, \succ_{x}\right)$ of $y$ in the preference list of $x$ is equal to the number of agents that are preferred to $y$ by $x$. If $y$ is not acceptable to $x$ then we let $\mathrm{rk}_{x}\left(y, \succ_{x}\right)$ be equal to the number of agents acceptable to $x$. We usually omit the symbol $\succ_{x}$ in $\operatorname{rk}_{x}\left(y, \succ_{x}\right)$ and write only $\operatorname{rk}_{x}(y)$ whenever the preference list of $x$ is clear from the context. For instance, the rank of $y_{3}$ in the preference list $\succ_{x}: y_{1} \succ y_{3} \succ y_{2}$ is one. We say that $x$ ranks $y$ higher than $z$, if $\mathrm{rk}_{x}(y)<\mathrm{rk}_{x}(z)$.

Throughout, except in Section 4 , by " $x \succeq y$ " for two agents $x$ and $y$ we mean " $x=y$ or $x \succ y$ ".

Blocking pairs and stable matchings. Given two disjoint sets of agents, $U$ and $W$, a matching $M$ is a set of pairwise disjoint pairs, each pair containing one agent from $U$ and one agent from $W$, i.e. $M \subseteq\{\{u, w\} \mid u \in U \wedge w \in W\}$ and for each two pairs $p, p^{\prime} \in M$ it holds that $p \cap p^{\prime}=\emptyset$. Given a pair $\{u, w\}$ with $u \in U$ and $w \in W$, if it holds that $\{u, w\} \in M$, then we use $M(u)$ to refer to $w$ and $M(w)$ to refer to $u$, and we say that $u$ and $w$ are their respective partners under $M$; otherwise we say that $\{u, w\}$ is an unmatched pair. We say that $\{u, w\}$ is blocking (or a blocking pair of ) $M$ if both $u$ and $w$ would prefer to be matched together than to stay with their current partners. Formally, $\{u, w\}$ is a blocking pair if the following holds: (1) $u$ and $w$ find each other acceptable but are not matched together, $(2) u$ is either unmatched by $M$ or $\mathrm{rk}_{u}(w)<\mathrm{rk}_{u}(M(u))$, and (3) $w$ is either unmatched by $M$ or $\mathrm{rk}_{w}(u)<\mathrm{rk}_{w}(M(w))$. We say that a matching $M$ is stable if no unmatched pair forms a blocking pair for $M$. Example 1.1 in the introduction illustrates stable matchings.

We use $\mathcal{S M}(P)$ to denote the set of all stable matchings for a preference profile $P$. Given a matching $M$, we use $\mathcal{B P}(P, M)$ to denote the set of all unmatched pairs that are blocking $M$ in profile $P$. Obviously, for each stable matching $M \in \mathcal{S M}(P)$, it holds that $\mathcal{B P}(P, M)=\emptyset$.

\subsection{Our Spectrum of Stability Notions and Problems}

Let us now define our concepts of robustness and near stability, informally introduced in Section 1.

First of all, we need the notion of swaps, which describes the operation of taking two consecutive agents $x$ and $y$ in a preference list of a third agent $z$ and switching their relative order in order to obtain a new preference list. We also use $(z,\{x, y\})$ to denote such a swap. Given two preference lists $\succ$ and $\succ^{\prime}$, the swap distance (also known as the Kendall $\tau$ distance [21]) between $\succ$ and $\succ^{\prime}$ is defined as the number of differently ordered pairs in the two lists; if the two lists are defined on two different acceptable sets, then the swap distance is infinity. Intuitively, the swap distance is equal to the minimum number of swaps that are required to turn $\succ$ into $\succ^{\prime}$. Accordingly, the swap distance between two preference profiles $P_{1}$ and $P_{2}$, denoted as $\tau\left(P_{1}, P_{2}\right)$, is defined as the sum of swap distances between the two preference lists of each agent in profiles $P_{1}$ and $P_{2}$.

Definition 2.1 (Robustness). For a given preference profile $P$, we say that a matching $M$ is $d$-robust if for each profile $P^{\prime}$ with $\tau\left(P, P^{\prime}\right) \leq d$ it holds that $M$ is stable in $P^{\prime}$. 
Note that our robustness concept is monotone-each $d$-robust matching is also $d^{\prime}$-robust for $0 \leq d^{\prime} \leq d$. We are interested in the computational question of finding the maximal integer $d$ such that there is a $d$-robust matching. This can be phrased as a decision problem as follows:

\section{Robust Matching}

Input: A preference profile $P$ with agent sets $U$ and $W$ of size $n$ each, and an integer $d \in \mathbb{N}$.

Question: Is there a $d$-robust matching for $P$ ?

Now, we define near stability. Here, we provide two definitions - global near stability and local near stability - that differ in the scope of admissible changes to the original preference profile.

Definition 2.2 (Near stability). For a given preference profile $P$, we say that a matching $M$ is globally $d$-nearly stable if there exists a profile $P^{\prime}$ with $\tau\left(P, P^{\prime}\right) \leq d$ such that $M$ is stable in $P^{\prime}$. We say that $M$ is locally $d$-nearly stable if there exists a profile $P^{\prime}$ with $\tau\left(\succ_{x}^{P}, \succ_{x}^{P^{\prime}}\right) \leq d$ for each agent $x \in U \cup W$ such that $M$ is stable in $P^{\prime}$.

Since near stability is a more permissive concept than stability as defined by Gale and Shapley [15], it is straight-forward that a globally $d$-nearly stable (or locally $d$-nearly stable) matching always exists for $d \geq 0$. Here, our main focus is to explore the trade-offs between the strength of stability and other criteria of social optimality. We say that a matching $M$ is perfect if each agent has a partner in $M$. The egalitarian cost of $M$ in a profile $P=\left(\succ_{x}\right)_{x \in U \cup W}$ is $\eta(M):=\sum_{x \in U \cup W} \mathrm{rk}_{x}\left(M(x), \succ_{x}\right)$. This leads to the following computational problems, abbreviated as Global-Near+Egal, and Local-NeAR+Egal.

Globally (or Locally) Nearly Stable Perfect Matching

Input: A preference profile $P$ with agent sets $U$ and $W$ of size $n$ each, and an integer $d \in \mathbb{N}$.

Question: Is there a globally $d$-nearly stable (or locally $d$-nearly stable) stable matching for $P$ which is perfect?

\section{Globally (or Locally) Nearly Stable Egalitarian Matching}

Input: A preference profile $P$ with agent sets $U$ and $W$ of size $n$ each, and two integers $d, \eta \in \mathbb{N}$. Question: Is there a globally $d$-nearly stable (or locally $d$-nearly stable) stable matching for $P$ which has egalitarian cost at most $\eta$ in $P$ ?

For preferences without ties (i.e. every agent has a strict preference list), we will use the following fundamental result from the literature.

Proposition 2.3 ([18, Theorem 1.4.2]). For incomplete preferences without ties, the agent set can be partitioned into two disjoint subsets $R$ and $S$ such that every stable matching matches every agent from $R$ and none of the agents from $S$. For agent set of size $2 n$, this partition can be computed in $O\left(n^{2}\right)$ time. 


\subsection{Structural Properties of Robust and Nearly Stable Matchings}

Before we proceed further, we provide some structural results concerning Definitions 2.1 and 2.2. First we give two observations regarding robustness. These are not necessary for the considerations about algorithms later on, but serve to strengthen the intuition about profiles that allow for robust matchings and, we feel, are interesting in their own right. Further below, we consider the trade-off between near stability and perfectness of matchings and give alternative characterizations of locally nearly stable matchings.

Proposition 2.4. If $d \geq n$ and there exists one agent who finds at least two other agents acceptable, then no stable matching is d-robust.

Proof. Let $M$ be an arbitrary stable matching. To show that no stable matching is $d$-robust, it suffices to show that performing at most $n$ swaps can make an unmatched pair a blocking pair of $M$. To this end, let $x$ be an agent who finds at least two other agents acceptable. Further, let $y$ be an acceptable agent of $x$, satisfying the following. If $x$ is unmatched under $M$ or if $\operatorname{rk}_{x}(M(x)) \geq 1$, then $y$ is the most preferred agent of $x$ (that is, $\mathrm{rk}_{x}(y)=0$ ); otherwise, $y$ is the second-most preferred agent of $x$ (that is, $\operatorname{rk}_{x}(y)=1$ ). Now, use at most one swap to make agent $y$ the most preferred agent of $x$, and at most $n-1$ swaps to make agent $x$ the most preferred agent of $y$. This results in $\{x, y\}$ being a blocking pair of $M$. Hence, $M$ is not $d$-robust, as $d \geq n$.

A matching is top-choice if each agent is matched to her most preferred partner. A profile is position-wise distinct if there are no two agents that have the same agent in the same position in their preference lists.

Proposition 2.5. Every $(n-1)$-robust matching is top-choice and every profile allowing for an $(n-1)$-robust matching is position-wise distinct.

Proof. Let $P$ be a preference profile on two $n$-element sets $U$ and $W$, and let $M$ be an $(n-1)$ robust matching. We first show that $M$ is top-choice. This is clear if each agent finds only one other agent acceptable. Otherwise, there is at least one unmatched pair of agents. Observe that for each unmatched pair $\{x, y\}$ of agents it must hold that

$$
\mathrm{rk}_{x}(y)+\mathrm{rk}_{y}(x) \geq n
$$

as otherwise we can perform at most $n-1$ swaps, $\mathrm{rk}_{x}(y)$ swaps in $x$ 's preference list and $\mathrm{rk}_{y}(x)$ swaps in $y$ 's preference list, to make $x$ and $y$ be each other's most preferred agent. This results in $\{x, y\}$ being a blocking pair of $M$ - a contradiction to $M$ being $(n-1)$-robust.

To show that $M$ is top-choice, towards a contradiction, suppose that $M$ is not top-choice. This means that there exists an unmatched pair $\{x, y\}$ such that $y$ is the most preferred agent of $x$, i.e. $\mathrm{rk}_{x}(y)=0$. However, by (1), it implies that $\mathrm{rk}_{y}(x) \geq n$ - a contradiction to the fact that the rank of an agent is at most $n-1$.

It remains to prove that $P$ is position-wise distinct. We first consider the case when $n=2$ and then the case when $n \geq 3$; the case with $n=1$ is trivial. Assume that $n=2$. Since $M$ is top-choice, we infer that the most preferred agents of two different agents are different from each other. Now suppose, for the sake of contradiction, that there are three distinct agents $x_{1}, x_{2}, y$ such that $\mathrm{rk}_{x_{1}}(y)=1$ and $\mathrm{rk}_{x_{2}}(y)=1$. By (1), we must have that $\mathrm{rk}_{y}\left(x_{1}\right) \geq n-1=1$ and $\mathrm{rk}_{y}\left(x_{2}\right) \geq n-1=1$. Hence, $\mathrm{rk}_{y}\left(x_{1}\right)=\mathrm{rk}_{y}\left(x_{2}\right)=1$ as each rank is at most $n-1=1$, this is a 
contradiction to the fact that no two agents have the same rank by the same agent. This finishes the proof for the case when $n=2$.

In the remainder of the proof, we assume that $n \geq 3$. Before proving that $P$ is position-wise distinct in this case as well, we observe that each agent finds all other agents acceptable.

Let $u$ and $w$ be two unmatched agents such that $\mathrm{rk}_{u}(w)=1$. By $(1)$, we have $\operatorname{rk}_{w}(u) \geq n-1$ and indeed $\mathrm{rk}_{w}(u)=n-1$ since $n-1$ is the largest-possible rank. This implies that $w$ appears in the preference list of every agent from $U$. By the top-choice property of $M$, we infer that, except for the partner $M(w)$ of $w$, every agent $x$ from $U \backslash\{M(w)\}$ finds at least two agents acceptable: her partner $M(x)$ and agent $w$. We claim that indeed $M(w)$ also finds at least two other agents acceptable. Since $n \geq 3$, there is a third agent $u^{\prime} \in U \backslash\{M(w), u\}$ who finds $w$ acceptable. Let $w^{\prime} \in$ $W$ be an agent with $\mathbf{r k}_{u^{\prime}}\left(w^{\prime}\right)=1$. Again by (1), this implies that $\mathbf{r k}_{w^{\prime}}\left(u^{\prime}\right)=n-1$. Hence, $w^{\prime}$ also has complete preferences and finds $M(w)$ acceptable, implying that $M(w)$ finds $w^{\prime}$ acceptable. Since $u \neq u^{\prime}$ and $\mathbf{r k}_{w}(u)=n-1$, we infer that $w^{\prime} \neq w$ because $\mathbf{r k}_{w^{\prime}}\left(u^{\prime}\right)=n-1$. This implies that $M(w)$ also finds at least two agents acceptable, namely $w$ and $w^{\prime}$. Since no two agents can have rank $n-1$ in the same preference list it must hold that the second-most preferred agents of all agents from $U$ are different from each other. Thus, using (1), each agent $w \in W$ must have complete preferences. By the symmetry of acceptability, each agent $u \in U$ must also have complete preferences.

We are now ready to prove that $P$ is position-wise distinct when $n \geq 3$. This is clear if each agent finds only one other agent acceptable. Otherwise, there exists at least one unmatched pair of agents. We will show the stronger statement that, for each unmatched pair $\{u, w\}$, with $u \in U$ and $w \in W$ and for each $z \in[n-1]$ it holds that

$$
\mathrm{rk}_{u}(w)=z \text { if and only if } \mathrm{rk}_{w}(u)=n-z .
$$

(Note that we can replace "if and only if" by "only if" to obtain an equivalent statement, but the former is more convenient when we prove it by induction below.) To see that (2) implies that $P$ is top-choice, suppose, for the sake of contradiction, that there are three distinct agents $x_{1}, x_{2}, y$ and an integer $z$ such that $\mathrm{rk}_{x_{1}}(y)=\mathrm{rk}_{x_{2}}(y)=z$. Since $M$ is top-choice, $z>0$. Clearly, $z \leq n-1$. Thus, by $(2) \mathrm{rk}_{y}\left(x_{1}\right)=\mathrm{rk}_{y}\left(x_{2}\right)=n-z$, a contradiction.

We show (2) via induction on the rank index $z:=\mathrm{rk}_{u}(w)$, starting with the base case $z=1$. To this end, let $\{u, w\}$ be an unmatched pair. To show the "only if" part of (2), assume that $z=\mathrm{rk}_{u}(w)=1$. By (1), it follows that $\mathrm{rk}_{w}(u) \geq n-1$. Since the rank of each agent is at most $n-1$, it follows that $\mathrm{rk}_{w}(u)=n-1=n-z$.

For the "if" part of the base case suppose, towards a contradiction, that there is an unmatched pair $\{u, w\}$ with $\mathrm{rk}_{w}(u)=n-1$ but $\mathbf{r k}_{u}(w) \neq 1$. By (1), it follows that $\mathbf{r k}_{u}(w)>1$. Since each agent has complete preferences, by the above reasoning, there exists an other agent $u^{\prime} \in U \backslash\{u\}$ with $\mathrm{rk}_{u^{\prime}}(w)=1$. However, then by the "if" part of the base case, it follows that $\mathrm{rk}_{w}\left(u^{\prime}\right)=n-1-\mathrm{a}$ contradiction. Thus, (2) holds when $z=1$.

For the induction assumption, assume that (2) holds for every index $z^{\prime} \leq z-1$. For the "only if" part, consider an unmatched pair $\{u, w\}$ with $\mathrm{rk}_{u}(w)=z$. By $(1)$, it follows that $\mathrm{rk}_{w}(u) \geq n-z$. Suppose, for the sake of contradiction, that $\mathrm{rk}_{w}(u)=n-z^{\prime}$ with $z^{\prime}<z$. By the "if" part of the induction assumption, we infer that $\mathrm{rk}_{u}(w)=z^{\prime}<z-$ a contradiction.

The "if" part of the induction step follows analogously.

Now we discuss the trade-offs formalized in the problems regarding near stability and social optimality. As mentioned in Example 1.2 even a single swap in the preference profile can improve 
the egalitarian cost of the stable matching by $\Omega\left(n^{2}\right)$. However, this is not the case when the social optimality is measured by the number of agents who will have a partner in the matching.

Theorem 2.6. Let $P_{1}$ and $P_{2}$ be two preference profiles with $\tau\left(P_{1}, P_{2}\right)=1$. Let $S_{1}$ and $S_{2}$ denote the set of agents that are unmatched by any stable matching of $P_{1}$ and of $P_{2}$ respectively. Then, $\left|\left(S_{1} \backslash S_{2}\right) \cup\left(S_{2} \backslash S_{1}\right)\right| \leq 2$.

Proof. Without loss of generality, assume that profile $P_{2}$ is obtained from $P_{1}$ by swapping agents $w_{1}$ and $w_{2}$ in the preference list of agent $u_{1}$ so that $u_{1}$ prefers $w_{1}$ to $w_{2}$ in $P_{1}$ and $w_{2}$ to $w_{1}$ in $P_{2}$.

By Proposition 2.3, in order to show the statement, it suffices to show that $P_{1}$ and $P_{2}$ admit stable matchings $M_{1}$ and $M_{2}$, respectively, such that the following is satisfied. Let $S_{1}$ and $S_{S}$ denote the set of agents that is unmatched under $M_{1}$ and $M_{2}$, respectively. Then, $\left|\left(S_{1} \backslash S_{2}\right) \cup\left(S_{2} \backslash S_{1}\right)\right| \geq 2$.

To achieve this, we start with an arbitrary but fixed stable matching, $M_{1}$, of $P_{1}$. And we will show how to modify $M_{1}$ to obtain a stable matching of $P_{2}$ such that the set of unmatched agents differ by at most two agents.

Observe that if $M_{1}$ would not be stable for profile $P_{2}$ anymore, then $\left\{u_{1}, w_{2}\right\}$ is the only possible blocking pair because $P_{1}$ and $P_{2}$ differ only by one single swap of the preference list of agent $u_{1}$. If $M_{1}$ is not stable for $P_{2}$, then we modify $M_{1}$ in the following way. We break the pairs $\left\{u_{1}, M_{1}\left(u_{1}\right)\right\}$ and $\left\{M_{1}\left(w_{2}\right), w_{2}\right\}$ (if they exist) and we replace them with $\left\{u_{1}, w_{2}\right\}$. Now, there are two new unmatched agents: $M_{1}\left(w_{2}\right) \in U$ and $M_{1}\left(u_{1}\right) \in W$. Further, if we remove these agents from the consideration, the matching would be stable.

We now proceed as follows. We will perform a sequence of changes to $M_{1}$. After each change we will keep in the penalty box at most two unmatched agents, one from $U$ and one from $W$, starting with $M_{1}\left(w_{2}\right)$ and $M_{1}\left(u_{1}\right)$. Further, each change will keep satisfying the following invariant: if we remove the agents contained in the penalty box, then the resulting matching would be stable. Let us now describe the way in which we perform the changes. Let $M$ be the matching at the current iteration. We take out an agent $u \in U$ from the penalty box (if such an agent does not exist, we stop). Agent $u$ might be involved in a number of blocking pairs - if it does not, we stop. We take $u$ 's most preferred agent $w \in W$ such that $\{u, w\}$ is a blocking pair of the current matching $M$-we remove $\{M(w), w\}$ from the matching $M$ and replace it with $\{u, w\}$. Finally, we add $M(w)$ to the penalty box. Clearly, $u$ cannot be involved in any blocking pair, thus, any blocking pair must involve an agent from the penalty box; hence the invariant is indeed satisfied.

Each such a change replaces a $U$-agent from the penalty box with another $U$-agent. Further each change improves one of the $W$-agents by giving her a more preferred partner. Thus, our procedure must stop at some point. When this is the case we remove the $U$-agent from the penalty box. Then, we perform an analogous procedure but each time replacing a $W$-agent in the penalty box with another $W$-agent. By an analogous arguments, such changes keep the invariant satisfied and the procedure finally stops.

Clearly, when the procedure stops, there are no blocking pairs. Further, the resulting matching has at most two more agents without partners than $M_{1}$.

Repeated application of Theorem 2.6 yields that, in order to increase the number of matched agents by $\ell \in \mathbb{N}$ in a given stable matching of some profile we have to allow for at least $\ell / 2$ swaps. In other words, if a stable matching leaves $s$ agents unmatched, then there is a globally $d$-nearly stable perfect matching only if $d \geq s / 2$.

Let us recall the notion of $r$-maximal stability for the case with linear orders [12]: A matching $M$ is $r$-maximally stable if for each unmatched pair $\{u, v\} \notin M$, it holds that $\min \left\{\mathrm{rk}_{u}(M(u))-\right.$ 
$\left.\mathrm{rk}_{u}(v), \mathrm{rk}_{v}(M(v))-\mathrm{rk}_{v}(u)\right\} \leq r$. At the first glance, this notion looks quite different from local $d$-near stability; we show below that in fact they are equivalent. Moreover, local $\mathrm{d}_{\mathrm{L}}$-near stability is equivalent to the following measure of the weight of a blocking pair. We say that a matching $M$ is $\mathrm{d}_{\mathrm{L}}$-nearly bp stable if for each blocking pair $b \in \mathcal{B P}(P, M)$, there exists a profile $P_{b}^{\prime}$ such that $b \notin \mathcal{B P}\left(P_{b}^{\prime}, M\right)$ and $\tau\left(P, P_{b}^{\prime}\right) \leq \mathrm{d}_{\mathrm{L}}$.

Proposition 2.7. Let $P$ be a preference profile without ties, $M$ be a matching for $P$, and $\mathrm{d}_{\mathrm{L}} a$

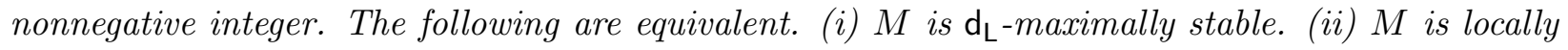

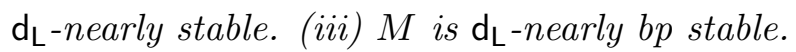

Proof. (i) $\Rightarrow$ (ii): Construct a directed graph $G$ on the set $V$ of agents as follows. For each blocking pair $\{u, v\} \in \mathcal{B P}(P, M)$ find the agent, say $u$, such that $\mathrm{rk}_{u}(M(u))-\mathrm{rk}_{u}(v) \leq \mathrm{rk}_{v}(M(v))-\mathrm{rk}_{v}(u)$ and add the $\operatorname{arc}(v, u)$ to $G$ (that is, add an arc directed towards $u$ ).

To obtain a modified profile $P^{\prime}$ in which $M$ is stable, define, for each agent $u$, a set of swaps in $u$ 's preference list as follows. Let $B_{u}$ be the set of agents $v$, such that $(v, u)$ is an arc in $G$. Pick $w=\operatorname{argmin}_{v \in B_{u}} \mathbf{r k}_{u}(v)$. Observe that $\mathbf{r k}_{u}(M(u))-\mathbf{r k}_{u}(w) \leq d$ since $(w, u)$ is an arc in $G$ and since $M$ is $\mathrm{d}_{\mathrm{L}}$-maximally stable. Swap $M(u)$ in $u$ 's preference list with the agent directly preceding $M(u)$ until $M(u) \succ_{u}^{P^{\prime}} w$ in the resulting profile $P^{\prime}$. In this way, for each agent we have made at most $\mathrm{d}_{\mathrm{L}}$ swaps to obtain $P^{\prime}$.

Note that throughout the swapping process no new blocking pairs are introduced, that is, $\mathcal{B P}\left(P^{\prime}, M\right) \subseteq \mathcal{B P}(P, M)$, because in each step only a matched agent improves her rank in its matched partner's preference list. Moreover, for each blocking pair $\{u, v\} \in \mathcal{B P}(P, M)$, we have either $M(u) \prec_{u}^{P^{\prime}} v$ or $M(v) \prec_{v}^{P^{\prime}} u$ by construction. Thus, $M$ is stable with respect to $P^{\prime}$, showing that $M$ is locally $\mathrm{d}_{\mathrm{L}}$-nearly stable.

(ii) $\Rightarrow$ (iii): Let $P^{\prime}$ be a profile as promised by local $d_{\mathrm{L}}$-near stability. For each blocking pair $\{u, v\} \in \mathcal{B P}(P, M)$ either $M(v)$ has been swapped before $u$ in $v$ 's preference list or $M(u)$ has been swapped before $v$ in $u$ 's preference list using in either case at most $d$ swaps. Restricting $P^{\prime}$ to only these swaps yields a profile $P_{\{u, v\}}^{\prime}$ as required by $\mathrm{d}_{\mathrm{L}}$-nearly bp stable.

(iii) $\Rightarrow$ (i): Let $\{u, v\}$ be an unmatched pair under $M$. If $\{u, v\} \notin \mathcal{B P}(P, M)$, then $\min \left\{\mathrm{rk}_{u}(M(u))-\mathrm{rk}_{u}(v), \mathrm{rk}_{v}(M(v))-\mathrm{rk}_{v}(u)\right\} \leq 0 \leq \mathrm{d}_{\mathrm{L}}$. Otherwise, $\{u, v\} \in \mathcal{B P}(P, M)$. By $\mathrm{d}_{\mathrm{L}}$-nearly bp stability, there are $\mathrm{d}_{\mathrm{L}}$ swaps such that in the resulting profile $P^{\prime}$ we have $M(u) \succ_{u}^{P^{\prime}} v$ or $M(v) \succ_{v}^{P^{\prime}} u$ because $\{u, v\} \notin \mathcal{B P}\left(P^{\prime}, M\right)$. Since $\{u, v\} \in \mathcal{B P}(P, M)$, meaning that both $v \succ_{u}^{P} M(u)$ and $u \succ_{v}^{P} M(v)$ hold, and since $\tau\left(P, P^{\prime}\right) \leq \mathrm{d}_{\mathrm{L}}$, we have $\mathrm{rk}_{u}(M(u))-\mathrm{rk}_{u}(v) \leq \mathrm{d}_{\mathrm{L}}$ or $\mathrm{rk}_{v}(M(v))-\mathrm{rk}_{v}(u) \leq \mathrm{d}_{\mathrm{L}}$. In other words, $M$ is $\mathrm{d}_{\mathrm{L}}$-maximally stable.

\section{A Polynomial-Time Algorithm for Finding Robust Matchings}

In this section we present a polynomial-time algorithm for the RoBUst MATCHing problem. First, in Section 3.1 we provide a brief overview of tools and results from the literature that we will use in our algorithm. We remark that all these results are originally stated for complete preferences. Nevertheless, since all stable matchings match the same set of agents (Proposition 2.3), we can verify that they also hold when the preferences may be incomplete. The results described in the subsequent sections, starting from Section 3.2, are our original contributions. 


\subsection{Preliminaries}

Recall that a pair $\{u, w\}$ with $u \in U$ and $w \in W$ is a stable pair of a preference profile $P$ if it is contained in at least one stable matching of $P$.

Proposition 3.1 ([18, Theorem 3.4.3]). For each preference profile, after $O\left(n^{4}\right)$ preprocessing time, determining whether a given set $Q$ of $t$ pairs is a stable set of $P$ can be done in $O\left(t^{2}\right)$ time.

As already observed in the literature, the set of all stable matchings for a given preference profile forms a lattice-a specific partially ordered set-that is useful in designing algorithms for finding special kinds of stable matchings. The maximum and minimum elements are so-called optimal stable matchings: Consider a preference profile $P$ with two sets, $U$ and $W$, of agents and consider two matchings $M$ and $M^{\prime}$. We say that an agent $a \in U \cup W$ prefers $M$ to $M^{\prime}$, denoted as $M \succ_{a} M^{\prime}$, if $\mathbf{r k}_{a}(M(a))<\mathrm{rk}_{a}\left(M^{\prime}(a)\right)$. Similarly, agent a weakly prefers $M$ to $M^{\prime}$, denoted as $M \succeq_{a} M^{\prime}$, if $M(a)=M^{\prime}(a)$ or $\mathrm{rk}_{a}(M(a))<\mathrm{rk}_{a}\left(M^{\prime}(a)\right)$. Accordingly, we say that $M$ is a $U$-optimal (resp. $W$-optimal) stable matching if it is stable and there is no other stable matching $M^{\prime}$ different from $M$ such that each agent from $U$ prefers $M^{\prime}$ to $M$.

It is well-known that $U$-optimal and $W$-optimal stable matchings are unique. The concepts of $U$-optimal and $W$-optimal stable matchings are already illustrated in Example 1.1.

Proposition 3.2 below shows that, when comparing two stable matchings, an improvement of an agent $u \in U$ always comes at the cost of some other agent from $W$.

Proposition 3.2 ([18, Theorem 1.3.1, Chapter 1.4.2]). Let $M_{1}$ and $M_{2}$ be two stable matchings of the same preference profile with (possibly) incomplete preferences, and let $u$ and $w$ be two agents such that $M_{1}(u)=w$ but $M_{2}(u) \neq w$. Then, $M_{1} \succ_{u} M_{2}$ if and only if $M_{2} \succ_{w} M_{1}$.

Finally, we recall that the famous Gale/Shapley algorithm always finds the $U$-optimal (or, depending on the variant of the algorithm used, the $W$-optimal) stable matching.

Proposition 3.3 ([15],[18, Chapter 1.4.2]). The U-optimal and the $W$-optimal stable matchings of a preference profile always exist and can be found in $O\left(n^{2}\right)$ time.

We now review a fundamental object, rotations, and some well-known structural properties of stable matchings. These concepts will play an instrumental role in our analysis in the subsequent sections. For more details, we refer to the exposition by Gusfield and Irving [18].

Definition 3.4 (Successor agent, rotations, and rotation elimination). Let $P$ be a preference profile with two disjoint sets of agents, $U$ and $W$, and with (possibly) incomplete preferences. Given a stable matching $M \in \mathcal{S M}(P)$, for each agent $u \in U$, we define its $\operatorname{successor}_{\operatorname{succ}}(u)$ as the first (after $M(u)$ ) agent $w$ on the preference list of $u$ such that $w$ is matched under $M$ and prefers $u$ to its partner $M(w)$. We illustrate the concept of the successor below:

$$
u: \ldots M(u) \ldots \operatorname{succ}_{M}(u) \ldots \quad \operatorname{succ}_{M}(u): \ldots u \ldots M\left(\operatorname{succ}_{M}(u)\right) \ldots
$$

A sequence $\rho=\left(\left(u_{0}, w_{0}\right),\left(u_{1}, w_{1}\right), \ldots,\left(u_{r-1}, w_{r-1}\right)\right)$ of pairs is called a rotation if there exists a stable matching $M \in \mathcal{S M}(P)$ such that for each $i \in\{0,1, \ldots, r-1\}$ we have $\left(u_{i}, w_{i}\right) \in U \times W$, $M\left(u_{i}\right)=w_{i}$, and $\operatorname{succ}_{M}\left(u_{i}\right)=w_{i+1}$ (index $i+1$ taken modulo $\left.r\right)$. We say rotation $\rho$ is exposed in $M$.

We use the notation $M / \rho$ to refer to the matching resulting from $M$ by replacing each pair $\left\{u_{i}, w_{i}\right\}$ with $\left\{u_{i}, w_{i+1}\right\}$. Formally,

$$
M / \rho=M \backslash\left\{\left\{u_{i}, w_{i}\right\} \mid 0 \leq i \leq r-1\right\} \cup\left\{\left\{u_{i}, w_{i+1}\right\} \mid 0 \leq i \leq r-1\right\} .
$$

The transformation of $M$ to $M / \rho$ is called the elimination of $\rho$ from $M$. 
Eliminating a rotation from a stable matching results in another stable matching [18]. The concepts from Definition 3.4 are illustrated in the example below.

Example 3.5. Consider the profile in Example 1.1. Relative to $M_{1}$, agent $w_{2}$ is the first agent among all agents in the preference list of $u_{1}$ that prefer $u_{1}$ to their respective partners. Thus, $\operatorname{succ}_{M_{1}}\left(u_{1}\right)=w_{2}$. Sequence $\left(\left(u_{1}, w_{2}\right),\left(u_{2}, w_{3}\right),\left(u_{3}, w_{4}\right),\left(u_{4}, w_{1}\right)\right)$ is the only rotation exposed in $M_{1}$.

Interestingly, while a given profile with $O(n)$ agents may admit exponentially $(O(n !))$ many different stable matchings, the number of rotations is polynomial $\left(O\left(n^{2}\right)\right)$ [18, Corollary 3.2.1]. Indeed, the set of all rotations gives a compact representation of the set of all possible stable matchings for a given preference profile. To determine robustness efficiently, we will use this representation intensely.

The next structural result concerns the properties of a stable matching after eliminating a rotation $\rho$.

Proposition 3.6 ([18, Theorem 2.5.6, Lemma 3.2.1, Lemma 3.2.2]). Consider a preference profile $P$ with two disjoint sets of agents, $U$ and $W$, and with (possibly) incomplete preferences. For each two agents $u \in U$ and $w \in W$, the following holds; recall that $x \succeq y$ means that $x=y$ or $x \succ y$.

(i) $\{u, w\}$ is in a stable matching if and only if either it is in the $W$-optimal stable matching or $(u, w)$ belongs to some rotation.

(ii) There is at most one rotation $\rho$ with $\rho=\left(\left(u_{0}, w_{0}\right), \ldots,\left(u_{r-1}, w_{r-1}\right)\right)$ such that for some $i \in$ $\{0, \ldots, r-1\}$ it holds that $u=u_{i}$ and $w_{i} \succeq_{u} w \succ_{u} w_{i+1}$.

(iii) There is at most one rotation $\rho$ with $\rho=\left(\left(u_{0}, w_{0}\right), \ldots,\left(u_{r-1}, w_{r-1}\right)\right)$ such that for some $i \in$ $\{0, \ldots, r-1\}$ it holds that $u=u_{i}$ and $w=w_{i+1}$.

(iv) There is at most one rotation $\rho$ with $\rho=\left(\left(u_{0}, w_{0}\right), \ldots,\left(u_{r-1}, w_{r-1}\right)\right)$ such that for some $i \in$ $\{0, \ldots, r-1\}$ it holds that $w=w_{i}$ and $u_{i-1} \succ_{w} u \succeq_{w} u_{i}$.

Now we are ready to introduce the notion of the rotation poset of a given preference profile $P$. As we will see later on, each stable matching can be obtained by performing a number of eliminations of rotations on the $U$-optimal stable matching. When starting from $U$ some rotations can be exposed only after some other have been already eliminated. This induces a partial order on rotations and defines the rotation poset.

Definition 3.7 (Predecessors of rotations, the rotation poset, and the rotation digraph). Let $\pi$ and $\rho$ be two rotations for a preference profile $P$. We say that $\pi$ is a predecessor of $\rho$, written as $\pi \triangleright^{P} \rho$, if no stable matching in which $\rho$ is exposed can be obtained from the $U$-optimal stable matching by a sequence of eliminations of rotations without eliminating $\pi$ first. The reflexive closure of the relation $\triangleright^{P}$, denoted as $\unrhd^{P}$, defines a partial order on the set of all rotations and is called the rotation poset for $P$. We abbreviate the name of a subset of the poset that is closed under predecessors as a closed subset.

An alternative representation of the rotation poset $\unrhd(P)$ is through an acyclic directed graph, called rotation digraph of $P$ and written as $G(P)$, whose vertex set is the set of rotations of $P$, and there is a direct arc from rotation $\pi$ to rotation $\rho$ if and only if $\pi$ precedes $\rho$ and there is no other rotation $\sigma$ such that $\pi \triangleright^{P} \sigma \triangleright^{P} \rho$.

The following example illustrates the rotation poset of profile given in Example 1.1. 
Example 3.8. Let us consider the profile $P$ given in Example 1.1 again. As we mentioned in Example 3.5, rotation $\pi_{1}=\left(\left(u_{1}, w_{2}\right),\left(u_{2}, w_{3}\right),\left(u_{3}, w_{4}\right),\left(u_{4}, w_{1}\right)\right)$ is the only rotation exposed in the $U$-optimal stable matching $M_{1}$. After eliminating $\pi_{1}$ from $M_{1}$, we obtain the stable matching $M_{3}=$ $M_{1} / \pi_{1}$. One can also verify that the sequence $\pi_{2}=\left(\left(u_{1}, w_{3}\right),\left(u_{3}, w_{1}\right)\right)$ and $\pi_{3}=\left(\left(u_{2}, w_{4}\right),\left(u_{4}, w_{2}\right)\right)$ are the only two rotations exposed in stable matching $M_{3}$. After eliminating $\pi_{2}$ from $M_{3}$, we obtain the stable matching $M_{4}=M_{3} / \pi_{2}$. After eliminating $\pi_{3}$ from $M_{3}$, we obtain the stable matching $M_{5}=M_{3} / \pi_{3}$. After eliminating rotation $\pi_{3}$ from $M_{4}$ or eliminating the rotation $\pi_{2}$ from $M_{5}$, we obtain the $W$-optimal stable matching $M_{2}$.

Since $\pi_{1}$ is only exposed in $M_{1}$ and since $\pi_{2}$ and $\pi_{3}$ are only exposed after the elimination of $\pi_{1}$ we have that $\pi_{2}$ and $\pi_{3}$ are two (direct) successors of $\pi_{1}$.

The Hasse diagram on the right-hand side depicts how the stable matchings for $P$ are related to each other, in terms of dominance with respect to the satisfaction of the agents from $U$. Herein, the matchings are depicted as matrices such that each pair in a matching is represented by a column in the corresponding matrix.

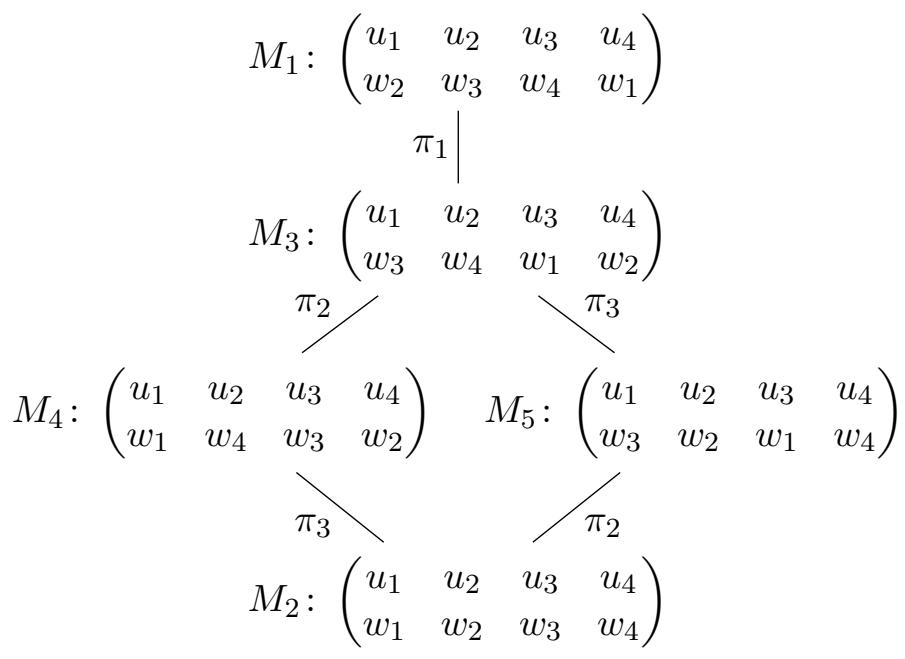

Finally, let us describe a central result from the literature that relates rotations and stable pairs.

Proposition 3.9 ([18, Theorem 2.5.7, Lemma 3.3.2]). Let $R$ denote the set of all rotations of $a$ preference profile $P$, and let $G(P)$ denote the rotation digraph of $P$.

(i) A matching $M$ is a stable matching of $P$ if and only if there is a closed subset of rotations $R^{\prime} \subseteq R$ with respect to the precedence relation $\triangleright^{P}$ such that $M$ can be generated by taking the $U$-optimal stable matching and by eliminating the rotations in $R^{\prime}$ in an order consistent with $\triangleright^{P}$.

(ii) The rotation set $R$ and the rotation digraph $G(P)$ can be computed in $O\left(n^{2}\right)$ time.

\subsection{Profile Characterization}

For a given profile $P$ with $O(n)$ agents and a given swap distance bound $d=O(n)$ there are exponentially many profiles which are within swap distance $d$ to $P$. In this section, we show that we do not need to consider all of them in order to find a $d$-robust matching. Instead, we characterize them based on pairs of shifts. Briefly put, a shift is a set of swaps which all involve swapping the same agent forward in a single preference list. We describe a polynomial-size family of "relevant" profiles and we characterize each of them through a pair of shifts - such a pair of shifts will be represented by a quadruple of agents. Intuitively, if there exists a profile $P^{\prime}$ witnessing that a certain matching $M$ is not $d$-robust, and if $P^{\prime}$ contains more than two shifts with respect to the original profile $P$, then $P^{\prime}$ can be represented by a number of profiles which satisfy the following. Each of these profiles contains only two shifts and one of them witnesses that $M$ is not $d$-robust. Later on, we will show that the 
quadruples which characterize the profiles relevant for checking $d$-robustness are closely related to certain rotations - this will give us the tools essential for constructing a polynomial-time algorithm.

Definition 3.10 (Stable quadruples and swap sets). Let $P=\left(\left(\succ_{u}^{P}\right)_{u \in U},\left(\succ_{w}^{P}\right)_{w \in W}\right)$ be a preference profile for the two agent sets $U$ and $W$. A stable quadruple (with respect to $P$ ) is a quadruple $\left(u^{*}, w^{*}, u, w\right)$ of four distinct agents with $u^{*}, u \in U$ and $w^{*}, w \in W$ such that there exists a stable matching for $P$ that contains both $\left\{u^{*}, w\right\}$ and $\left\{u, w^{*}\right\}$.

For each stable quadruple $q=\left(u^{*}, w^{*}, u, w\right)$ of $P$, we define the swap set associated with $P$ and $q$, denoted as $\mathcal{S H}(P, q)$, as the smallest set of swaps which involve the following two kinds of shifts in the preference lists of $u^{*}$ and $w^{*}$. 1. The first kind of shifts puts agent $w^{*}$ forward until she is right in front of $w$ in the preference list of $u^{*}$, and 2. the second kind of shifts puts agent $u^{*}$ forward until she is right in front of $u$ in the preference list of $w^{*}$. If $w^{*}$ (resp. $u^{*}$ ) is already in front of $w$ (resp. u), then no swap in the corresponding preference list is needed. Formally,

$$
\mathcal{S H}(P, q):=\bigcup_{y \in W: w \succ_{u^{*}}^{P} y \succ_{u^{*}}^{P} w^{*}}\left\{\left(u^{*},\left\{y, w^{*}\right\}\right)\right\} \cup \bigcup_{x \in U: u \succeq_{w^{*}}^{P} x \succ_{w^{*}}^{P} u^{*}}\left\{\left(w^{*},\left\{u^{*}, x\right\}\right)\right\} .
$$

Herein, the notation $x \succeq y$ means either $x=y$ or $x \succ y$. Further, let $\operatorname{shl}\left(\succ_{u^{*}}^{P}, q\right)$ denote the preference list resulting from starting with $\succ_{u^{*}}^{P}$ and performing the swaps from $\mathcal{S H}(P, q)$ that involve the preference list of $u^{*}$. Analogously, let $\operatorname{shl}\left(\succ_{w^{*}}^{P}, q\right)$ denote the preference list resulting from starting with $\succ_{w^{*}}^{P}$ and performing the swaps from $\mathcal{S H}(P, q)$ that involve the preference list $\succ_{w^{*}}^{P}$. Now, let $P[\operatorname{SH}(P, q)]$ denote the preference profile resulting from $P$ by replacing the preference lists of $u^{*}$ and $w^{*}$ with $\operatorname{shl}\left(\succ_{u^{*}}^{P}, q\right)$ and $\operatorname{shl}\left(\succ_{w^{*}}^{P}, q\right)$, respectively. Formally,

$$
P[\mathcal{S H}(P, q)]:=\left(\left(\succ_{x}^{P}\right)_{x \in U \backslash\left\{u^{*}\right\}}+\operatorname{shl}\left(\succ_{u^{*}}^{P}, q\right),\left(\succ_{y}^{P}\right)_{y \in W \backslash\left\{w^{*}\right\}}+\operatorname{shl}\left(\succ_{w^{*}}, q\right)\right) .
$$

Example 3.11. For an illustration, let us consider the profile given in Example 1.1, denoted as $P=\left(\left(\succ_{u_{i}}^{P}\right)_{u_{i} \in U},\left(\succ_{w_{i}}^{P}\right)_{w_{i} \in W}\right)$, and the following stable quadruple $q=\left(u_{3}, w_{2}, u_{4}, w_{1}\right)$; note that $\left\{\left\{u_{3}, w_{1}\right\},\left\{u_{4}, w_{2}\right\}\right\}$ is a stable set (see $\left.M_{3}\right)$. The swap set $\mathcal{S H}(P, q)$ consists of two swaps; both involve changing $u_{3}$ 's preference list: $\mathcal{S H}(P, q)=\left\{\left(u_{3},\left\{w_{2}, w_{3}\right\}\right),\left(u_{3},\left\{w_{2}, w_{1}\right\}\right)\right\}$.

By performing the swaps given in $\mathcal{S H}(P, q)$ on $\succ_{u_{3}}^{P}$ and on the preference profile, we obtain that $\operatorname{shl}\left(\succ_{u_{3}}^{P}, q\right)=\left\{u_{3}: w_{4} \succ w_{2} \succ w_{1} \succ w_{3}\right\}$, and $P[\mathcal{S H}(P, q)]=\left(\left(\succ_{u_{1}}^{P}, \succ_{u_{2}}^{P}, \succ_{u_{3}}^{P}, \operatorname{shl}\left(\succ_{u_{4}}^{P}, q\right)\right),\left(\succ_{w_{1}}^{P}, \succ_{w_{2}}^{P}\right.\right.$ $\left.\left., \succ_{w_{3}}^{P}, \succ_{w_{4}}^{P}\right)\right)$. Finally, we observe that in $P[\mathcal{S H}(P, q)], u_{4}$ prefers $w_{1}$ to $w_{2}$ and $w_{1}$ prefers $u_{4}$ to $u_{3}$.

A stable quadruple $q$ and the corresponding profile $P[\mathcal{S H}(P, q)]$ satisfy the following properties.

Observation 3.12. Let $P$ be a preference profile over the two agent sets $U$ and $W$, let $q$ be a stable quadruple with $q=\left(u^{*}, w^{*}, u, w\right)$ and let $Q=P[\mathcal{S H}(P, q)]$ denote the preference profile after performing the swaps in the set $\mathcal{S H}(P, q)$.

(i) Each agent $x \in U \cup W \backslash\left\{u^{*}, w^{*}\right\}$ other than $u^{*}$ and $w^{*}$ has $\succ_{x}^{Q}=\succ_{x}^{P}$.

(ii) If $w^{*} \succ_{u^{*}}^{P} w$, then $\succ_{u^{*}}^{Q}=\succ_{u^{*}}^{P}$; otherwise, for each two distinct agents $y, z \in W \backslash\left\{w^{*}\right\}$ the following holds. (a) $y \succ_{u^{*}}^{Q} z$ iff. $y \succ_{u^{*}}^{P} z$, (b) $y \succ_{u^{*}}^{Q} w^{*}$ iff. $y \succ_{u^{*}}^{P} w$, (c) $w^{*} \succ_{u^{*}}^{Q} y$ iff. $w \succeq_{u^{*}}^{P} y$.

(iii) If $u^{*} \succ_{w^{*}}^{P} u$, then $\succ_{w^{*}}^{Q}=\succ_{w^{*}}^{P}$; otherwise, for each two distinct agents $y, z \in U \backslash\left\{u^{*}\right\}$ the following holds. (a) $y \succ_{w^{*}}^{Q} z$ iff. $y \succ_{w^{*}}^{P} z$, (b) $y \succ_{w^{*}}^{Q} u^{*}$ iff. $y \succ_{w^{*}}^{P} u$, (c) $u^{*} \succ_{w^{*}}^{Q} y$ iff. $u \succeq_{w^{*}}^{P} y$.

(iv) In $P[\mathcal{S H}(P, q)]$, agent $u^{*}$ prefers $w^{*}$ to $w$, and agent $w^{*}$ prefers $u^{*}$ to $u$. 
Informally, we will argue that, to find a robust matching, it suffices to focus on profiles obtained by performing swaps induced by certain quadruples. Further, we will show that for each quadruple $q=\left(u^{*}, w^{*}, u, w\right)$ in profile $P[\mathcal{S H}(P, q)]$ we only need to ensure that $\left\{u^{*}, w^{*}\right\}$ is not a blocking pair.

Lemmas 3.13 and 3.14 below formalize our intuition that $\left\{u^{*}, w^{*}\right\}$ is the only possible blocking pair in $P[\mathcal{S H}(P, q)]$.

Lemma 3.13. Consider a preference profile $P$ and a stable matching $M$ for $P$. For a stable quadruple $q=\left(u^{*}, w^{*}, u, w\right)$, pair $\left\{u^{*}, w^{*}\right\}$ is the only possible blocking pair of $M$ in $P[\mathcal{S H}(P, q)]$.

Proof. Let $Q=P[\mathcal{S H}(P, q)]$. Suppose, towards a contradiction, that $M$ admits a blocking pair $\{x, y\}$ with $x \in U$ and $y \in W$ in profile $Q$ and that $\{x, y\} \neq\left\{u^{*}, w^{*}\right\}$. Since $Q$ differs from $P$ only in the preference lists of $u^{*}$ and $w^{*}$ and since $M$ is stable in $P$, it follows that either $x=u^{*}$ or $y=w^{*}$. If $x=u^{*}$, implying that $\left\{u^{*}, y\right\}$ is blocking $M$ in $Q$, then it holds that $y \succ_{u^{*}}^{Q} M\left(u^{*}\right)$ and $u^{*} \succ_{y}^{Q} M(y)$. However, since $\{x, y\} \neq\left\{u^{*}, w^{*}\right\}$ it follows that $y \neq w^{*}$, and that $y \succ_{u^{*}}^{P} M\left(u^{*}\right)$ (see Observation 3.12(ii)) and $u^{*} \succ_{y}^{P} M(y)$ (see Observation 3.12(i)). This implies that $\left\{u^{*}, y\right\}$ is also blocking $M$ in $P$, a contradiction to $M$ being stable in $P$. Analogously, we can derive a contradiction for the case of $x \neq u^{*}$ and $y=w^{*}$.

Lemma 3.14. Let $P_{1}$ and $P_{2}$ be two preference profiles for the same two disjoints sets $U$ and $W$, and let $M \in \mathcal{S M}\left(P_{1}\right)$ be a stable matching of $P_{1}$. Let $\left\{u^{*}, w^{*}\right\} \in \mathcal{B P}\left(P_{2}, M\right)$ be a blocking pair for $P_{2}$ with $u^{*} \in U$ and $w^{*} \in W$. Define $q=\left(u^{*}, w^{*}, M\left(w^{*}\right), M\left(u^{*}\right)\right)$. The following holds. (i) $\mathcal{B P}\left(P\left[\mathcal{S H}\left(P_{1}, q\right)\right], M\right)=\left\{\left\{u^{*}, w^{*}\right\}\right\}$. (ii) $\left|\mathcal{S H}\left(P_{1}, q\right)\right| \leq \tau\left(P_{1}, P_{2}\right)$.

Proof. To show the first statement, assume that $M$ is not stable in $P_{2}$ and let $\left\{u^{*}, w^{*}\right\}$ be a blocking pair of $M$ for $P_{2}$. Set $Q=P\left[\mathcal{S H}\left(P_{1}, q\right)\right]$.

By Observation 3.12(iv), we immediately get that $\left\{u^{*}, w^{*}\right\}$ is blocking $M$ in profile $Q$. The fact that $\left\{u^{*}, w^{*}\right\}$ is the only blocking pair follows from Lemma 3.13.

Now let us consider the second statement. By the definition of swap sets on $q$, we have:

$\left|\mathcal{S H}\left(P_{1}, q\right)\right|=\max \left(\mathrm{rk}_{u^{*}}\left(w^{*}, \succ_{u^{*}}^{P_{1}}\right)-\mathrm{rk}_{u^{*}}\left(M\left(u^{*}\right), \succ_{u^{*}}^{P_{1}}\right), 0\right)+\max \left(\mathrm{rk}_{w^{*}}\left(u^{*}, \succ_{w^{*}}^{P_{1}}\right)-\mathrm{rk}_{w^{*}}\left(M\left(w^{*}\right), \succ_{w^{*}}^{P_{1}}\right), 0\right)$.

Since $\left\{u^{*}, w^{*}\right\}$ is blocking $M$ in $P_{2}$ but $M$ is stable for $P_{1}$, it holds that

$$
w^{*} \succ_{u^{*}}^{P_{2}} M\left(u^{*}\right) \text { and } u^{*} \succ_{w^{*}}^{P_{2}} M\left(w^{*}\right) \text {, while } M\left(u^{*}\right) \succ_{u^{*}}^{P_{1}} w^{*} \text { or } M\left(w^{*}\right) \succ_{w^{*}}^{P_{1}} u^{*} .
$$

Thus,

$$
\begin{aligned}
\tau\left(P_{1}, P_{2}\right) \geq & \max \left(\mathrm{rk}_{u^{*}}\left(w^{*}, \succ_{u^{*}}^{P_{1}}\right)-\mathrm{rk}_{u^{*}}\left(M\left(u^{*}\right), \succ_{u^{*}}^{P_{1}}\right), 0\right)+ \\
& +\max \left(\mathrm{rk}_{w^{*}}\left(u^{*}, \succ_{w^{*}}^{P_{1}}\right)-\mathrm{rk}_{w^{*}}\left(M\left(w^{*}\right), \succ_{w^{*}}^{P_{1}}\right), 0\right) \\
= & \left|\mathcal{S H}\left(P_{1}, q\right)\right| .
\end{aligned}
$$

proving the statement.

Finally, the following lemma summarizes the informal intuition that we provided so far-it shows that when searching for a $d$-robust matching, we only need to focus on some relevant profiles which are close to the initial one. 
Lemma 3.15. Let $P_{0}$ be a preference profile for two disjoint sets of agents, $U$ and $W$, and let $d \in \mathbb{N}$ be a non-negative integer. A matching $M$ is d-robust for profile $P_{0}$ if and only if for each stable quadruple $q=\left(u^{*}, w^{*}, u, w\right)$ of $P_{0}$ such that $\left|\mathcal{S H}\left(P_{0}, q\right)\right| \leq d$, matching $M$ is also stable in $P\left[\mathcal{S H}\left(P_{0}, q\right)\right]$.

Proof. The "only if" direction is straight-forward because $M$ is stable in each profile $P$ with $\tau\left(P_{0}, P\right) \leq d$ and for each stable quadruple $q=\left(u^{*}, w^{*}, u, w\right)$ such that $\left|\mathcal{S H}\left(P_{0}, q\right)\right| \leq d$, it holds that $\tau\left(P_{0}, P\left[\mathcal{S H}\left(P_{0}, q\right)\right]\right)=\left|\mathcal{S H}\left(P_{0}, q\right)\right| \leq d$.

For the "if" direction, assume that there is a matching, called $M$, such that for each stable quadruple $q=\left(u^{*}, w^{*}, u, w\right)$ with $\left|\mathcal{S H}\left(P_{0}, q\right)\right| \leq d$, matching $M$ is stable in $P\left[\mathcal{S H}\left(P_{0}, q\right)\right]$. Suppose, for the sake of contradiction, that there is a preference profile $P$ with $\tau\left(P_{0}, P\right) \leq d$ such that $M$ is not stable in $P$. Let $\{x, y\}$ be a blocking pair of $M$ in $P$ with $x \in U$ and $y \in W$. Now let us consider the quadruple $q^{\prime}=(x, y, M(y), M(x))$. Note that $q^{\prime}$ is a stable quadruple with respect to $P_{0}$ since $M$ is stable for $P_{0}$. Since $\{x, y\} \in \mathcal{B P}(P, M)$, by Lemma 3.14(i), it follows that $\mathcal{B P}\left(P\left[\mathcal{S H}\left(P_{0}, q\right)\right], M\right)=\{\{x, y\}\}$ and, by Lemma 3.14(ii), $\left|\mathcal{S H}\left(P_{0}, q\right)\right| \leq \tau\left(P_{0}, P\right) \leq d-$ a contradiction to our assumption.

\subsection{Relation Between Stable Quadruples and Rotations}

Before we state our central results, we need one more element: In this subsection we define two specific rotations corresponding to a stable quadruple and we investigate their properties pertaining to robustness. The results stated in this subsection might look quite technical, yet we deliberately chose these particular formulations as we believe they make the analysis of our algorithm transparent.

Definition $3.16(\pi(q)$ and $\rho(q)$ for a stable quadruple $q)$. Let $P_{0}=\left(\succ_{x}^{P_{0}}\right)_{x \in U \cup W}$ be a preference profile with two sets of agents, $U$ and $W$, and consider a stable quadruple $q=\left(u^{*}, w^{*}, u, w\right)$.

We use the notation $\pi(q)$ to refer to a rotation $\pi:=\left(\left(u_{0}^{\prime}, w_{0}^{\prime}\right), \ldots,\left(u_{r-1}^{\prime}, w_{r-1}^{\prime}\right)\right)$ with $u^{*}=u_{i}^{\prime}$ (for some $i \in\{0, \ldots, r-1\}$ ) that fulfills the following conditions. (i) If $w^{*} \succ_{u^{*}}^{P_{0}} w$, then $w^{*}=w_{i}^{\prime}$ or $w_{i}^{\prime} \succ_{u^{*}}^{P_{0}} w^{*} \succ_{u^{*}}^{P_{0}} w_{i+1}^{\prime}$; (ii) Otherwise, meaning that $w \succ_{u^{*}}^{P_{0}} w^{*}$, then $w=w_{i+1}^{\prime}$.

We use the notation $\rho(q)$ to refer to a rotation $\rho:=\left(\left(u_{0}^{\prime}, w_{0}^{\prime}\right), \ldots,\left(u_{r-1}^{\prime}, w_{r-1}^{\prime}\right)\right)$ with $w^{*}=w_{i}^{\prime}$ (for some $i \in\{0, \ldots, r-1\}$ ) that fulfills the following conditions. (i) If $u^{*} \succ_{w^{*}}^{P_{0}} u$, then $u^{*}=u_{i-1}^{\prime}$ or $u_{i-1}^{\prime} \succ_{w^{*}}^{P_{0}} u^{*} \succ_{w^{*}}^{P_{0}} u_{i}^{\prime}$; (ii) Otherwise, meaning that $u \succ_{w^{*}}^{P_{0}} u^{*}$, then $u=u_{i}^{\prime}$.

The below figure illustrates the two specific rotations; recall that for two agents $x$ and $y$, the expression " $x \succeq y$ " means that $x=y$ or $x \succ y$.

Case (i): $\boldsymbol{u}^{*}$ prefers $\boldsymbol{w}^{*}$ to $\boldsymbol{w}$, i.e. $\boldsymbol{w}^{*} \succ_{\boldsymbol{u}^{*}}^{\boldsymbol{P}_{\mathbf{0}}} \boldsymbol{w}$. Then, $u^{*}: \underbrace{\prime}_{i} \succeq w^{*} \succ w_{i+1}^{\prime}$.

Case (ii): $\boldsymbol{u}^{*}$ prefers $\boldsymbol{w}$ to $\boldsymbol{w}^{*}$, i.e. $\boldsymbol{w} \succ_{\boldsymbol{u}^{*}}^{\boldsymbol{P}_{0}} \boldsymbol{w}^{*}$. Then, $u^{*}: w_{i}^{\prime} \succ w=w_{i+1}^{\prime} \succ w^{*}$.$$
\iota_{\pi(q)} \uparrow
$$

Case (i): $\boldsymbol{w}^{*}$ prefers $\boldsymbol{u}^{*}$ to $\boldsymbol{u}$, i.e. $\boldsymbol{u}^{*} \succ_{\boldsymbol{w}^{*}}^{\boldsymbol{P}_{\mathbf{0}}} \boldsymbol{u}$. Then, $w^{*}: \underbrace{\prime}_{u_{i-1}^{\prime} \succeq u^{*} \succ u_{i}^{\prime}}$. Case (ii): $\boldsymbol{w}^{*}$ prefers $\boldsymbol{u}$ to $\boldsymbol{u}^{*}$, i.e. $\boldsymbol{u} \succ_{\boldsymbol{w}^{*}}^{\boldsymbol{P}_{\mathbf{0}}} \boldsymbol{u}^{*}$. Then, $w^{*}: u_{i-1}^{\prime} \succ u=u_{i}^{\prime} \succ u^{*}$. 
Rotations $\pi(q)$ and $\rho(q)$ can be informally described as follows. Consider the preference profile $Q=P\left[\mathcal{S H}\left(P_{0}, q\right)\right]$. Rotation $\pi(q)$ is the first rotation (according to the precedence relation on rotations) that moves the partner of $u^{*}$ from $w^{*}$ or from an agent who is more preferred than $w^{*}$ to an agent that is less preferred than $w^{*}$, where the preference relation is according to profile $Q$. Similarly, rotation $\rho(q)$ is the first rotation that moves the partner of $w^{*}$ from an agent who is less preferred than $u^{*}$ to $u^{*}$ or to an agent that is more preferred than $u^{*}$, where the preference relation is according to profile $Q$. However, in Definition 3.16 we deliberately do not refer to profile $Q$ and define the rotations $\pi(q)$ and $\rho(q)$ solely based on $P_{0}$ in order to make the subsequent formal analysis and the algorithm as clear as possible.

Roughly speaking, eliminating rotation $\pi(q)$ could make a stable matching of the original profile not stable anymore in the new profile $Q$-indeed, this is the "first" rotation, elimination of which causes $u^{*}$ to prefer $w^{*}$ over its matched partner. In order to make sure that $\left\{u^{*}, w^{*}\right\}$ is not blocking the constructed matching in $Q$, we need to enforce that, whenever the matching includes $\pi(q)$, agent $w^{*}$ must obtain a partner who she prefers over $u^{*}$. This is achieved by also including $\rho(q)$ to the matching. In other words, when selecting rotations which should form a robust matching, adding $\rho(q)$ fixes some potential issues that arise as a result of adding $\pi(q)$ to the matching. This intuition is formalized in the subsequent lemmas and theorems. While the main idea is intuitive, the formal analysis is complex since we need to take care of a few technical nuances.

Note that, by our definition, neither $\pi(q)$ nor $\rho(q)$ needs to exist. However, if they exist, then they are unique. We will prove this statement in Lemma 3.18, below. The following example that illustrates the definitions of $\pi(q)$ and $\rho(q)$.

Example 3.17. Recall that in Example 3.8 we have derived the rotation poset of the profile given in Example 1.1, and $q=\left(u_{3}, w_{2}, u_{4}, w_{1}\right)$ is the stable quadruple discussed in Example 3.11.

Since $u_{3}$ prefers $w_{1}$ to $w_{2}$, to define $\pi(q)$, we are searching for a rotation, which includes $\left(u_{3}, x\right)$ for some agent $x \in W$ such that after the elimination of this rotation, $u_{3}$ receives agent $w_{1}$ as a partner. Rotation $\pi_{1}$ is the only rotation that fulfills this condition. Thus, $\pi(q):=\pi_{1}$. Let $P^{\prime}$ be the profile resulting from performing the two swaps given in $\mathcal{S H}(P, q)$. One can verify that in $P^{\prime}$ agent $u_{3}$ prefers $w_{2}$ to $w_{1}$. Thus, in the same profile, either (i) $u_{3}$ prefers $w_{2}$ to the partner assigned by a stable matching whose corresponding closed subset of rotations includes $\pi(q)$, or (ii) the partner of $u_{3}$ is $w^{*}=w_{2}$. Indeed, each rotation eliminated, for which rotation $\pi(q)$ is a predecessor, either makes $w_{2}$ still the partner of $u_{3}$ or changes the partner of $u_{3}$ to one which is less preferred than $w_{1}$ in the initial profile, including agent $w^{*}$ which is more preferred than $w_{1}$ in $P^{\prime}$. This means that such kind of stable matching may be blocked by $\left\{u_{3}, w_{2}\right\}$ in $P^{\prime}$.

As for $\rho(q)$, since $w_{2}$ prefers $u_{3}$ to $u_{4}$, we are searching for a rotation which includes $\left(y, w_{2}\right)$ such that

either $y=u_{3}$,

or $w_{2}$ prefers $u_{3}$ over $y$ and will obtain a partner which $w_{2}$ prefers over $y$.

Indeed, since $\pi_{3}$ satisfies (4), one can verify that $\rho(q)=\pi_{3}$. As a final remark, note that, since $\pi_{3}$ satisfies (4), by Gusfield and Irving [18, Lemma 2.5.1] it follows that no stable matching matches $u_{3}$ to $w_{2}$.

Observe that, after the elimination of this rotation, $w_{2}$ obtains $u_{2}$, which is her most preferred agent, i.e., an agent that is strictly better than $u_{3}$. Again, one can verify that in $P^{\prime}$ agent $w_{2}$ still prefers $u_{2}$ to $u_{3}$. Thus, $w_{2}$ prefers its partner, assigned by a stable matching whose corresponding 
closed subset includes $\rho(q)$, to $u_{3}$. However, this means that such kind of stable matching cannot be blocked by $\left\{u_{3}, w_{2}\right\}$.

For a comparison, let us consider another stable quadruple $q^{\prime}=\left(u_{1}, w_{2}, u_{4}, w_{1}\right)$. Since $u_{1}$ prefers $w_{2}$ to $w_{1}$ we are searching for a rotation which includes $\left(u_{1}, x\right)$ such that

either $x=w_{2}$,

or $u_{1}$ prefers $x$ over $w_{2}$ and will obtain a partner which $u_{1}$ prefers over $x$.

Since $\pi_{1}$ includes $\left(u_{1}, w_{2}\right)$, satisfying (5), we have that $\pi\left(q^{\prime}\right)=\pi_{1}$.

As for $\rho\left(q^{\prime}\right)$, since $w_{2}$ prefers $u_{4}$ to $u_{1}$, we need to find a rotation which includes $\left(u_{4}, w_{2}\right)$. Since $\pi_{3}$ includes $\left(u_{4}, w_{2}\right)$, we have that $\rho\left(q^{\prime}\right)=\pi_{3}$.

Rotations $\pi(q)$ and $\rho(q)$ are critical concepts that will be used by our algorithm for finding robust matchings. The next two lemmas provide tools which allow us to use these concepts conveniently. We start by showing that $\pi(q)$ and $\rho(q)$ are unique.

Lemma 3.18. Let $q=\left(u^{*}, w^{*}, u, w\right)$ be a stable quadruple of a preference profile $P$. The following holds.

1. If $\pi(q)$ exists, then it is unique.

2. If $\rho(q)$ exists, then it is unique.

Proof. For the first statement, assume that rotation $\pi(q)$ exists with $\pi(q)=$ $\left(\left(u_{0}^{\prime}, w_{0}^{\prime}\right), \ldots,\left(u_{r-1}^{\prime}, w_{r-1}^{\prime}\right)\right)$ and $u^{*}=u_{i}^{\prime}$. We distinguish between two cases.

Case (i): $w^{*} \succ_{u^{*}} w$. Thus, $w^{*}=w_{i}^{\prime}$ or $w_{i}^{\prime} \succ_{u^{*}}^{P} w^{*} \succ_{u^{*}}^{P} w_{i+1}^{\prime}$ by definition of $\pi(q)$. In either case, Proposition 3.6(ii) guarantees that $\pi(q)$ is unique.

Case (ii): $w \succ_{u^{*}} w^{*}$. By the definition of $\pi(q)$, we have that $w=w_{i+1}^{\prime}$. By Proposition 3.6 (iii), rotation $\pi(q)$ is unique.

Now, we turn to the second statement. Assume that rotation $\rho(q)$ exists with $\rho(q)=$ $\left(\left(u_{0}^{\prime}, w_{0}^{\prime}\right), \ldots,\left(u_{r-1}^{\prime}, w_{r-1}^{\prime}\right)\right)$ and $w^{*}=w_{i}^{\prime}$. Again, we distinguish between two cases:

Case (i): $u^{*} \succ_{w^{*}} u$. This implies that $u^{*}=u_{i-1}^{\prime}$ or $u_{i-1}^{\prime} \succ_{w^{*}}^{P} u^{*} \succ_{w^{*}}^{P} u_{i}^{\prime}$ by definition of $\rho(q)$. If $u^{*}=u_{i-1}^{\prime}$, then the uniqueness is guaranteed by Proposition 3.6(iii). If $u_{i-1}^{\prime} \succ_{w^{*}}^{P} u^{*} \succ_{w^{*}}^{P} u_{i}^{\prime}$, then the uniqueness follows from Proposition 3.6(iv).

Case (ii): $u \succ_{w^{*}} u^{*}$. By the definition of $\rho(q)$, we have that $u=u_{i}^{\prime}$. By Proposition 3.6 (iv), rotation $\rho(q)$ is unique.

The following result is a centerpiece of the algorithm, specifying exactly which constraints need to be fulfilled by a closed subset of rotations which corresponds to a robust matching.

Lemma 3.19. Let $P_{0}$ be a profile and $q=\left(u^{*}, w^{*}, u, w\right)$ be a stable quadruple of $P_{0}$. Let $Q=$ $P\left[\mathcal{S H}\left(P_{0}, q\right)\right]$ denote the profile after we perform the swaps in $\mathcal{S H}\left(P_{0}, q\right)$ on $P_{0}$. The following holds.

(i) Assume that $\pi(q)$ does not exist. If $w^{*} \succ_{u^{*}}^{P_{0}} w$, then each stable matching $N \in \mathcal{S M}\left(P_{0}\right)$ has $w^{*} \succ_{u^{*}}^{Q} N\left(u^{*}\right)$. If $w \succ_{u^{*}}^{P_{0}} w^{*}$, then each $N \in \mathcal{S M}\left(P_{0}\right)$ has either $N\left(u^{*}\right)=w^{*}$ or $w^{*} \succ_{u^{*}}^{Q} N\left(u^{*}\right)$.

(ii) Assume that $\rho(q)$ does not exist. If $u^{*} \succ_{w^{*}}^{P_{0}} u$, then each stable matching $N \in \mathcal{S M}\left(P_{0}\right)$ has $u^{*} \succ_{w^{*}}^{Q} N\left(w^{*}\right)$. If $u \succ_{w^{*}}^{P_{0}} u^{*}$, then each $N \in \mathcal{S M}\left(P_{0}\right)$ has either $N\left(u^{*}\right)=w^{*}$ or $u^{*} \succ_{w^{*}}^{Q} N\left(w^{*}\right)$.

(iii) If neither $\pi(q)$ nor $\rho(q)$ exist, then $\mathcal{S M}\left(P_{0}\right) \cap \mathcal{S M}(Q)=\emptyset$.

Let $S$ be a closed subset of rotations for $P_{0}$ and let $M$ be the corresponding stable matching. 
(iv) If $\pi(q)$ does not exist and $\rho(q)$ exists but $\rho(q) \notin S$, then $M \notin \mathcal{S M}(Q)$.

(v) If $\rho(q)$ exists and $\rho(q) \in S$, then $M \in \mathcal{S M}(Q)$.

(vi) If $\pi(q)$ exists and $\pi(q) \notin S$, then $M \in \mathcal{S M}(Q)$.

(vii) If $\pi(q)$ exists and $\pi(q) \in S$ and either $\rho(q)$ does not exist or $\rho(q)$ exists but $\rho(q) \notin S$, then $M \notin \mathcal{S M}(Q)$.

Proof. Statement (i). Assume that $\pi(q)$ does not exist. Since $q$ is a stable quadruple, by definition, $\left\{\left\{u^{*}, w\right\},\left\{u, w^{*}\right\}\right\}$ is a stable set. First, let us consider the case when $w^{*} \succ_{u^{*}}^{P_{0}} w$. Since $\pi(q)$ does not exist, by definition, $\left(u^{*}, w^{*}\right)$ is not in any rotation. Neither is $\left\{u^{*}, w^{*}\right\}$ in the $W$-optimal stable matching of $P_{0}$ because of the following. Since $w^{*} \succ_{u^{*}}^{P_{0}} w$ hold and $\{u, w\}$ is in some stable matching, say $M$, of $P_{0}$, it follows that $M\left(w^{*}\right) \succ_{w^{*}}^{P_{0}} u^{*}$, implying that $\left\{u^{*}, w^{*}\right\}$ is not in the $W$ optimal stable matching. Hence, by Proposition 3.6(i), $\left\{u^{*}, w^{*}\right\}$ is not in any stable matching of $P_{0}$. Thus, we only need to show that no stable matching $N \in \mathcal{S M}\left(P_{0}\right)$ has $N\left(u^{*}\right) \succ_{u^{*}}^{Q} w^{*}$. Towards a contradiction, suppose that there exists such a stable matching $N$ with $N\left(u^{*}\right) \succ_{u^{*}}^{Q} w^{*}$. By Observation 3.12(ii) and since $w^{*} \succ_{u^{*}}^{P_{0}} w$, it follows that $\succ_{u^{*}}^{P_{0}}=\succ_{u^{*}}^{Q}$, and so $N\left(u^{*}\right) \succ_{u^{*}}^{P_{0}} w^{*}$, implying that $N\left(u^{*}\right) \succ_{u^{*}}^{P_{0}} w^{*} \succ_{u^{*}}^{P_{0}} w$ because $w^{*} \succ_{u^{*}}^{P_{0}} w$. By the definition of stable quadruples, $\left\{u^{*}, w\right\}$ is in some stable matching. Thus, there are two stable matchings, where $u^{*}$ obtains a partner (namely, $N\left(u^{*}\right)$ ) who is more preferred than $w^{*}$, and a partner (namely, $w$ ) who is less preferred than $w^{*}$. By Proposition 3.6(i), this is a contradiction to the assumption that $\pi(q)$ does not exist.

Now, consider the case when $w \succ_{u^{*}}^{P_{0}} w^{*}$. Since $\left\{\left\{u^{*}, w\right\}\right\}$ is a stable set and $\pi(q)$ does not exist, we infer that $\left\{u^{*}, w\right\}$ is in the $U$-optimal stable matching, i.e., that every stable matching $N \in$ $\mathcal{S M}\left(P_{0}\right)$ has either $N\left(u^{*}\right)=w$ or $w \succeq_{u^{*}}^{P_{0}} N\left(u^{*}\right)$. By Observation 3.12(ii)(c), it follows that every stable matching $N \in \mathcal{S M}\left(P_{0}\right)$ has either $N\left(u^{*}\right)=w^{*}$ or $w^{*} \succ_{u^{*}}^{Q} N\left(u^{*}\right)$.

Statement (ii). Assume that $\rho(q)$ does not exist. Again, we consider two cases, starting with $u^{*} \succ_{w^{*}}^{P_{0}} u$. Since $\rho(q)$ does not exist, we can infer that $\left\{u^{*}, w^{*}\right\}$ does not belong to any stable matching of $P_{0}$. Indeed, if a stable matching containing $\left\{u^{*}, w^{*}\right\}$ existed, then there would be a rotation that changes the partner of $w^{*}$ from one which is less preferred than $u^{*}$ to $u^{*}$ (here, again we use the fact that $q$ is a quadruple and so $w^{*}$ is matched to $u$ in some stable matching), with respect to profile $P_{0}$.

Thus, we only need to show that no stable matching $N \in \mathcal{S M}\left(P_{0}\right)$ has $N\left(w^{*}\right) \succ_{w^{*}}^{Q} u^{*}$. Towards a contradiction, suppose that there exists such a stable matching $N$ with $N\left(w^{*}\right) \succ_{w^{*}}^{Q} u^{*}$. By Observation 3.12(iii) and since $u^{*} \succ_{w^{*}}^{P_{0}} w$, it follows that $\succ_{w^{*}}^{P_{0}}=\succ_{w^{*}}^{Q}$ and so that $N\left(w^{*}\right) \succ_{w^{*}}^{P_{0}} u^{*}$, implying that $N\left(w^{*}\right) \succ_{w^{*}}^{P_{0}} u^{*} \succ_{w^{*}}^{P_{0}} u$ because $u^{*} \succ_{w^{*}}^{P_{0}} u$. By the definition of stable quadruples, $\left\{u, w^{*}\right\}$ belongs to some stable matching. Summarizing, there exist two stable matchings where $w^{*}$ is matched to a partner which is less preferred than $u^{*}$ and a partner which is more preferred than $u^{*}$, respectively. However, this is a contradiction to the assumption that $\pi(q)$ does not exist.

Now, let us move to the case when $u \succ_{w^{*}}^{P_{0}} u^{*}$. Recall that, since $q$ is a stable quadruple, we know that $\left\{u, w^{*}\right\}$ belongs to some stable matching. Since $\rho(q)$ does not exist by Proposition 3.6(i) we infer that $\left\{u, w^{*}\right\}$ is in the $W$-optimal stable matching. In other words, for every stable matching $N \in \mathcal{S M}\left(P_{0}\right)$ we have either $u=N\left(w^{*}\right)$ or $u \succ_{w^{*}}^{P_{0}} N\left(w^{*}\right)$. By Observation 3.12(iii)(c), it follows that every stable matching $N \in \mathcal{S M}\left(P_{0}\right)$ has either $N\left(w^{*}\right)=u^{*}$ or $u^{*} \succ_{w^{*}}^{Q} N\left(w^{*}\right)$.

Statement (iii). Assume that neither $\pi(q)$ nor $\rho(q)$ exists. We distinguish between two cases.

Case (1): $w \succ_{u^{*}}^{P_{0}} w^{*}$. Since $\pi(q)$ does not exist, by statement (i), it follows that every stable 
matching $N \in \mathcal{S M}\left(P_{0}\right)$ has either $N\left(u^{*}\right)=w^{*}$ or $w^{*} \succ_{u^{*}}^{Q} N\left(u^{*}\right)$. Consider an arbitrary stable matching $N \in \mathcal{S M}\left(P_{0}\right)$, and first let us analyze the case when $N\left(u^{*}\right)=w^{*}$. Since $q$ is a stable quadruple, there exists a matching, call it $M^{\prime}$, such that $M^{\prime}\left(u^{*}\right)=w$ and $M^{\prime}(u)=w^{*}$. By our assumption, $u^{*}$ prefers $M^{\prime}$ to $N$; thus, by Proposition 3.2 we get that $w^{*}$ must prefer $N$ to $M^{\prime}$, i.e., it must hold that $u^{*} \succ_{w^{*}}^{P_{0}} u$. By statement (ii), that " $u^{*} \succ_{w^{*}}^{P_{0}} u$ " and the assumption that "rotation $\rho(q)$ does not exist" imply that $u^{*} \succ_{w^{*}}^{Q} N\left(w^{*}\right)$. This contradicts our assumption that $N\left(u^{*}\right)=w^{*}$. Now, let us move to the second alternative, when $w^{*} \succ_{u^{*}}^{Q} N\left(u^{*}\right)$. We know that $\rho(q)$ does not exist. Thus, by statement (ii), we get that $u^{*} \succ_{w^{*}}^{Q} N\left(w^{*}\right)$ because of the following.

- Either $u^{*} \succ_{w^{*}}^{P_{0}} u$, whence by statement (ii) we have $u^{*} \succ_{w^{*}}^{Q} N\left(w^{*}\right)$,

- or $u \succeq_{w^{*}}^{P_{0}} u^{*}$ and by statement (ii) we have that $u^{*} \succ_{w^{*}}^{Q} N\left(w^{*}\right)$ or that $N\left(u^{*}\right)=w^{*}$ (the latter case has just been handled; either way, we have that $u^{*} \succ_{w^{*}}^{Q} N\left(w^{*}\right)$.

Yet, this implies that $\left\{u^{*}, w^{*}\right\}$ is a blocking pair of $N$. Thus, $N \notin \mathcal{S M}(Q)$.

Case (2): $w^{*} \succ_{u^{*}}^{P_{0}} w$. Since $\pi(q)$ does not exist, from statement (i) it follows that every stable matching $N \in \mathcal{S M}\left(P_{0}\right)$ satisfies $w^{*} \succ_{u^{*}}^{Q} N\left(u^{*}\right)$. In particular, it follows that $\left\{\left\{u^{*}, w^{*}\right\}\right\}$ is not a stable set in $Q$. By statement (ii), from this and from the assumption that $\rho(q)$ does not exist, we infer that $u^{*} \succ_{w^{*}}^{Q} N\left(w^{*}\right)$ (either $u^{*} \succ_{w^{*}}^{P_{0}} u$ and we get $u^{*} \succ_{w^{*}}^{Q} N\left(w^{*}\right)$ directly from statement (ii), or $u \succeq_{w^{*}}^{P_{0}} u^{*}$ and by statement (ii) we get that either $u^{*} \succ_{w^{*}}^{Q} N\left(w^{*}\right)$ or $N\left(u^{*}\right)=w^{*}$-and we have already shown that in the latter case $N \notin \mathcal{S M}(Q))$. Thus, the pair $\left\{u^{*}, w^{*}\right\}$ is blocking $N$ in $Q$.

Summarizing, we have shown that no stable matching of $P_{0}$ is stable for $Q$.

Statement (iv). Assume that $\pi(q)$ does not exist and $\rho(q)$ exists but $\rho(q) \notin S$. Since $\pi(q)$ does not exist, by statement (i), for every stable matching $N \in \mathcal{S M}\left(P_{0}\right)$ it holds that $N\left(u^{*}\right)=w^{*}$ or $w^{*} \succ_{u^{*}}^{Q} N\left(u^{*}\right)$. This includes $M$, meaning that $M\left(u^{*}\right)=w^{*}$ or $w^{*} \succ_{u^{*}}^{Q} M\left(u^{*}\right)$.

We consider these two cases separately.

Case (1): $M\left(u^{*}\right)=w^{*}$. By statement (i), it follows that $w \succ_{u^{*}}^{P_{0}} w^{*}$. Further, since $q$ is a stable quadruple, there exists a stable matching $N \in \mathcal{S M}\left(P_{0}\right)$, such that $N\left(u^{*}\right)=w$ and $N(u)=w^{*}$. In $P_{0}$, since $u^{*}$ prefers $N$ to $M$, by Proposition 3.2, it must be the case that $w^{*}$ prefers $M$ to $N$, i.e., $u^{*} \succ_{w^{*}}^{P_{0}} u$. Thus, the rotation $\rho(q)$ (which, by our assumption, is guaranteed to exist) operates as follows: it changes the partner of $w^{*}$ from an agent that is less preferred than $u^{*}$ to $u^{*}$ or to an agent that is more preferred than $u^{*}$ (regarding $P_{0}$ ). Since $\rho(q) \notin S$, we infer that in matching $M$ agent $w^{*}$ obtains a partner that is less preferred than $u^{*}$, i.e., $u^{*} \succ_{w^{*}}^{P_{0}} M\left(w^{*}\right)$. This leads to a contradiction with $M\left(u^{*}\right)=w^{*}$.

Case (2): $w^{*} \succ_{u^{*}}^{Q} M\left(u^{*}\right)$. Towards a contradiction, suppose that $M$ is also stable for $Q$. This implies that $M\left(w^{*}\right) \succ_{w^{*}}^{Q} u^{*}$. By Observation 3.12(iii)(b), we have that $M\left(w^{*}\right) \succ_{w^{*}}^{P_{0}} u$. If $u \succ_{w^{*}}^{P_{0}} u^{*}$, then the rotation $\rho(q)$ changes the partner of $w^{*}$ from $u$ to some agent which is more preferred than $u$ (regarding the preferences in $P_{0}$ ). Since, according to $M$, agent $w^{*}$ already has a partner that is more preferred than $u$, we infer that $\rho(q)$ is the predecessor of some rotation in $S$, meaning that itself $\rho(q) \in S$ by the closedness of $S-$ a contradiction. If $u^{*} \succ_{w^{*}}^{P_{0}} u$, then $\succ_{w^{*}}^{P_{0}}=\succ_{w^{*}}^{Q}$ by Observation 3.12(iii), and so we get that $M\left(w^{*}\right) \succ_{w^{*}}^{P_{0}} u^{*} \succ_{w^{*}}^{P_{0}} u$ because $M\left(w^{*}\right) \succ_{w^{*}}^{Q} u^{*}$. In this case, rotation $\rho(q)$ changes the partner of $w^{*}$ from an agent that is less preferred than $u^{*}$ to $u^{*}$ or an agent who is more preferred than $u^{*}$. However, since in $M$ agent $w^{*}$ already has a partner who is preferred over $u^{*}$, we again infer that $\rho(q) \in S$ - a contradiction.

Summarizing, we conclude that $M \notin \mathcal{S M}(Q)$.

Statement (v). Let us assume that $\rho(q)$ exists and $\rho(q) \in S$.

By Lemma 3.13, except $\left\{u^{*}, w^{*}\right\}$, no other unmatched pair with respect to $M$ could be blocking 
$Q$. In the following, we claim that $\left\{u^{*}, w^{*}\right\}$ is not blocking $Q$, implying that $M$ is stable for $Q$. We distinguish between two cases.

If $u^{*} \succ_{w^{*}}^{P_{0}} u$, then by the definition of $\rho(q)$ and since $\rho(q) \in S$, it follows that $M\left(w^{*}\right)=u^{*}$ or $M\left(w^{*}\right) \succ_{w^{*}}^{P_{0}} u^{*}$. Moreover, by Observation 3.12(iii) we have that $\succ_{w^{*}}^{Q}=\succ_{w^{*}}^{Q}$, implying $M\left(w^{*}\right)=u^{*}$ or $M\left(w^{*}\right) \succ_{w^{*}}^{Q} u^{*}$. Thus, $\left\{u^{*}, w^{*}\right\}$ cannot be blocking $M$ in $Q$.

If $u \succ_{w^{*}}^{P_{0}} u^{*}$, then by the definition of $\rho(q)$ and since $\rho(q) \in S$, it follows that $M\left(w^{*}\right) \succ_{w^{*}}^{P_{0}} u$. Moreover, by Observation 3.12(iii)(b) we obtain that $M\left(w^{*}\right) \succ_{w^{*}}^{Q} u^{*}$. Thus, $\left\{u^{*}, w^{*}\right\}$ cannot be blocking $M$ in $Q$.

Statement (vi). Let us assume that $\pi(q)$ exists and $\pi(q) \notin S$. By Lemma 3.13, except $\left\{u^{*}, w^{*}\right\}$, no other unmatched pair with respect to $M$ could be blocking $Q$. In the following, we claim that $\left\{u^{*}, w^{*}\right\}$ is not blocking $Q$, which implies that $M$ is stable for $Q$. We distinguish between two cases.

If $w^{*} \succ_{u^{*}}^{P_{0}} w$, then by the definition of $\pi(q)$ and since $\pi(q) \notin S$, it follows that $M\left(u^{*}\right)=w^{*}$ or $M\left(u^{*}\right) \succ_{u^{*}}^{P_{0}} w^{*}$. Moreover, by Observation 3.12(ii) we have that $\succ_{u^{*}}^{Q}=\succ_{u^{*}}^{Q}$, implying $M\left(u^{*}\right)=w^{*}$ or $M\left(u^{*}\right) \succ_{u^{*}}^{Q} w^{*}$. Thus, $\left\{u^{*}, w^{*}\right\}$ cannot be blocking $M$ in $Q$.

If $w \succ_{u^{*}}^{P_{0}} w^{*}$, then by the definition of $\pi(q)$ and since $\pi(q) \notin S$, it follows that $M\left(u^{*}\right) \succ_{u^{*}}^{P_{0}} w$. Moreover, by Observation 3.12(ii)(b) we obtain that $M\left(u^{*}\right) \succ_{u^{*}}^{Q} w^{*}$. Thus, $\left\{u^{*}, w^{*}\right\}$ cannot be blocking $M$ in $Q$.

Statement (vii). Assume that $\pi(q)$ exists and $\pi(q) \in S$ and either $\rho(q)$ does not exist or it exists but $\rho(q) \notin S$.

Suppose, for the sake of contradiction, that $M$ is stable for $Q$. We distinguish between three cases, in each case obtaining a contradiction.

Case 1: $\boldsymbol{w}^{*} \succ_{\boldsymbol{u}^{*}}^{\boldsymbol{P}_{\mathbf{0}}} \boldsymbol{w}$. By the definition of $\pi(q)$ and since $\pi(q) \in S$, referencing Proposition 3.9(i), it follows that $w^{*} \succ_{u^{*}}^{P_{0}} M\left(u^{*}\right)$. Thus, $w^{*} \succ_{u^{*}}^{Q} M\left(u^{*}\right)$ because $\succ_{u^{*}}^{P_{0}}=\succ_{u^{*}}^{Q}$ (by Observation 3.12(ii)). In particular, this implies that $\left\{u^{*}, w^{*}\right\}$ is an unmatched pair in $M$. By assumption that $M$ is stable for $Q$, we must have that

$$
M\left(w^{*}\right) \succ_{w^{*}}^{Q} u^{*}
$$

If $u^{*} \succ_{w^{*}}^{P_{0}} u$, then by Observation 3.12(iii), we have $M\left(w^{*}\right) \succ_{w^{*}}^{P_{0}} u^{*} \succ_{w^{*}}^{P_{0}} u$. By the fact that $\left\{u, w^{*}\right\}$ is in some stable matching (recall that $q$ is a stable quadruple), there are two stable matchings, where $w^{*}$ obtains a partner (namely, $u$ ) who is less preferred than $u^{*}$, and a partner (namely, $M\left(w^{*}\right)$ ) who is more preferred than $u^{*}$. This implies that $\rho(q)$ exists and that $\rho(q) \in S$-a contradiction.

If $u \succ_{w^{*}}^{P_{0}} u^{*}$, then by Observation 3.12(iii) and by (7), we have that $M\left(w^{*}\right) \succ_{w^{*}}^{P_{0}} u \succ_{w^{*}}^{P_{0}} \succ u^{*}$. Again, by the fact that $\left\{u, w^{*}\right\}$ is in some stable matching (recall that $q$ is a stable quadruple), there are two stable matchings, where $w^{*}$ obtains partner $u$, and a partner (namely, $M\left(w^{*}\right)$ ) who is more preferred than $u$. This implies that $\rho(q)$ exists such that $\rho(q) \in S$-a contradiction.

Case 2: $\boldsymbol{w} \succ_{\boldsymbol{u}^{*}}^{\boldsymbol{P}_{\mathbf{*}}} \boldsymbol{w}^{*}$ and $\boldsymbol{M}\left(\boldsymbol{u}^{*}\right) \neq \boldsymbol{w}^{*}$. If we can show that $w^{*} \succ_{u^{*}}^{Q} M\left(u^{*}\right)$, then we can use the same reasoning as we did for the first case to show the same contradiction. Thus it suffices to prove that $w^{*} \succ_{u^{*}}^{Q} M\left(u^{*}\right)$.

By the definition of $\pi(q)$ and since $\pi(q) \in S$, referencing Proposition 3.9(i), it follows that $M\left(u^{*}\right)=w$ or $w \succ_{u^{*}}^{P_{0}} M\left(u^{*}\right)$, and thus $w^{*} \succ_{u^{*}}^{Q} M\left(u^{*}\right)$ because $M\left(u^{*}\right) \neq w^{*}$ (by assumption) and $w^{*} \succ_{u^{*}}^{Q} w$. This finishes the proof for the second case.

Case 3: $\boldsymbol{w} \succ_{\boldsymbol{u}^{*}}^{\boldsymbol{P}_{0}} \boldsymbol{w}^{*}$ and $\boldsymbol{M}\left(\boldsymbol{u}^{*}\right)=\boldsymbol{w}^{*}$. Since $q$ is a stable quadruple, $\left\{u^{*}, w\right\}$ and $\left\{u, w^{*}\right\}$ exist in some stable matching of $P_{0}$, say $N$. Thus, $P_{0}$ admits two different stable matchings $M$ and $N$, 
where $M\left(u^{*}\right)=w^{*}, N\left(u^{*}\right)=w$, and $N\left(w^{*}\right)=u$. By the precondition that $w \succ_{u^{*}}^{P_{0}} w^{*}$ and by Proposition 3.2, we must have that $u^{*} \succ_{w^{*}} u$ (i.e. $w^{*}$ prefers $M$ to $N$ ). In particular, this means that there must be a rotation which changes the partner of $w^{*}$ from one that is less preferred than $u^{*}$ to agent $u^{*}$. Thus, $\rho(q)$ exists and must be in $S$-a contradiction.

\subsection{Polynomial-Time Algorithms for Robust Matchings}

We now first present an $O\left(n^{4}\right)$-time algorithm for finding a robust matching if it exists. Then we use a Linear Programming (LP) formulation to show that perfect robust matchings and robust matchings with minimum egalitarian cost can be found in polynomial time if they exist. Both approaches crucially rely on (a) the one-to-one correspondence between the stable matchings and the closed subsets of the rotation poset [18, Chapter 3.7], (b) the implications between the presence of the two rotations $\pi(q)$ and $\rho(q)$ of stable quadruples $q$ derived in Lemma 3.19, and (c) the fact that all stable quadruples can be computed in $O\left(n^{4}\right)$ time. The proof for (c) is roughly by iterating over all possible rotations and building a lookup table that stores for all pairs $(x, y) \in U \times W$ of agents a constant number of rotations that make the partner of $x$ less preferred to $y$ or more preferred to $y$. For given stable quadruple $q$, rotations $\pi(q)$ and $\rho(q)$ can then be looked up in the table. We state this observation for reference below.

Proposition 3.20. Determining all stable quadruples $q$ and their respective rotations $\pi(q)$ and $\rho(q)$ as defined in Definition 3.16 can be done in $O\left(n^{4}\right)$ time.

Proof. By Proposition 3.1 and by Proposition 3.9(ii), all $O\left(n^{4}\right)$ stable quadruples can be found in $O\left(n^{4}\right)$ time.

For each stable quadruple $q$, we show how to find $\pi(q)$ and $\rho(q)$, in $O(1)$ time for a given stable quadruple $q$. We build in $O\left(n^{4}\right)$ time a size- $O\left(n^{2}\right)$ look-up table $T$ to store for each ordered pair $(x, y) \in U \times W$ the following up to six rotations:

1. Let $\sigma_{1}(x, y)$ denote the rotation which changes the partner of $x$ from someone who is more preferred than $y$ to $y$.

Formally, $\sigma_{1}(x, y):=\left(\left(u_{0}^{\prime}, w_{0}^{\prime}\right), \ldots,\left(u_{r-1}^{\prime}, w_{r-1}^{\prime}\right)\right)$ such that $x=u_{i}^{\prime}$ and $y=w_{i+1}^{\prime}$ for some $i \in$ $\{0, \ldots, r-1\}$. Note that the uniqueness of this rotation is guaranteed by Proposition 3.6(iii).

2. Let $\sigma_{2}(x, y)$ denote the rotation which changes the partner of $x$ from someone who is more preferred than $y$ to someone who is less preferred than $y$.

Formally, $\sigma_{2}(x, y):=\left(\left(u_{0}^{\prime}, w_{0}^{\prime}\right), \ldots,\left(u_{r-1}^{\prime}, w_{r-1}^{\prime}\right)\right)$ such that $x=u_{i}^{\prime}$ and $w_{i}^{\prime} \succ_{x} y \succ_{x} w_{i+1}^{\prime}$ for some $i \in\{0, \ldots, r-1\}$. Note that the uniqueness of this rotation is guaranteed by Proposition 3.6(ii).

3. Let $\sigma_{3}(x, y)$ denote the rotation which changes the partner of $x$ from $y$ to someone who is less preferred than $y$.

Formally, $\sigma_{3}(x, y):=\left(\left(u_{0}^{\prime}, w_{0}^{\prime}\right), \ldots,\left(u_{r-1}^{\prime}, w_{r-1}^{\prime}\right)\right)$ such that $x=u_{i}^{\prime}$ and $y=w_{i}^{\prime}$ for some $i \in$ $\{0, \ldots, r-1\}$. Note that the uniqueness of this rotation is guaranteed by Proposition 3.6(ii).

Moreover, the existence of $\sigma_{2}(x, y)$ precludes the existence of $\sigma_{1}(x, y)$ and $\sigma_{3}(x, y)$ because $\sigma_{2}(x, y)$ implies that $\{x, y\}$ is not in any stable matching while $\sigma_{1}(x, y)$ or $\sigma_{3}(x, y)$ implies that $\{x, y\}$ is some stable matching.

4. Let $\tau_{1}(y, x)$ denote the rotation which changes the partner of $y$ from someone who is less preferred than $x$ to $x$.

Formally, $\tau_{1}(y, x):=\left(\left(u_{0}^{\prime}, w_{0}^{\prime}\right), \ldots,\left(u_{r-1}^{\prime}, w_{r-1}^{\prime}\right)\right)$ such that $y=w_{i}^{\prime}$ and $x=u_{i-1}^{\prime}$ for some $i \in$ $\{0, \ldots, r-1\}$. Note that the uniqueness of this rotation is guaranteed by Proposition 3.6(iii). 

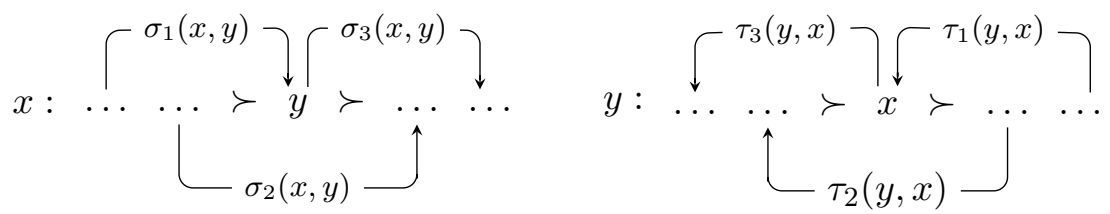

Figure 1: Illustration for the six rotations defined in the proof of Proposition 3.20.

5. Let $\tau_{2}(y, x)$ denote the rotation which changes the partner of $y$ from someone who is less preferred than $x$ to someone who is more preferred than $x$.

Formally, $\tau_{2}(y, x):=\left(\left(u_{0}^{\prime}, w_{0}^{\prime}\right), \ldots,\left(u_{r-1}^{\prime}, w_{r-1}^{\prime}\right)\right)$ such that $y=w_{i}^{\prime}$ and $u_{i-1}^{\prime} \succ_{y} x \succ_{y} u_{i}^{\prime}$ for some $i \in\{0, \ldots, r-1\}$. Note that the uniqueness of this rotation is guaranteed by Proposition 3.6(iv).

6 . Let $\tau_{3}(x, y)$ denote the rotation which changes the partner of $y$ from $x$ to someone who is more preferred than $x$.

Formally, $\tau_{1}(y, x):=\left(\left(u_{0}^{\prime}, w_{0}^{\prime}\right), \ldots,\left(u_{r-1}^{\prime}, w_{r-1}^{\prime}\right)\right)$ such that $y=w_{i}^{\prime}$ and $x=u_{i}^{\prime}$ for some $i \in$ $\{0, \ldots, r-1\}$. Note that the uniqueness of this rotation is guaranteed by Proposition 3.6(iv). Moreover, the existence of $\tau_{2}(y, x)$ precludes the existence of $\tau_{1}(y, x)$ and $\tau_{3}(y, x)$ because $\tau_{2}(y, x)$ implies that $\{x, y\}$ is not in any stable matching while $\tau_{1}(y, x)$ or $\tau_{3}(y, x)$ implies that $\{x, y\}$ is some stable matching.

Figure 1 illustrates the six rotations we have just defined.

Now, we continue with the determination of $\rho(q)$ and $\pi(q)$. Let $q=\left(u^{*}, w^{*}, u, w\right)$.

$$
\begin{aligned}
& \pi(q):= \begin{cases}\sigma_{2}\left(u^{*}, w^{*}\right), & \text { if } w^{*} \succ_{u^{*}} w \text { and } \sigma_{2}\left(u^{*}, w^{*}\right) \text { exists, } \\
\sigma_{3}\left(u^{*}, w^{*}\right), & \text { if } w^{*} \succ_{u^{*}} w \text { and } \sigma_{3}\left(u^{*}, w^{*}\right) \text { exists, } \\
\sigma_{2}\left(u^{*}, w\right), & \text { if } w \succ_{u^{*}} w^{*} \text { and } \sigma_{1}\left(u^{*}, w\right) \text { exists, } \\
\text { undefined, } & \text { otherwise. }\end{cases} \\
& \rho(q):= \begin{cases}\tau_{1}\left(w^{*}, u^{*}\right), & \text { if } u^{*} \succ_{w^{*}} u \text { and } \tau_{1}\left(w^{*}, u^{*}\right) \text { exists, } \\
\tau_{2}\left(w^{*}, u^{*}\right), & \text { if } u^{*} \succ_{w^{*}} u \text { and } \tau_{2}\left(w^{*}, u^{*}\right) \text { exists, } \\
\tau_{3}\left(w^{*}, u\right), & \text { if } u \succ_{u^{*}} u^{*} \text { and } \tau_{3}\left(w^{*}, u\right) \text { exists, } \\
\text { undefined, } & \text { otherwise. }\end{cases}
\end{aligned}
$$

One can verify that the above construction corresponds to Definition 3.16. Since there are $O\left(n^{2}\right)$ rotations and $n^{2}$ ordered pairs, the whole table, containing $O\left(n^{2}\right)$ entries, can be determined in $O\left(n^{4}\right)$ time. (Note that, for a given rotation $\rho$, we can first find the agents who are affected by the rotation in $O(n)$ time, and, for each of the affected agents $z$, find in $O(n)$ time all the pairs $(z, w)$ such that $\rho$ needs to be added to the table entry of $(z, w)$.) After computing the table, we can determine in constant time the two rotations $\pi(q)$ and $\rho(q)$ for each $q$ from the $O\left(n^{4}\right)$ stable quadruples, by looking up into the table. In total, the running time is $O\left(n^{4}\right)$.

(of Proposition 3.20) $\diamond$

We now prove our main result for the RoBUst MATCHING problem.

Theorem 3.21. Given an instance of RoBust MATCHING with $2 n$ agents, in $O\left(n^{4}\right)$ time we can either find a d-robust matching or correctly report that no such matching exists.

Proof. Our approach is described in Algorithm 1. Let $P$ be the profile in the given instance. 


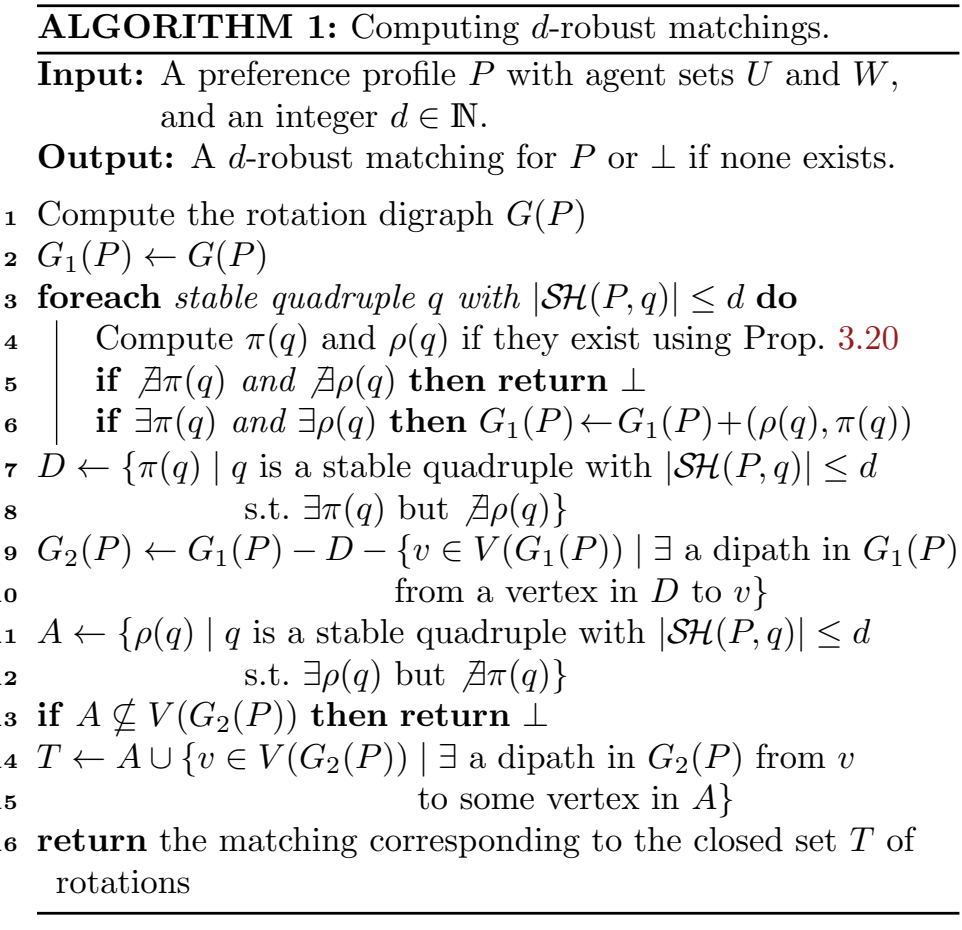

To obtain an $O\left(n^{4}\right)$ algorithm we work with the rotation digraph $G(P)$ (see Definition 3.7 and Proposition 3.9). Call a vertex subset $S$ in a directed graph $G$ closed, if there is no arc in $G$ pointing outwards from $S$. Recall that a stable matching for $P$ corresponds to a closed subset of the rotations in the rotation poset, i.e., a closed vertex subset $S$ of $G(P)$. Intuitively, Algorithm 1 first adds arcs to $G(P)$ in lines 2 to 6 that model implications between rotations contained in $d$-robust matchings given in Lemma 3.19. Then, it removes rotations from $G(P)$ that cannot occur in $d$-robust matchings according to Lemma 3.19 in lines 7 and 9. Finally, it checks in line 14 whether there is a closed subset of rotations which contains the required rotations for $d$-robust matchings according to Lemma 3.19.

We now prove the correctness and then analyze the running time. Below, when referring to $G_{1}(P)$, we mean the graph $G_{1}(P)$ obtained from $G(P)$ after line 6 and by $G_{2}(P)$ we mean the graph obtained after line 10 .

We claim that, if Algorithm 1 returns something different from $\perp$, then it is a $d$-robust matching. We first show that the set $T$ computed in line 14 is a closed subset of rotations in the rotation poset: Clearly, $T$ is closed in $G_{2}(P)$. Since $G_{2}(P)$ is obtained from $G_{1}(P)$ by removing vertices together with all of their successors, $T$ is closed in $G_{1}(P)$ as well. Since $G_{1}(P)$ is obtained from $G(P)$ by adding arcs, $T$ is closed in $G(P)$, implying the claim. By Proposition 3.9, there is a stable matching $M$ for $P$ associated with $T$.

Since $T$ is a closed subset, Lemma 3.19 (iv) to (vii) apply. We now verify that, for each stable quadruple $q$ with $|\mathcal{S H}(P, q)| \leq d$, we have $M \in \mathcal{S M}(P[\mathcal{S H}(P, q)]$ ) (recall that $P[\mathcal{S H}(P, q)]$ is the profile obtained from $P$ by performing the swaps in $\mathcal{S H}(P, q)$ ). By Lemma 3.15 it then follows that $M$ is $d$-robust.

Let $q$ be a stable quadruple of $P$ such that $|\mathcal{S H}(P, q)| \leq d$. Suppose that $\rho(q)$ exists. If $\pi(q)$ does not exist, then $\rho(q) \in T$ by line 11 and line 14, and thus $M \in \mathcal{S M}(P[\mathcal{S H}(P, q)])$ by Lemma $3.19(\mathrm{v})$. If $\pi(q)$ exists, then, since $T$ is closed and by line 6 , either $\pi(q) \notin T$, giving $M \in \mathcal{S M}(P[\mathcal{S H}(P, q)])$ by Lemma 3.19 (vi), or $\rho(q) \in T$, giving $M \in \mathcal{S M}(P[\mathcal{S H}(P, q)])$ by Lemma 3.19 (v). Now suppose that $\rho(q)$ does not exist. Then, $\pi(q)$ exists because otherwise we would have returned $\perp$ in line 5 . By lines 7 and $9, \pi(q) \notin T$, giving $M \in \mathcal{S M}(P[\mathcal{S H}(P, q)])$ by Lemma 3.19 (vi). Thus, indeed the returned matching is $d$-robust.

It remains to show that a $d$-robust matching is returned if there is a $d$-robust matching $M$ for $P$. By the above, it suffices to show that $\perp$ is not returned in lines 5 and 13. By Lemma 3.15, $M \in \mathcal{S M}(P[\mathcal{S H}(P, q)])$ for each stable quadruple $q$ with $|\mathcal{S H}(P, q)| \leq d$. Thus, by Lemma 3.19 (iii) at least one of $\rho(q)$ and $\pi(q)$ exists, meaning that $\perp$ cannot be returned in line 5 . If $\perp$ was returned 
due to line 13, then there is a stable quadruple $q$ with $|\mathcal{S H}(P, q)| \leq d$ such that $\pi(q)$ does not exist and $\rho(q)$ exists and, furthermore, $\rho(q) \in V\left(G_{1}(P)\right) \backslash V\left(G_{2}(P)\right)$. Let $S$ be the closed subset of rotations in $G(P)$ associated with $M$. By Lemma 3.19 (iv), $\rho(q) \in S$. Since $\rho(q) \notin V\left(G_{2}(P)\right)$, by lines 7 and 9 , there is a stable quadruple $q^{\prime}$ with $\left|\mathcal{S H}\left(P, q^{\prime}\right)\right| \leq d$ such that $\pi\left(q^{\prime}\right) \in D$ and there is a path (possibly of length zero) from $\pi\left(q^{\prime}\right)$ to $\rho(q)$ in $G_{1}(P)$. Since $\rho(q) \in S$, thus also $\pi\left(q^{\prime}\right) \in S$. By line $9, \rho\left(q^{\prime}\right)$ does not exist. Thus, by Lemma 3.19 (vii) $M \notin \mathcal{S M}\left(P\left[\mathcal{S H}\left(P, q^{\prime}\right)\right]\right)$, a contradiction to $M$ being $d$-robust. Thus, indeed a $d$-robust matching is returned if there is one.

The running time of $O\left(n^{4}\right)$ can be obtained as follows. By Proposition 3.9, the rotation digraph in line 1 can be computed in $O\left(n^{2}\right)$ time. Lines 3 and 4 can be carried out in $O\left(n^{4}\right)$ time by Proposition 3.20. Thus, clearly, lines 2 to 6 can be carried out in $O\left(n^{4}\right)$ time. Lines $7-9$ can be done in $O\left(n^{4}\right)$ because $G(P)$ contains $O\left(n^{2}\right)$ vertices. Analogously, lines 11-16 take $O\left(n^{4}\right)$ time.

Example 3.22. To illustrate Algorithm 1, consider the profile $P$ given in Example 1.1 and let $d=1$. $P$ admits three rotations, $\pi_{1}=\left(\left(u_{1}, w_{2}\right),\left(u_{2}, w_{3}\right),\left(u_{3}, w_{1}\right),\left(u_{4}, w_{1}\right)\right), \pi_{2}=\left(\left(u_{1}, w_{3}\right),\left(u_{3}, w_{1}\right)\right)$, and $\pi_{3}=\left(\left(u_{2}, w_{4}\right),\left(u_{4}, w_{2}\right)\right)$. There are ten stable quadruples for $d=1$. The corresponding $\pi(q)$ and $\rho(q)$ are summarized in the lower left table.

\begin{tabular}{c|cc}
\hline $\begin{array}{c}\text { Stable quadruple } q \\
\text { with }|\mathcal{S H}(P, q)| \leq 1\end{array}$ & $\pi(q)$ & $\rho(q)$ \\
\hline$\left(u_{1}, w_{1}, u_{3}, w_{3}\right)$ & $\pi_{1}$ & $\pi_{2}$ \\
$\left(u_{1}, w_{2}, u_{4}, w_{1}\right)$ & $\pi_{1}$ & $\pi_{3}$ \\
$\left(u_{1}, w_{2}, u_{4}, w_{3}\right)$ & $\pi_{1}$ & $\pi_{3}$ \\
$\left(u_{1}, w_{3}, u_{2}, w_{2}\right)$ & no & $\pi_{1}$ \\
$\left(u_{1}, w_{4}, u_{2}, w_{1}\right)$ & $\pi_{2}$ & $\pi_{3}$ \\
$\left(u_{2}, w_{1}, u_{3}, w_{2}\right)$ & $\pi_{3}$ & $\pi_{2}$ \\
$\left(u_{2}, w_{2}, u_{4}, w_{4}\right)$ & $\pi_{1}$ & $\pi_{3}$ \\
$\left(u_{2}, w_{4}, u_{3}, w_{3}\right)$ & no & $\pi_{1}$ \\
$\left(u_{3}, w_{1}, u_{4}, w_{4}\right)$ & no & $\pi_{1}$ \\
$\left(u_{3}, w_{2}, u_{4}, w_{3}\right)$ & $\pi_{2}$ & $\pi_{3}$ \\
\hline
\end{tabular}

The digraphs $G(P)$ and $G_{1}(P)=G_{2}(P)$ constructed in Algorithm 1 are depicted in the lower right figure.

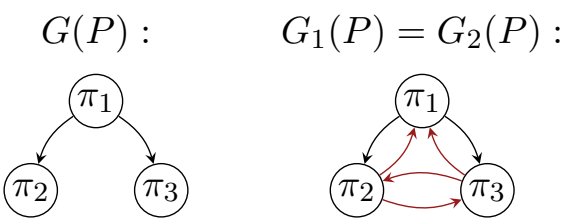

One can verify that $A=\left\{\pi_{1}\right\}$ (see rows $4,8,9$ in the table). $T=\left\{\pi_{1}, \pi_{2}, \pi_{3}\right\}$ is the only closed set in $G_{2}$ that includes $\pi_{1}$, which corresponds to $M_{2}$. Indeed our algorithm will return $M_{2}$ (see Example 1.1) as the only 1-robust matching.

Now we turn to the problem variants where we look for a perfect $d$-robust matching or one with minimum egalitarian cost. Our polynomial-time algorithm for these variants builds on a Linear Programming (LP) formulation which finds a stable matching. This LP formulation in turn is based on the one-to-one correspondence between the stable matchings and the closed subsets of the rotation poset [18, Chapter 3.7]. A crucial property of this formulation is that its constraint matrix is totally unimodular. Hence, each extreme point of the polytope defined by this formulation is integral.

The LP formulation is as follows. Let $P_{0}$ be a preference profile with two disjoint sets, $U$ and $W$, each containing $n$ agents. Let $R(P)$ be the set of rotations for $P_{0}$ and let $G\left(P_{0}\right)$ with arc set $E\left(P_{0}\right)$ be the rotation digraph of $P_{0}$ regarding the precedence relation $\triangleright^{P_{0}}$; by Proposition 3.9(ii), both the rotation set $R\left(P_{0}\right)$ and the rotation digraph $G\left(P_{0}\right)$ can be computed in $O\left(n^{2}\right)$ time. For each rotation $\rho \in R\left(P_{0}\right)$, we introduce a variable $x_{\rho}$ with box constraints $0 \leq x_{\rho} \leq 1$, where $x_{\rho}=1$ will correspond to adding $\rho$ to the solution subset while $x_{\rho}=0$ means that $\rho$ will not be taken into the subset. By Gusfield and Irving [18, Chapter 3.7], the constraint matrix of the constraints

$$
\begin{aligned}
x_{\rho}-x_{\pi} \leq 0, & \forall \pi, \rho \in R\left(P_{0}\right) \text { with }(\pi, \rho) \in E\left(P_{0}\right), \\
0 \leq x_{\rho} \leq 1, & \forall \rho \in R\left(P_{0}\right),
\end{aligned}
$$


is totally unimodular and thus there is a solution in which each variable takes either value zero or one. In this way, the set $S=\left\{\rho \mid x_{\rho}=1\right\}$, defined by including exactly those rotations whose variable values are set to one is closed under the rotation poset and thus defines a stable matching.

Before we state our main result for the RoBust MATCHing problem, we recall a condition which ensures that an LP formulation gives an integral solution.

Proposition 3.23 ([7]). If $A \in\{-1,0,+1\}^{\hat{n} \times \hat{m}}$ and $b \in \mathbb{Z}^{\hat{m}}$ such that each row in $A$ has at most one +1 and at most one -1 , then $A$ is totally unimodular, and every extreme point of the system $A x \leq b, x \in \mathbb{N}_{0}^{\hat{m}}$ is integral.

Theorem 3.24. Finding a d-robust perfect matching and finding a d-robust matching with minimum egalitarian cost, if they exist, can both be done in polynomial time.

Proof. Following Lemma 3.19, we will add some additional constraints to the LP given by (LP1) and (LP2), which results in an LP whose constraint matrix remains totally unimodular (see Proposition 3.23). To determine whether there is a $d$-robust matching for our instance, we need to consider every possible profile that differs from the original profile by at most $d$ swaps. For $2 n$ agents, there are, however, $(2 n)^{O(d)}$ such profiles. To avoid this, we characterize these profiles by stable quadruples, using Lemma 3.15. To achieve this, we compute for each stable quadruple $q$ with $\left|\mathcal{S H}\left(P_{0}, q\right)\right| \leq d$ the two specific rotations $\pi(q)$ and $\rho(q)$ as defined in Definition 3.16.

As already discussed, $\pi(q)$ and $\rho(q)$ may not exist. If they exist, then by Lemma 3.18 they are unique. Moreover, by Lemma 3.19(iii), we may assume that at least one of $\pi(q)$ and $\rho(q)$ exist as otherwise $\mathcal{S M}(P) \cap \mathcal{S M}(P[\mathcal{S H}(P, q)])=\emptyset$, implying that $P$ does not admit a $d$-robust matching. We distinguish between three cases, in each case describing how to add some constraints to the LP defined above.

Case (1): Both $\boldsymbol{\pi}(\boldsymbol{q})$ and $\boldsymbol{\rho}(\boldsymbol{q})$ exist. Add the constraint $x_{\pi(q)}-x_{\rho(q)} \leq 0$.

By Lemma 3.19, statements (v), (vi), and (vii), the stable matching defined according to a closed subset is stable in $P\left[\mathcal{S H}\left(P_{0}, q\right)\right]$ if and only if $x_{\rho(q)}=1$ or $x_{\pi(q)}=0$.

Case (2): $\boldsymbol{\pi}(\boldsymbol{q})$ exists but $\boldsymbol{\rho}(\boldsymbol{q})$ does not. Add the constraint $x_{\pi(q)}=0$

The above constraint is justified by Lemma 3.19(vii).

Case (3): $\boldsymbol{\pi}(\boldsymbol{q})$ does not exist but $\boldsymbol{\rho}(\boldsymbol{q})$ exists. Add the constraint $x_{\rho(q)}=1$

This constraint is justified by Lemma 3.19, statements (iv) and (v).

Note that in each of the three cases, we add to the constraint matrix a row which has at most one +1 , at most one -1 and the remaining values are all 0 s. Thus, we can infer by Proposition 3.23 that the resulting constraint matrix is still totally unimodular and all primal solutions to our problem are integral. Since the matrix has $O\left(n^{4}\right)$ rows and $O\left(n^{2}\right)$ columns, solving the thus constructed LP can be done in polynomial time.

Since all stable matchings match the same set of agents (Proposition 2.3), it is apparent from the above LP that finding a $d$-robust and perfect matching if it exists can be done in polynomial time. Finding a $d$-robust matching, if one exists, with minimum egalitarian cost can also be done in polynomial time by the following: For each rotation $\rho$ we can compute how adding $\rho$ to a stable matching changes its egalitarian score. Then, it is sufficient to add an appropriate optimization objective to the LP constructed above. 


\section{Robustness and Preferences with Ties: NP-hardness}

When the input preferences may contain ties, we consider a swap to be a pair of two agents that belong to two neighboring tied classes. For the case with ties, the preference list $\succeq$ of each agent may be expressed as a transitive and complete binary relation on the set of the agents who she finds acceptable. The expression " $x \succeq_{i} y$ " means that $i$ weakly prefers $x$ over $y$ (i.e. $x$ is better or as good as $y$ ). We use $\succ_{i}$ to denote the asymmetric part (i.e. $x \succeq_{i} y$ and $\left.\neg\left(y \succeq_{i} x\right)\right)$ and $\sim_{i}$ to denote the symmetric part of $\succeq_{i}$ (i.e. $x \succeq_{i} y$ and $\left.y \succeq_{i} x\right)$.

Formally, we define the swap distance $\tau\left(\succeq_{i}, \succeq_{i^{\prime}}\right)$ between two preference lists with ties as follows.

$$
\delta\left(\succeq_{i}, \succeq_{i^{\prime}}\right):= \begin{cases}\infty, & \text { if } \succeq_{i} \text { and } \succeq_{i^{\prime}} \text { have } \\ \left|\left\{(x, y) \in \succ_{i} \mid(y, x) \in \succeq_{i^{\prime}}\right\}\right|+\left|\left\{(x, y) \in \sim_{i} \mid(x, y) \notin \sim_{i}\right\}\right|, & \text { different acceptable sets, } \\ \text { otherwise. }\end{cases}
$$

In particular, if an agent has a preference list $(a, b, c)$, meaning that all three agents are tied on the first position, then moving to the list $c \succ(a, b)$ requires two swaps (swapping $a$ with $c$ and $b$ with c). Finding a stable matching can be done in $O\left(n^{2}\right)$ time even when ties are present [19]. In contrast, presence of ties makes RoBUst MATching NP-hard:

Theorem 4.1. Robust Matching with ties is NP-hard even when the number d of swaps allowed is one.

Proof. We reduce from Independent Set. Let $I=(G, k)$ be an instance of Independent Set. Further, let $V(G)=\left\{v_{1}, \ldots, v_{n}\right\}$ and $E(G)=\left\{e_{1}, \ldots, e_{m}\right\}$ denote the set of vertices and the set of edges in $G$ respectively. Without loss of generality, we assume that the number $n$ of vertices in $V$ is at least three and the solution size $k$ is at least two. We construct an instance of RoBUst MATCHING with two sets of agents, $U$ and $W$, and with the number of allowed swaps equal to $d=1$.

Agent set $\boldsymbol{U}$. This set consists of the following $n+2 m+2$ agents.

$$
\begin{aligned}
& U=V \cup E \cup F \cup A, \text { where } \\
& V=\left\{v_{1}, \ldots, v_{n}\right\}, \\
& E=\left\{e_{1}, \ldots, e_{m}\right\}, \\
& F=\left\{f_{1}, \ldots, f_{m}\right\}, \\
& A=\left\{a_{0}, a_{1}\right\} .
\end{aligned}
$$

Agent set $\boldsymbol{W}$. This set consists of the following $n+2 m+2$ agents.

$$
\begin{aligned}
W & =T \cup S \cup E_{V} \cup B, \text { where } \\
T & =\left\{t_{1}, \ldots, t_{n-k}\right\}, \\
S & =\left\{s_{1}, \ldots, s_{k}\right\}, \\
E_{V} & =\left\{e_{\ell}^{v_{i}}, e_{\ell}^{v_{j}} \mid e_{\ell} \in E(G) \text { with } e_{\ell}=\left\{v_{i}, v_{j}\right\}\right\}, \\
B & =\left\{b_{0}, b_{1}\right\} .
\end{aligned}
$$

Note that we use $v_{i}\left(\right.$ resp. $e_{\ell}$ ) for both a vertex and its corresponding vertex agent (resp. an edge and its corresponding edge agent). It will, however, be clear from the context what we are referring to. 
The preference lists of these agents are defined as follows, where $[\star]$ means that the elements in $\star$ are ranked in an arbitrary but fixed order, while $(\star)$ means that the elements in $\star$ are tied. The symbol ... at the end of each preference list denotes an arbitrary but fixed order of the remaining not mentioned agents.

Preference lists of the agents from $U$.

$$
\begin{aligned}
& \forall i \in[n] \quad v_{i}:(T) \succ b_{0} \succ\left[\left\{e_{\ell}^{v_{i}} \mid e_{\ell} \in E(G) \text { such that } v_{i} \in e_{\ell}\right\}\right] \succ(S) \succ \ldots, \\
& \forall \ell \in[m] \text { with } e_{\ell}=\left\{v_{i}, v_{j}\right\} \quad e_{\ell}:\left(e_{\ell}^{v_{i}}, e_{\ell}^{v_{j}}, b_{0}\right) \succ \ldots, \\
& \forall \ell \in[m] \text { with } e_{\ell}=\left\{v_{i}, v_{j}\right\} \text { and } i<j \quad f_{\ell}: e_{\ell}^{v_{j}} \succ e_{\ell}^{v_{i}} \succ b_{0} \succ \ldots, \\
& a_{0}: b_{0} \succ b_{1} \succ \ldots, \\
& a_{1}: b_{1} \succ b_{0} \succ \ldots
\end{aligned}
$$

\section{Preference lists of the agents in $W$.}

$$
\begin{aligned}
\forall i \in[n-k] & t_{i}:(V) \succ a_{0} \succ a_{1} \succ \ldots, \\
\forall i \in[k] & s_{i}:(V) \succ a_{0} \succ a_{1} \succ \ldots, \\
\forall \ell \in[m] \text { with } e_{\ell}=\left\{v_{i}, v_{j}\right\} & e_{\ell}^{v_{i}}: e_{\ell} \succ a_{0} \succ f_{\ell} \succ v_{i} \succ \ldots, \\
& e_{\ell}^{v_{j}}: e_{\ell} \succ a_{0} \succ f_{\ell} \succ v_{j} \succ \ldots, \\
& b_{0}: a_{0} \succ a_{1} \succ \ldots, \\
& b_{1}: a_{1} \succ a_{0} \succ \ldots
\end{aligned}
$$

We use $P$ to denote the above preference profile. Before we prove the correctness of our construction, we observe some properties which every stable matching must satisfy.

Claim 1. Every stable matching $M$ for $U$ and $W$ with respect to the initial preferences must satisfy the following.

1. Each agent $t_{i} \in T$ must be matched with an agent from $V$.

2. Each agent $a_{j} \in A$ must be matched with $b_{j}$.

3. For each edge $e_{\ell} \in E(G)$ with $e_{\ell}=\left\{v_{i}, v_{j}\right\}$, the agents $e_{\ell}$ and $f_{\ell}$ must have $\left\{M\left(e_{\ell}\right), M\left(f_{\ell}\right)\right\}=$ $\left\{e_{\ell}^{v_{i}}, e_{\ell}^{v_{j}}\right\}$.

4. Each agent $s_{i} \in S$ must be matched with an agent from $V$.

Proof. The first statement is straight-forward because every vertex agent $v_{i} \in V$ and every selector agent $t_{j} \in T$ rank each other at the first position.

Analogously, we obtain the second statement.

Now, consider an arbitrary edge $e_{\ell} \in E(G)$ and let $v_{i}$ and $v_{j}$ denote the endpoints of edge $e_{\ell}$. Since agent $a_{0}$ is already matched with $b_{0}$ we can neglect them from the preference lists of $e_{\ell}, f_{\ell}, e_{\ell}^{v_{i}}$, and $e_{\ell}^{v_{j}}$. Consequently, one can verify that the partners of $e_{\ell}$ and $f_{\ell}$ must be from $\left\{e_{\ell}^{v_{i}}, e_{\ell}^{v_{j}}\right\}$.

By the first three statement, there are $k$ agents left from $V$ who each must be matched with some agent from $S$ because every agent from $S$ ranks every agent from $V$ at the first position.

$($ of Claim 1) $\diamond$

We show that $G$ admits an independent set of size $k$ if and only if the profile $P$ has a stable matching $M$ that remains stable in each profile $P^{\prime}$ that differs from $P$ by at most one swap. 
For the "only if" direction, let $V^{\prime}=\left\{v_{i_{1}}, \ldots, v_{i_{k}}\right\}$ be an independent set of $k$ vertices with $i_{1}<$ $i_{2}<\ldots<i_{k}$. For the sake of convenience, let $V \backslash V^{\prime}=\left\{v_{j_{1}}, \ldots, v_{j_{n-k}}\right\}$ with $j_{1}<j_{2}<\ldots<j_{n-k}$.

We claim that the following perfect matching $M$ is stable in every profile which differs from the original one by at most one swap.

$$
\begin{aligned}
M= & \left\{\left\{a_{0}, b_{0}\right\},\left\{a_{1}, b_{1}\right\}\right\} \cup\left\{\left\{\left\{s_{r}, v_{i_{r}}\right\} \mid v_{i_{r}} \in V^{\prime}\right\}\right\} \cup \\
& \left\{\left\{t_{r}, v_{j_{r}}\right\} \mid v_{j_{r}} \in V \backslash V^{\prime}\right\} \cup \\
& \left\{\left\{e_{\ell}, e_{\ell}^{v_{i}}\right\},\left\{f_{\ell}, e^{v_{j}}\right\} \mid e_{\ell}=\left\{v_{i}, v_{j}\right\} \text { for some } e_{\ell} \in E(G) \text { and } v_{i} \in V^{\prime}\right\} \cup \\
& \left\{\left\{e_{\ell}, e_{\ell}^{v_{i}}\right\},\left\{f_{\ell}, e^{v_{j}}\right\} \mid e_{\ell}=\left\{v_{i}, v_{j}\right\} \text { for some } e_{\ell} \in E(G) \text { and }\left\{v_{i}, v_{j}\right\} \cap V^{\prime}=\emptyset \text { and } i<j\right\} .
\end{aligned}
$$

Note that the partners of $S$ and $T$ can be of arbitrary order, and that the partners of the agents $e_{\ell}$ and $f_{\ell}$ for which none of the endpoints of the corresponding edge $e_{\ell}$ are in the independent set $S$ can also be swapped. We fix this order for the sake of the simplicity of the reasoning.

One can verify that $M$ is stable in the original profile $P$. To see why it remains stable in every profile, denoted as $P^{\prime}$, that differs from the original one by at most one swap, we observe the following.

1. No agent $a_{j}$ from $A$ is involved in a blocking pair because for each agent $y \neq b_{j}=M\left(a_{j}\right)$ other than $a_{j}$ 's partner $b_{j}$ it holds that if $a_{j}$ shall prefer $y$ to $b_{j}$ in $P^{\prime}$, then this agent $y$ must be $b_{1-j}$. However, agent $b_{1-j}$ will still prefer her partner $a_{1-j}$ to $a_{j}$ because $P$ and $P^{\prime}$ differ by only one swap.

2. Analogously, no agent $b_{j}$ from $B$ is involved in a blocking pair.

3. No agent $z$ from $S \cup T$ is involved in a blocking pair because of the following. The partner of $z$ is an agent from $V$. For each agent $y \neq M(z)$ other than $z$ 's partner $M(z)$ if agents $z$ and $y$ would form a blocking pair in $P^{\prime}$ then $z$ would have preferred $y$ to an agent from $M(z)$. To achieve this, however, we need at least $|V|-1$ swaps, which is more than two.

4. Analogously, no agent $e_{\ell}$ from $E$ is involved in a blocking pair because of the following. The partner $M\left(e_{\ell}\right)$ of $e_{\ell}$ is an agent from $\left\{e_{\ell}^{v_{i}}, e_{\ell}^{v_{j}}\right\}$ with $e_{\ell}=\left\{v_{i}, v_{j}\right\}$. For each agent $y \neq M\left(e_{\ell}\right)$ other than $e_{\ell}$ 's partner $M\left(e_{\ell}\right)$ if $e_{\ell}$ would form with $y$ a blocking pair in $P^{\prime}$ then she must have preferred $y$ to her partner $M\left(e_{\ell}\right)$. To achieve this, we would need at least 2 swaps.

5. No agent $f_{\ell}$ from $F$ is involved in a blocking pair because of the following. The partner of $f_{\ell}$ is an agent from $\left\{e_{\ell}^{v_{i}}, e_{\ell}^{v_{j}}\right\}$ with $e=\left\{v_{i}, v_{j}\right\}$. For each agent $y \neq M\left(f_{\ell}\right)$ other than $f_{\ell}$ 's partner $M\left(f_{\ell}\right)$ if agent $f_{\ell}$ would form with $y$ a blocking pair of $M$, then she must have preferred $y$ to $M\left(f_{\ell}\right)$. By the preference list of $f_{\ell}$ and by the definition of $M$ this agent $y$ must be an agent $e_{\ell}^{v}$ with $v \in e$ and $M\left(e_{\ell}^{v}\right)=e_{\ell}$. However, agent $y$ will still prefer her partner $e_{\ell}$ to $f_{\ell}$ as the swap distance between $e_{\ell}$ and $f_{\ell}$ in the initial preference list of $e_{\ell}^{v}$ is two.

6. Agents $v_{i}$ and $e_{\ell}^{v_{j}}$ with $v_{i} \neq v_{j}$ cannot form a blocking pair of $M$ in $P^{\prime}$ because the swap distance between $e_{\ell}^{v_{j}}$ and $M\left(v_{i}\right) \in S \cup T$ in the initial preference list of $v_{i}$ is at least $|S|$, which is more than one.

Thus, the only possible blocking pairs would involve $v_{i}$ and $e_{\ell}^{v_{i}}$ with $v_{i} \in e_{\ell}$ for some $e_{\ell} \in E(G)$. Suppose, for the sake of contradiction, that $\left\{v_{i}, e_{\ell}^{v_{i}}\right\}$ is blocking $M$ in $P^{\prime}$. This implies that $M\left(v_{i}\right) \in$ $S$ and $M\left(e_{\ell}^{v_{i}}\right)=f_{\ell}$. However, by construction, $M\left(v_{i}\right) \in S$ implies that $v_{i} \in V^{\prime}$ and $M\left(e_{\ell}^{v_{i}}\right)=f_{\ell}$ implies that $v_{i} \notin V^{\prime}$-a contradiction.

For the "if" direction, let $M$ be a stable matching that is stable in every profile that differs from the original one by one swap. We claim that $V^{\prime}=\left\{v_{i} \mid M\left(v_{i}\right) \in S\right\}$ is an independent set of size $k$. Obviously $V^{\prime}$ has $k$ vertices by our observation above that every agent $v_{i}$ is matched to an agent that is either from $T$ or from $S$, and $|S|=k$. To show that $V^{\prime}$ is an independent set, suppose 
for the sake of contradiction that $V^{\prime}$ contains two adjacent vertices $v_{i}$ and $v_{j}$ and let $e_{\ell}=\left\{v_{i}, v_{j}\right\}$ be the incident edge. This means that $M\left(v_{i}\right), M\left(v_{j}\right) \in S$. Then, consider the profile $P^{\prime}$ that differs from $P$ by one swap in the preference list of $e_{\ell}^{v_{i}}$, depicted as follows:

$$
e_{\ell}^{v_{i}}: e_{\ell} \succ a_{0} \succ v_{i} \succ f_{\ell}
$$

Since $v_{i}$ prefers agent $e_{\ell}^{v_{i}}$ to its partner which is from $S$, the stability of $M$ implies that $M\left(e_{\ell}^{v_{i}}\right)=e_{\ell}$; recall that $M\left(e_{\ell}^{v_{i}}\right)$ cannot be matched to $a_{0}$ as reasoned before. Consequently, $M\left(e_{\ell}^{v_{j}}\right)=f_{\ell}$. However, consider the profile $P^{\prime \prime}$ that differs from $P$ by one swap in the preference list of $e_{\ell}^{v_{j}}$, depicted as follows:

$$
e_{\ell}^{v_{j}}: e_{\ell} \succ a_{0} \succ v_{j} \succ f_{\ell} .
$$

Since $v_{i}$ prefers agent $e_{\ell}^{v_{j}}$ to its partner which is from $S$, it follows that $M$ is not stable in $P^{\prime \prime}$ as we have just reasoned that $M\left(e_{\ell}^{v_{j}}\right)=f_{\ell}$ but $v_{j}$ prefers $v_{j}$ to $f_{\ell}$ in $P^{\prime \prime}$-a contradiction.

\section{$5 \quad$ Nearly Stable Matchings}

We now present our results on the complexity of finding nearly stable matchings which are perfect or within a given egalitarian cost bound. We start in Section 5.1 by observing that all four problems variants of near stability are NP-hard. Indeed, we provide a stronger result, which says that under the standard complexity theory assumption $\mathrm{P} \neq \mathrm{NP}$ the minimization variants of all considered problems do not admit a polynomial-time polynomial-factor approximation algorithm. In Section 5.2 we study the influence of the number of allowed swaps on the complexity of the problem variants.

\subsection{Classical and Approximation Hardness}

To show hardness, we will focus on the so-called gap variants of our problems, and prove that these gap variants are NP-hard. These gap problems can be solved by the corresponding approximation algorithms so that their NP-hardness will rule out polynomial-time approximation algorithms for our problem. Loosely speaking, an $\alpha$-gap variant of some minimization problem $\mathcal{Q}$ has, as input, a specific instance $I$ of $\mathcal{Q}$ and a cost upper bound $q \in \mathbb{N}$ and asks whether 1 . $I$ admits a solution of cost at most $q$, or 2 . each solution for $I$ has cost at least $\alpha \cdot q$ (without requirement on the answer when the optimum solution is in the "gap" interval $(q, \alpha \cdot q))$. Note that, to decide between these two options we can use a factor- $\alpha$ approximation algorithm (if it exists), an algorithm that is guaranteed to find a solution of cost at most $\alpha \cdot$ opt where opt is the minimum cost. Hence, if the $\alpha$-gap problem is NP-hard, a polynomial-time factor- $\alpha$ approximation algorithm implies $\mathrm{P}=\mathrm{NP}$.

To make the presentation easier, we use the following decision-focused definition of approximation algorithms, which only solve the gap variant of an optimization problem. By the reasoning above, ruling out the existence of such approximation algorithms also rules out the existence the standard form approximation algorithms which produce solutions.

Definition 5.1. Let poly: $\mathbb{N} \rightarrow \mathbb{N}$ be a polynomial whose domains and co-domains are on the positive integers. An algorithm $\mathcal{A}$ is a polynomial-time and poly-approximation algorithm for Globally Nearly Stable Perfect Matching (Global-Near+Perf) if for each preference

profile $P$ and each positive integer $\mathrm{d}_{\mathrm{G}} \in \mathbb{N}$, the algorithm $\mathcal{A}$ runs in time $|P|^{O(1)}$ and satisfies 
the following: (1) if $P$ admits a globally $\mathrm{d}_{\mathrm{G}}$-nearly stable and perfect matching, then $\mathcal{A}$ returns "yes", and (2) if $P$ admits no globally poly $\left(\mathrm{d}_{\mathrm{G}}\right) \cdot \mathrm{d}_{\mathrm{G}}$-nearly stable and perfect matching, then $\mathcal{A}$ returns " $n o$ ".

If such an algorithm exists, then we also say that GLOBAL-NEAR+PERF admits a polynomialtime and polynomial-factor approximation algorithm.

An approximation algorithm for Locally Nearly Stable Perfect Matching (LocalNEAR+PERF) is defined analogously. For the variant where an additional objective is to achieve a given egalitarian cost, we use an even weaker notion that allows for bi-criteria approximation.

Definition 5.2. Let poly ${ }_{1}$, poly $y_{2}: \mathbb{N} \rightarrow \mathbb{N}$ be two polynomials whose domains and co-domains are on the positive integers. An algorithm $\mathcal{A}$ is a polynomial-time and (poly ${ }_{1}$, poly plapproximation algorithm for Globally Nearly Stable Egalitarian Matching (Global-Near+Egal) if for each preference profile $P$ and each positive integer $\mathrm{d}_{\mathrm{G}} \in \mathbb{N}$, the algorithm $\mathcal{A}$ runs in time $|P|^{O(1)}$ and satisfies the following: (1) if $P$ admits a globally $\mathrm{d}_{\mathrm{G}}$-nearly stable matching with egalitarian cost at most $\eta$, then $\mathcal{A}$ returns "yes", and (2) if $P$ admits no globally poly $_{1}\left(\mathrm{~d}_{\mathrm{G}}\right) \cdot \mathrm{d}_{\mathrm{G}}$-nearly stable matching with egalitarian cost at most poly $_{2}(\eta) \cdot \eta$, then $\mathcal{A}$ returns "no".

If such an algorithm exists, then we also say that GLOBAL-NEAR+EGAL admits a polynomialtime and polynomial-factor approximation algorithm.

Here, again an approximation algorithm for Locally NEARly Stable EGalitarian MatchING (LOCAL-NEAR+EGAL) is defined analogously.

Theorem 5.3. For each $\Pi \in$ \{Global-Near+Egal, Global-Near+Perf, LocalNEAR+PERF, LocAL-NEAR+EGAL $\}$, $\Pi$ is NP-hard, and does not admit a polynomial-time polynomial-factor approximation algorithms, unless $P=N P$. For LOCAL-NEAR+PERF and LOCAL-NEAR+EGAL, the statement holds even if $\mathrm{d}_{\mathrm{L}}=1$.

Proof. The NP-hardness will follow from the inapproximability results by setting the corresponding approximation factors to 1 . Thus, we only need to show the inapproximability results, which are based on the same basic construction. We first give the details of the construction. Then, we prove that, on the instances resulting from the construction, approximability of GLOBAL-NEAR+PERF, Global-Near+Egal, Local-Near+Perf, or Local-Near+Egal implies polynomial-time solvability of all NP-complete problems.

Let poly , poly $_{2}: \mathbb{N} \rightarrow \mathbb{N}$ be two arbitrary polynomials. We will show non-existence of any polynomial-time and poly-approximation algorithm, using a reduction which introduces a gap in the near stability between an optimally nearly stable solution and any other nearly stable solution.

We reduce from InDEPENDENT SET, which has, as input, an undirected graph $G$ with vertex set $V(G)$ and edge set $E(G)$ and a positive integer $k \in \mathbb{N}$, and asks whether $G$ contains an independent set of size $k$, i.e. a $k$-vertex subset of $V^{\prime} \subseteq V(G)$ of pairwise non-adjacent vertices. Let $I=(G, k)$ be an instance of Independent Set. Let $V(G)=\left\{v_{1}, \ldots, v_{n}\right\}$ and $E(G)=\left\{e_{1}, \ldots, e_{m}\right\}$ denote the set of vertices and the set of edges in $G$, respectively. We interpret the edges as twoelement subsets of $V(G)$. For each vertex $v_{i} \in V$, by $E\left(v_{i}\right)$ we denote the set of edges incident with vertex $v_{i}$ in $G$.

From $G$ we will construct a preference profile $P$. The lower thresholds for the gap problems we are constructing are as follows. We define threshold for the number of swaps for a globally nearly stable matching as $\mathrm{d}_{\mathrm{G}}:=m+n$, the threshold for the number of swaps per agent of a locally nearly stable matching as $d_{L}:=1$, and the threshold for the egalitarian cost of a globally $d_{G}$-nearly stable 
matching as $\eta:=k+\left(\right.$ poly $_{1}\left(\mathrm{~d}_{\mathrm{G}}\right) \cdot \mathrm{d}_{\mathrm{G}}+$ poly $\left._{1}\left(\mathrm{~d}_{\mathrm{L}}\right) \cdot \mathrm{d}_{\mathrm{L}}+2\right) \cdot(3 m+(2 n+k) \cdot k+(2 n-k) \cdot(n-k))$. For ease of notation, let $\mathrm{d}_{\mathrm{G}}^{*}:=\operatorname{poly}_{1}\left(\mathrm{~d}_{\mathrm{G}}\right) \cdot \mathrm{d}_{\mathrm{G}}+\operatorname{poly}_{1}\left(\mathrm{~d}_{\mathrm{L}}\right) \cdot \mathrm{d}_{\mathrm{L}}+1$, and $\eta^{*}:=\operatorname{poly}_{2}(\eta) \cdot \eta+2$.

Construction. We construct a profile $P$ as follows. We introduce the following disjoint sets of agents: $V, T, E, F$ (men); $W, S, R, E_{V}$ (women); and two disjoint sets $A \cup B$ and $C \cup D$ of auxiliary agents. Sets $V$ and $W$ will represent the vertices of $G$, sets $R, S$, and $T$ will force a selection of $k$ vertices, and sets $E, E_{V}$, and $F$ will ensure that the selected vertices are pairwise nonadjacent. The auxiliary agents from $A \cup B$ enforce that only swaps of some specific agents are relevant while the auxiliary agents from $C \cup D$ require that each matching within some appropriate egalitarian cost must be perfect.

The non-auxiliary agents. Specifically, the non-auxiliary sets contain the following agents:

$$
\begin{array}{ll}
V:=\left\{v_{i} \mid v_{i} \in V(G)\right\}, & W:=\left\{w_{i} \mid v_{i} \in V(G)\right\}, \\
T:=\left\{t_{i} \mid v_{i} \in V(G)\right\}, & S:=\left\{s_{i} \mid i \in[k]\right\}, \\
E:=\left\{e_{\ell} \mid e_{\ell} \in E(G)\right\}, & R:=\left\{r_{i} \mid i \in[n-k]\right\}, \\
F:=\left\{e_{\ell}^{v_{i}}, e_{\ell}^{v_{j}} \mid e_{\ell}=\left\{v_{i}, v_{j}\right\} \text { for some edge } e_{\ell} \in E(G)\right\} . &
\end{array}
$$

Note that we use $v_{i}\left(\right.$ resp. $e_{\ell}$ ) for both a vertex and its corresponding vertex agent (resp. an edge and its corresponding edge agent). It will, however, be clear from the context what we are referring to. The preference lists of the above agents are defined as follows (men are placed on the left and women on the right). For the sake of readability the non-auxiliary agents are omitted in each list, and we will describe them in detail later on.

$$
\begin{aligned}
& \forall v_{i} \in V(G): \quad v_{i}: w_{i} \succ\left[\left\{e_{\ell}^{v_{i}} \mid e_{\ell} \in E\left(v_{i}\right)\right\}\right] \succ s_{1} \succ \ldots \succ s_{k}, \quad w_{i}: v_{i} \succ t_{i}, \\
& t_{i}: w_{i} \succ r_{1} \succ \ldots \succ r_{n-k} \text {, } \\
& \forall j \in[n-k]: \quad \quad r_{j}: t_{1} \succ \ldots \succ t_{n}, \\
& \forall j \in[k]: \quad s_{j}: v_{1} \succ \ldots \succ v_{n}, \\
& \forall e_{\ell}=\left\{v_{i}, v_{j}\right\} \in E(G) \text { with } i<j: \quad e_{\ell}: e_{\ell}^{v_{i}} \succ e_{\ell}^{v_{j}}, \quad e_{\ell}^{v_{i}}: e_{\ell} \succ v_{i} \succ f_{\ell}, \\
& f_{\ell}: e_{\ell}^{v_{i}} \succ e_{\ell}^{v_{j}}, \quad e_{\ell}^{v_{j}}: e_{\ell} \succ v_{j} \succ f_{\ell} \text {. }
\end{aligned}
$$

The type-one auxiliary agents $\boldsymbol{A} \cup \boldsymbol{B}$. These agents ensure that only the swaps from the agents of $W \cup E$ are relevant. We say that the auxiliary agents in $A \cup B$ are of type one. For each agent $x$ from $V \cup T \cup F \cup S \cup R \cup E_{V}$ and for each two consecutive agents $y_{1}$ and $y_{2}$ in $x$ 's preference list (as described above), we introduce $\mathrm{d}_{\mathrm{G}}^{*}$ auxiliary agents $a_{x}^{1}\left(y_{1}, y_{2}\right), \ldots, a_{x}^{\mathrm{d}_{\mathrm{G}}^{*}}\left(y_{1}, y_{2}\right)$ to $A$ and $\mathrm{d}_{\mathrm{G}}^{*}$ auxiliary agents $b_{x}^{1}\left(y_{1}, y_{2}\right), \ldots, b_{x}^{\mathrm{d}_{\mathrm{G}}^{*}}\left(y_{1}, y_{2}\right)$ to $B$ with the following preference lists: (i) If $x \in V \cup T \cup F$, then for all $i \in\left[\mathrm{d}_{\mathrm{G}}^{*}\right]$ let the preference lists of $a_{x}^{i}\left(y_{1}, y_{2}\right)$ and $b_{x}^{i}\left(y_{1}, y_{2}\right)$ be $a_{x}^{i}\left(y_{1}, y_{2}\right)$ and $a_{x}^{i}\left(y_{1}, y_{2}\right) \succ x$, respectively, and add all $\mathrm{d}_{\mathrm{G}}^{*}$ auxiliary agents $b_{x}^{i}\left(y_{1}, y_{2}\right)$ between agents $y_{1}$ and $y_{2}$ in the preference list of $x$. (ii) Otherwise, meaning that $x \in R \cup S \cup E_{V}$, then for all $i \in\left[\mathrm{d}_{\mathrm{G}}^{*}\right.$ let the preference lists of $a_{x}^{i}\left(y_{1}, y_{2}\right)$ and $b_{x}^{i}\left(y_{1}, y_{2}\right)$ be $a_{x}^{i}\left(y_{1}, y_{2}\right) \succ x$ and $a_{x}^{i}\left(y_{1}, y_{2}\right)$, respectively, and add all $\mathrm{d}_{\mathrm{G}}^{*}$ auxiliary agents $a_{x}^{i}\left(y_{1}, y_{2}\right)$ between agents $y_{1}$ and $y_{2}$ in the preference list of $x$. In total, we have $|A|=|B|=\mathrm{d}_{\mathrm{G}}^{*} \cdot\left(\sum_{i=1}^{n}\left(\left|E\left(v_{i}\right)\right|+k\right)+n \cdot(n-k)+m+(n-k) \cdot(n-1)+k \cdot(n-1)+2 \cdot 2 m\right)=$ $\mathrm{d}_{\mathrm{G}}^{*} \cdot\left(2 n^{2}-n+7 m\right)$.

Type-two auxiliary agents $\boldsymbol{C} \cup \boldsymbol{D}$. To enforce that every matching within egalitarian cost $\operatorname{poly}_{2}(\eta) \cdot \eta$ must be perfect, we introduce type-two auxiliary agents and append them to the preference list of each non-auxiliary agent and each type-one auxiliary agent. Formally, for each agent $x \in V \cup T \cup E \cup F \cup A \cup W \cup S \cup R \cup E_{V} \cup B$, we introduce $\eta^{*}$ auxiliary agents $C_{x}:=\left\{c_{x}^{1}\right.$, $\left.\ldots, c_{x}^{\eta^{*}}\right\}$ and add them to $C$, and $\eta^{*}$ auxiliary agents $D_{x}\left\{d_{x}^{1}, \ldots, d_{x}^{\eta^{*}}\right\}$ and add them to $D$; recall that $\eta^{*}=\operatorname{poly}_{2}(\eta) \cdot \eta+2$. The preference lists of these agents are as follows: (i) If $x \in V \cup T \cup E \cup F \cup A$, then 
for all $i \in\left[\eta^{*}\right]$ let the preference lists of $c_{x}^{i}$ and $d_{x}^{i}$ be $d_{x}^{i} \succ\left[D_{x} \backslash\left\{d_{x}^{i}\right\}\right]$ and $c_{x}^{i} \succ\left[C_{x} \backslash\left\{c_{x}^{i}\right\}\right] \succ x$, respectively, and append all $\eta^{*}$ auxiliary agents $d_{x}^{i}$ to the end of the preference list of $x$. (ii) Otherwise, that is, $x \in W \cup S \cup R \cup E_{V} \cup B$, then for all $i \in\left[\eta^{*}\right]$ let the preference lists of $c_{x}^{i}$ and $d_{x}^{i}$ be $d_{x}^{i} \succ\left[D_{x} \backslash\left\{d_{x}^{i}\right\}\right] \succ$ $x$ and $c_{x}^{i} \succ\left[C_{x} \backslash\left\{c_{x}^{i}\right\}\right]$, respectively, and append all $\eta^{*}$ auxiliary agents $c_{x}^{i}$ to the end of the preference list of $x$. In total, we have $|C|=|D|=\eta^{*} \cdot\left(|V|+|T|+|E|+|F|+|A|+|W|+|R|+|S|+\left|E_{V}\right|+|B|\right)$. Observe that every matching with egalitarian cost at most poly ${ }_{2}(\eta) \cdot \eta$ must assign to every type-two auxiliary agent a partner that is also of type two, as otherwise the egalitarian cost induced by such two agents would be at least $\eta^{*}-1=\operatorname{poly}_{2}(\eta) \cdot \eta+1>\operatorname{poly}_{2}(\eta) \cdot \eta$.

This completes the construction of the profile $P$. Clearly, it can be constructed in polynomial time.

Correctness of the construction. In the following, we show that the existence of any polynomialtime poly ${ }_{1}$-factor approximation algorithm for GLOBAL-NEAR+PERF or LOCAL-NEAR+PERF or any polynomial-time $\left(\right.$ poly $_{1}$, poly p $_{2}$-factor approximation algorithm for GLOBAL-NEAR+EGAL or LOCAL-NEAR+EgAL implies P = NP. More precisely, we claim the following.

Claim 2. (1) If $G$ admits a k-vertex independent set, then $P$ admits a globally $\mathrm{d}_{\mathrm{G}}$-nearly stable and perfect matching, which is also locally $\mathrm{d}_{\mathrm{L}}$-nearly stable and has egalitarian cost at most $\eta$.

(2) If $P$ admits a globally poly ${ }_{1}\left(\mathrm{~d}_{\mathrm{G}}\right) \cdot \mathrm{d}_{\mathrm{G}}$-nearly stable and perfect matching, then $G$ admits a $k$-vertex independent set.

(3) If $P$ admits a locally poly ${ }_{1}\left(\mathrm{~d}_{\mathrm{L}}\right) \cdot \mathrm{d}_{\mathrm{L}}$-nearly stable and perfect matching, then $G$ admits a $k$-vertex independent set.

Proof. To show the first statement, assume that $V^{\star} \subseteq V$ is a $k$-vertex independent set of $G$. Construct a perfect matching $M$ for $P$ as follows. Let $i_{1}<i_{2}<\ldots<i_{k}$ be the indices of the vertices in $V^{\star}$, that is, for each $z \in[k]$ we have $v_{i_{z}} \in V^{\star}$. Similarly, let $j_{1}<j_{2}<\ldots<j_{n-k}$ be the indices of the vertices in $V \backslash V^{\star}$. Matching $M$ contains the following pairs.

(i) For each $z \in[k]$, match $\left\{v_{i_{z}}, s_{z}\right\} \in M$ and $\left\{t_{i_{z}}, w_{i_{z}}\right\} \in M$.

By the construction of the preference lists, the egalitarian cost of the pair $\left\{v_{i_{z}}, s_{z}\right\}$ is at most $\left(\mathrm{d}_{\mathrm{G}}^{*}+1\right) \cdot(n+k+k)$; recall that, for each agent $x$ who is not an auxiliary agent and who is not in $W \cup E$, we have placed exactly $\left(\mathrm{d}_{\mathrm{G}}^{*}+1\right)$ type-one auxiliary agents between each pair of non-auxiliary agents in $x$ 's preference list. The egalitarian cost of the pair $\left\{t_{i_{z}}, w_{i_{z}}\right\}$ is one. In total, these $2 \cdot k$ pairs contribute at most $k+\left(\mathrm{d}_{\mathrm{G}}^{*}+1\right) \cdot(n+2 \cdot k) \cdot k$ units to the egalitarian cost.

(ii) For each $z \in[n-k]$, match $\left\{v_{j_{z}}, w_{j_{z}}\right\} \in M$ and $\left\{t_{j_{z}}, r_{z}\right\} \in M$.

The egalitarian cost of the pair $\left\{v_{j_{z}}, w_{j_{z}}\right\}$ is zero, while the egalitarian cost of the pair $\left\{t_{j_{z}}, r_{z}\right\}$ is at most $\left(\mathrm{d}_{\mathrm{G}}^{*}+1\right) \cdot(n-k+n)$. In total, these $2 \cdot(n-k)$ pairs contribute at most $\left(\mathrm{d}_{\mathrm{G}}^{*}+1\right) \cdot(n-k+n) \cdot(n-k)$ units to the egalitarian cost.

(iii) Further, for each edge $e_{\ell} \in E(G)$, choose an endpoint from $e_{\ell} \cap V^{\star}$ or an arbitrary endpoint of $e_{\ell}$ if $e_{\ell} \cap V^{\star}=\emptyset$. Say we have picked an endpoint with index $i$. Then, match $\left\{e_{\ell}, e_{\ell}^{v_{i}}\right\},\left\{f_{\ell}, e_{\ell}^{v_{j}}\right\} \in M$, where $j$ is the index of the other endpoint of $e_{\ell}$, different from $i$.

The egalitarian cost of these two pairs is at most $\left(\mathrm{d}_{\mathrm{G}}^{*}+1\right) \cdot 3$.

(iv) Finally, for each type-one auxiliary agent $a_{x}^{z}\left(y_{1}, y_{2}\right) \in A$, match her with its counter-part from $B$, that is, match $\left\{a_{x}^{z}\left(y_{1}, y_{2}\right), b_{x}^{z}\left(y_{1}, y_{2}\right)\right\} \in M$. For each type-two auxiliary agent $c_{x}^{z} \in C$, match it with its counter-part from $D$, that is, match her $\left\{c_{x}^{z}, d_{x}^{z}\right\} \in M$.

There is no egalitarian cost for these pairs.

This concludes the definition of $M$, which is clearly a perfect matching. One can verify that the egalitarian cost of $M$ is at most $k+\left(\mathrm{d}_{\mathrm{G}}^{*}+1\right) \cdot((n+2 \cdot k) \cdot k+(2 n-k) \cdot(n-k)+3 m)=\eta$ 
It remains to show that $M$ is globally $\mathrm{d}_{\mathrm{G}}$-nearly stable and locally $\mathrm{d}_{\mathrm{L}}$-nearly stable. We claim that after performing the following swaps, indeed, $M$ is stable: For each edge agent $e_{\ell} \in E$ let $e_{\ell}^{v_{i}}$ and $e_{\ell}^{v_{j}}$ be the two vertex agents in its preference lists with $i<j$, if $M\left(e_{\ell}\right)=e_{\ell}^{v_{j}}$, then swap the order of these two agents $e_{\ell}^{v_{i}}$ and $e_{\ell}^{v_{j}}$ in $e_{\ell}$ 's preference list; recall that there are no other agents between these two agents. For each agent $w_{i}$ which is not matched to $v_{i}$, swap $v_{i}$ with $t_{i}$ in $w_{i}$ 's preference list; note that there are exactly $k$ such agents. Clearly, each agent has performed at most $1=\mathrm{d}_{\mathrm{L}}$ swap and the total number of performed swaps is at most $m+k \leq \mathrm{d}_{\mathrm{G}}$. Denote by $P^{\prime}$ the profile that results from these swaps.

Next, we show that $M$ is stable for $P^{\prime}$. Clearly, each auxiliary agent receives its most preferred agent, and, hence, no auxiliary agent can be involved in a blocking pair. Similarly, each agent $w_{i} \in W$ receives its most preferred agent (after the $k$ swaps performed on $W$ ), and, hence, no agent from $W$ can be involved in a blocking pair. Since each edge agent $e_{\ell}$ is matched to its most preferred agent, no blocking pair can involve any edge agent $e_{\ell}$. Since the partner $M\left(e_{\ell}\right)$ of each edge agent $e_{\ell}$ already obtains its most preferred agent, no blocking pair can involve any agent $M\left(e_{\ell}\right)$. Further, since these agents, $M\left(e_{\ell}\right)$, are the only agents which may be preferred by any agent $f_{\ell}$ to $M\left(f_{\ell}\right)$, no blocking pair can involve any $f_{\ell}$; recall that we have just reasoned that no auxiliary agent is involved in a blocking pair. Furthermore, each vertex agent $v_{i}$ whose corresponding vertex does not belong to the independent set, i.e. $v_{i} \in V \backslash V^{\star}$ is matched to its most preferred agent. Similarly, each agent $t_{z}$ with $M\left(t_{z}\right)=w_{z}$ cannot be involved in a blocking pair because she already obtains its most preferred agent.

A potential blocking pair must hence involve an agent from $\left\{t_{j_{z}} \mid v_{j_{z}} \in V \backslash V^{\star}\right\} \cup V^{\star}$.

Consider an agent $t_{j_{z}}$ with $v_{j_{z}} \in V \backslash V^{\star}$. By the construction of matching $M$, it follows that $M\left(t_{j_{z}}\right)=r_{z}$. Since neither $w_{j_{z}}$ nor any auxiliary agent can be involved in a blocking pair, by the preference list of $t_{j_{z}}$ it follows that $t_{j_{z}}$ could only form a blocking pair with an agent $r_{z^{\prime}}$ such that $z<z^{\prime}$. However, this agent $r_{z^{\prime}}$ prefers its partner $M\left(r_{z^{\prime}}\right)=t_{j_{z^{\prime}}}$ to $t_{j_{z}}$. Hence, no agent $t_{j_{z}}$ can be involved in a blocking pair.

Consider an agent $v_{i_{z}}$ which corresponds to a vertex from the independent set $V^{\star}$. By the construction of matching $M$, it follows that $M\left(v_{i_{z}}\right)=s_{z}$. By our reasoning above, $w_{i_{z}}$ will not form a blocking pair with $v_{i_{z}}$ as it already obtains its most preferred partner. Agent $v_{i_{z}}$ prefers agent $s_{z^{\prime}}$ to its partner $s_{z}$ only if $z^{\prime}<z$. However, for each $z^{\prime}<z$, agent $s_{z^{\prime}}$ prefers its partner $M\left(s_{z^{\prime}}\right)=v_{i_{z^{\prime}}}$ to agent $v_{i_{z}}$ (recall that $i_{z^{\prime}}<i_{z}$ ). Thus, no agent from $S$ will form with $v_{i_{z}}$ a blocking pair. Any blocking pair must thus be of the form $\left\{v_{i}, e_{\ell}^{v_{i}}\right\}$ where $v_{i} \in V^{\star}$ and $e_{\ell}^{v_{i}} \in E\left(v_{i}\right)$. However, since $v_{i} \in V^{\star}$, for each of its incident edges, say $e_{\ell}$, we have matched $e_{\ell}^{v_{i}}$ to its most preferred agent $e_{\ell}$. Thus, indeed, there is no blocking pair, showing that $M$ is globally $\mathrm{d}_{\mathrm{G}}$-nearly stable and locally $\mathrm{d}_{\mathrm{L}}$-nearly stable.

For the second statement of Claim 2 , assume that $M$ is a globally poly ${ }_{1}\left(\mathrm{~d}_{\mathrm{G}}\right) \cdot \mathrm{d}_{\mathrm{G}}$-nearly stable and perfect matching for $P$ and let $P^{\prime}$ be a profile that results from $P$ by making at most poly $_{1}\left(\mathrm{~d}_{\mathrm{G}}\right) \cdot \mathrm{d}_{\mathrm{G}}$ swaps such that $M$ is stable in $P^{\prime}$.

Recall that for each agent $y$ which is either non-auxiliary or an auxiliary agent of type one we have introduced $2 \cdot \eta^{*}$ type-two auxiliary agents, contained in $C_{y}$ and in $D_{y}$. Observe that either $C_{y}$ or $D_{y}$ finds only the agents from the other set acceptable. Hence, by the perfectness of $M$, the partners of all agents from $C_{y}$ are exactly the agents from $D_{y}$. Consequently, we can ignore all type-two auxiliary agents in the preference lists of the remaining agents. In particular, for each pair of typeone auxiliary agents $a_{x}^{z}\left(y_{1}, y_{2}\right)$ and $b_{x}^{z}\left(y_{1}, y_{2}\right)$, one of them finds only the other agent acceptable (ignoring the type-two auxiliary agents). Again, by the perfectness of $M$, we infer that each $a_{x}^{z}\left(y_{1}, y_{2}\right)$ is matched to its counter-part $b_{x}^{z}\left(y_{1}, y_{2}\right)$. Hence, from now on, when discussing the partners of a 
non-auxiliary agent, we only need to consider the non-auxiliary agents in its preference list.

Recall that there are at least $\mathrm{d}_{\mathrm{G}}^{*}$ type-one auxiliary agents between each non-auxiliary agents in the preference list of each agent from $V \cup R \cup S \cup T \cup E_{v} \cup F$. By the fact that $\mathrm{d}_{\mathrm{G}}^{*}=$ poly $_{1}\left(\mathrm{~d}_{\mathrm{G}}\right) \cdot \mathrm{d}_{\mathrm{G}}+$ poly $_{1}\left(\mathrm{~d}_{\mathrm{L}}\right) \cdot \mathrm{d}_{\mathrm{L}}+1$, it is impossible to perform poly ${ }_{1}\left(\mathrm{~d}_{\mathrm{G}}\right) \cdot \mathrm{d}_{\mathrm{G}}$ swaps so as to switch the positions of two non-auxiliary agents in the preference list of an agent from $V \cup R \cup S \cup T \cup E_{V} \cup F$. Thus, the only swaps performed to obtain $P^{\prime}$ are without loss of generality in the preference lists of agents in $E \cup W$ and are only within the non-auxiliary agents.

Let $V^{\prime}=\left\{v_{i} \in V(G) \mid M\left(v_{i}\right) \in S\right\}$. We claim that $V^{\prime}$ is a $k$-vertex independent set in $G$. First, $V^{\prime}$ has cardinality $k$ because $M$ is perfect and the only remaining acceptable partners to every agent in $S$ are those in $V$. Suppose, for the sake of contradiction, that $V^{\prime}$ contains two adjacent vertices $v_{i}$ and $v_{j}$ and let $e_{\ell}=\left\{v_{i}, v_{j}\right\}$ be their incident edge. By the definition of $V^{\prime}$, it follows that both $v_{i}$ and $v_{j}$ are assigned partners from $S$. Since $v_{i}$ prefers $e_{\ell}^{v_{i}}$ to every agent from $S$ and since $e_{\ell}^{v_{i}}$ prefers only $e_{\ell}$ to $v_{i}$, by the stability of $M$ in $P^{\prime}$, it follows that $M\left(e_{\ell}^{v_{i}}\right)=e_{\ell}$; recall that no swaps are performed in between any two non-auxiliary agents in the preference lists of the agents from $V \cup E_{V}$. Analogously, it must hold that $M\left(e_{\ell}^{v_{j}}\right)=e_{\ell}$ - a contradiction to $M$ being a matching.

The reasoning for the third statement is analogous to what we have done for the second statement. Instead of arguing about the total number of swaps, we only need to argue that the number of swaps changed per agent is poly ${ }_{1}\left(d_{L}\right) \cdot d_{L}$ which is strictly smaller than by $d_{G}^{*}$. Thus, it is still impossible to change the positions of two non-auxiliary agents in any the preference list of any non-auxiliary agent from $V \cup R \cup S \cup T \cup E_{v} \cup F$.

$($ of Claim 2) $\diamond$

The next claim establishes a close connection between the egalitarian cost and the perfectness of a matching.

Claim 3. If $M$ is a matching with egalitarian cost of at most poly $_{2}(\eta) \cdot \eta$, then this matching must be perfect.

Proof. Assume that $M$ has egalitarian cost at most poly ${ }_{2}(\eta) \cdot \eta$. It is straight-forward to see that this $M$ must be perfect as otherwise the cost of leaving one agent unmatched is equal to the length of this agent's preference list, exceeding the budget poly ${ }_{2}(\eta) \cdot \eta$ because the length of each agent's preference list is at least $\eta^{*}>\operatorname{poly}_{2}(\eta) \cdot \eta+1$.

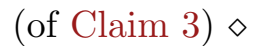

Now, we continue with our correctness proof.

Inapproximability of GLOBAL-NEAR+PERF. Suppose, for the sake of contradiction, that there exists a poly ${ }_{1}$-approximation algorithm $\mathcal{A}$ for GLOBAL-NEAR+PERF, running in polynomial-time. Then, we can use algorithm $\mathcal{A}$ to decide IndEPENDEnT SET in polynomial time, showing $\mathrm{P}=\mathrm{NP}$. Given an arbitrary instance $I=(G, k)$ of IndePEndent SET, we construct profile $P$ and define $\mathrm{d}_{\mathrm{G}}$ as described above and let $\mathcal{A}$ run on $\left(P, \mathrm{~d}_{\mathrm{G}}\right)$. If $I$ is a yes-instance, then by the first implication of Claim 2, it follows that $P$ admits a globally $\mathrm{d}_{\mathrm{G}}$-nearly stable and perfect matching, and by Definition 5.1, $\mathcal{A}$ return "yes". If $I$ is a no-instance, then by the contrapositive of the second implication of Claim 2 , it follows that $P$ does not admit a globally poly ${ }_{1}\left(\mathrm{~d}_{\mathrm{G}}\right) \cdot \mathrm{d}_{\mathrm{G}}$-nearly stable and perfect matching. By Definition 5.1, $\mathcal{A}$ returns "no".

Inapproximability of GLOBAL-NEAR+EGAL. Again, suppose, for the sake of contradiction, that there exists a ( poly $_{1}$, poly ) $_{2}$-approximation algorithm $\mathcal{A}$ for GLOBAL-NEAR+EGAL, running in polynomial-time. Then, we can use algorithm $\mathcal{A}$ to decide InDEPENDENT SET in polynomial time, showing $\mathrm{P}=\mathrm{NP}$, as follows. Given an arbitrary instance $I=(G, k)$ of IndePendent SET, we construct profile $P$ and define $\mathrm{d}_{\mathrm{G}}, \mathrm{d}_{\mathrm{L}}$, and $\eta$ as described above and let $\mathcal{A}$ run on $\left(P, \mathrm{~d}_{\mathrm{G}}, \eta\right)$. 
If $I$ is a yes-instance, then by the first implication of Claim 2 it follows that $P$ admits a globally $\mathrm{d}_{\mathrm{G}}$-nearly stable matching with egalitarian cost at most $\eta$, and by Definition $5.2, \mathcal{A}$ return "yes". If $I$ is a no-instance, then by the contrapositive of the second implication of Claim 2 it follows that $P$ does not admit a globally poly ${ }_{1}\left(\mathrm{~d}_{\mathrm{G}}\right) \cdot \mathrm{d}_{\mathrm{G}}$-nearly stable perfect matching. This implies that $P$ does not admit a globally poly ${ }_{1}\left(\mathrm{~d}_{\mathrm{G}}\right) \cdot \mathrm{d}_{\mathrm{G}}$-nearly stable matching with egalitarian cost at most poly $_{2}(\eta) \cdot \eta$ as otherwise, by Claim 3 , we will have a globally poly ${ }_{1}\left(\mathrm{~d}_{\mathrm{G}}\right) \cdot \mathrm{d}_{\mathrm{G}}$-nearly stable and perfect matching for $P$ - a contradiction. By Definition 5.2, $\mathcal{A}$ returns "no".

Inapproximability of LOCAL-NEAR+PERF. Suppose, for the sake of contradiction, that there exists a poly ${ }_{1}$-approximation algorithm $\mathcal{A}$ for LOCAL-NEAR+PERF, running in polynomial-time. Then, we can use algorithm $\mathcal{A}$ to decide IndePEndent Set in polynomial time, showing $\mathrm{P}=\mathrm{NP}$, as follows. Given an arbitrary instance $I=(G, k)$ of IndePEndent Set, we construct profile $P$ and define $\mathrm{d}_{\mathrm{G}}, \mathrm{d}_{\mathrm{L}}$, and $\eta$ as described above and let $\mathcal{A}$ run on $\left(P, \mathrm{~d}_{\mathrm{L}}\right)$. If $I$ is a yes-instance, then by the first implication of Claim $2, P$ also admits a locally $\mathrm{d}_{\mathrm{L}}$-nearly stable and perfect matching. Thus, by Definition 5.1, $\mathcal{A}$ return "yes". If $I$ is a no-instance, then by the contrapositive of the third implication from Claim $2, P$ does not admit a locally poly ${ }_{1}\left(\mathrm{~d}_{\mathrm{L}}\right) \cdot \mathrm{d}_{\mathrm{L}}$-nearly stable perfect matching. By Definition 5.1, $\mathcal{A}$ returns "no".

Inapproximability of LOCAL-NEAR+EGAL. Suppose, for the sake of contradiction, that there exists a ( poly $_{1}$, poly ) $_{2}$-approximation algorithm $\mathcal{A}$ for LOCAL-NEAR+EGAL, running in polynomialtime. Then, we can use algorithm $\mathcal{A}$ to decide IndePendent SET in polynomial time, showing $\mathrm{P}=$ NP as follows. Given an arbitrary instance $I=(G, k)$ of Independent Set, we construct profile $P$ and define $\mathrm{d}_{\mathrm{G}}, \mathrm{d}_{\mathrm{L}}$, and $\eta$ as described above and let $\mathcal{A}$ run on $\left(P, \mathrm{~d}_{\mathrm{L}}, \eta\right)$. If $I$ is a yes-instance, then by the first implication of Claim 2 it follows that $P$ admits a locally $\mathrm{d}_{\mathrm{L}}$-nearly stable matching with egalitarian cost at most $\eta$, and by Definition $5.2, \mathcal{A}$ return "yes". If $I$ is a no-instance, then by the contrapositive of the third implication from Claim 2, $P$ does not admit a locally poly ${ }_{1}\left(\mathrm{~d}_{\mathrm{L}}\right) \cdot \mathrm{d}_{\mathrm{L}}$-nearly stable perfect matching. By the contrapositive of Claim $3, P$ does not admit a locally poly $y_{1}\left(d_{\mathrm{L}}\right) \cdot \mathrm{d}_{\mathrm{L}^{-}}$ nearly stable matching with egalitarian cost at most $\eta$. By Definition $5.2, \mathcal{A}$ returns "no".

\subsection{Parameterized Complexity}

We now investigate the influence of three natural parameters on the complexity of obtaining nearly stable matchings: "total number $\mathrm{d}_{\mathrm{G}}$ of swaps", "number $\mathrm{n}_{\mathrm{u}}$ of initially unmatched agents", and "number $\mathrm{n}_{\mathrm{m}}$ of initially matched agents"; the latter two will be defined below. Note that Theorem 5.3 implies that even only one swap leaves the problems pertaining to locally nearly stable matchings NP-hard. This is different from the globally nearly stable variants, for which simple polynomial-time algorithms for a constant number of swaps exist. However, we show that removing the dependence on the number of swaps in the exponent in the running time is impossible unless $\mathrm{FPT}=\mathrm{W}[1]$.

Proposition 5.4. Global-NeAR+Perf and Global-NeAR+Egal are solvable in $n^{O\left(\mathrm{~d}_{\mathrm{G}}\right)}$ time.

Proof sketch. Iterate over all $\left(\begin{array}{c}n^{2} \\ d_{G}\end{array}\right)$ possibilities for making $d_{G}$ swaps and check for each of the resulting profiles using the well-known polynomial-time algorithms [15, 20] whether it admits a stable matching which is perfect, or satisfies the required bound on the egalitarian cost.

A substantial improvement on the above rather trivial $n^{O\left(\mathrm{~d}_{\mathrm{G}}\right)}$-time algorithm would imply a major breakthrough, as the following theorem shows. 
Theorem 5.5. Global-Near+Perf and Global-Near+Egal are W[1]-hard with respect to the number $\mathrm{d}_{\mathrm{G}}$ of swaps. Moreover, they both do not admit any $n^{o\left(\mathrm{~d}_{\mathrm{G}}\right)}$-time algorithm unless the Exponential Time Hypothesis fails.

Proof. We first show that GLOBAL-NEAR+PERF is $\mathrm{W}[1]$-hard for $\mathrm{d}_{\mathrm{G}}$ and refutes $n^{o\left(\mathrm{~d}_{\mathrm{G}}\right)_{-}}$-running time algorithms. Then, we show how to adapt the proof to show an analogous result for GLOBALNEAR+Egal. To show the results for GLOBAL-NEAR+PERF, we provide a polynomial-time reduction from the $\mathrm{W}[1]$-complete InDEPENDENT SET problem, parameterized by the solution size $k$ [10], and set the parameter to $\mathrm{d}_{\mathrm{G}}=2 k$. Let $(G, k)$ be an instance of InDEPEndEnT SET where we seek for an independent set of size $k$ in the $n$-vertex, $m$-edge graph $G$, with $V(G)=\left\{v_{1}, \ldots, v_{n}\right\}$ and $E(G)=\left\{e_{1}, \ldots, e_{m}\right\}$. We construct a preference profile $P$ with two disjoint sets of agents, $A$ and $B$, each consisting of five groups and a dummy agent: $A:=T \cup V \cup W \cup E \cup E_{Y} \cup\left\{h_{1}\right\}$ and $B:=S \cup X \cup Y \cup F \cup F_{V} \cup\left\{h_{2}\right\}$. Note that we use the symbols $v_{i}$ (resp. $e_{\ell}$ ) for both vertices and agents (resp. for both edges and agents). It will, however, be clear from the context what we are referring to when we use them. The two dummy agents $h_{1}$ and $h_{2}$ are used to make performing some swaps non-beneficial.

Agent sets $\boldsymbol{T}$ and $\boldsymbol{S}$. For each $z \in[k]$, introduce two selection agents $t_{z}$ and $s_{z}$ add them to $T$ and $S$, respectively. These agents will be unmatched in every stable matching of $P$ and matching them will force a selection of $k$ vertices from $G$ into an independent set. Their acceptable agents are a subset of the vertex agents that we introduce as follows.

Agent sets $\boldsymbol{V}, \boldsymbol{W}, \boldsymbol{X}$, and $\boldsymbol{Y}$. For each vertex $v_{i} \in V(G)$, introduce four agents $v_{i}, w_{i}, x_{i}$, and $y_{i}$, and add them to the sets $V, W, X$, and $Y$, respectively. For each $i \in[n]$, these agents will form a path $v_{i}, x_{i}, w_{i}, y_{i}$ in the acceptability graph. The basic idea is that, in the initial profile, every stable matching must match agent $v_{i}$ to agent $x_{i}$ and agent $w_{i}$ to $y_{i}$. As we will see, such matchings are imperfect since the agents from $S$ are unmatched in every stable matching. To obtain a perfect matching, we must match some selection agent $s_{z} \in S$ to some vertex agent $v_{i}$, selecting the corresponding vertex $v_{i}$ into a solution for the input graph. This will incur two swaps to make the resulting matching stable. Below, we introduce edge agents and add them to the preference lists of agents $v_{i}$ and $y_{i}$, in order to ensure that the selected vertices induce an independent set.

Agent sets $\boldsymbol{E}, \boldsymbol{E}_{\boldsymbol{Y}}, \boldsymbol{F}$, and $\boldsymbol{F}_{\boldsymbol{V}}$. For each edge $e_{\ell} \in E(G)$, denote the endpoints of $e_{\ell}$ by $v_{i}$ and $v_{j}$ such that $i<j$. Introduce four edge agents $e_{\ell}, e_{\ell}^{y_{j}}, f_{\ell}$, and $f_{\ell}^{v_{i}}$, and add them to $E, E_{Y}, F$, and $F_{V}$, respectively.

The preference lists of the agents are constructed as follows. Here, for some set $Z$, the notation $[Z]$ means an arbitrary but fixed linear order of $Z$.

\section{Preference lists of the agents.}

$$
\begin{aligned}
& h_{1}: h_{2} \succ[Y], h_{2}: h_{1} \succ[V], \\
& \forall z \in[k], s_{z}: v_{1} \succ \ldots \succ v_{n} . \\
& \quad t_{z}: y_{1} \succ \ldots \succ y_{n}, \\
& \forall i \in[n], x_{i}: v_{i} \succ w_{i}, \\
& v_{i}: x_{i} \succ h_{2} \succ\left[\left\{f_{\ell}^{v_{i}} \mid e_{\ell}=\left\{v_{i}, v_{j}\right\} \in E(G) \text { with } i<j\right\}\right] \succ s_{1} \succ \ldots \succ s_{k}, \\
& w_{i}: y_{i} \succ x_{i}, y_{i}: w_{i} \succ h_{1} \succ\left[\left\{e_{\ell}^{y_{i}} \mid e_{\ell}=\left\{v_{r}, v_{i}\right\} \in E(G) \text { with } r<i\right\}\right] \succ t_{1} \succ \ldots \succ t_{k}, \\
& \forall \ell \in[m] \text { with } e_{\ell}=\left\{v_{i}, v_{j}\right\} \text { and } i<j, \\
& e_{\ell}: f_{\ell}^{v_{i}} \succ f_{\ell}, f_{\ell}: e_{\ell} \succ e_{\ell}^{y_{j}}, \\
& e_{\ell}^{y_{j}}: f_{\ell} \succ y_{j} \succ f_{\ell}^{v_{i}}, f_{\ell}^{v_{i}}: e_{\ell}^{y_{j}} \succ v_{i} \succ e_{\ell} .
\end{aligned}
$$




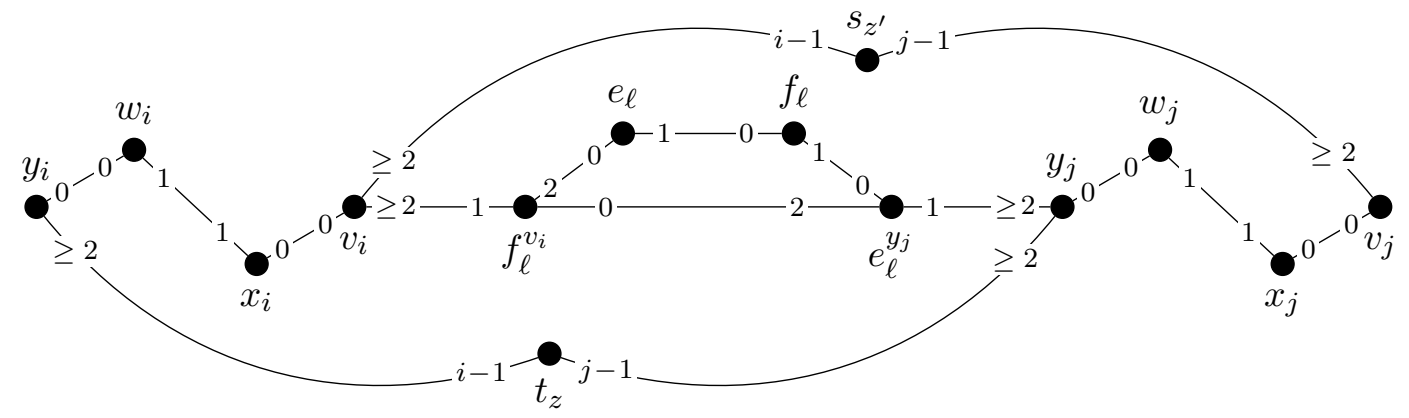

Figure 2: Part of the acceptability graph of the profile constructed in the proof of Theorem 5.5.

Agent sets $\boldsymbol{T}$ and $\boldsymbol{S}$. These agents will be unmatched in every stable matching of $P$ and matching them will force a selection of $k$ vertices from $G$ into an independent set. Their acceptable agents are a subset of the vertex agents that we introduce as follows.

Agent sets $\boldsymbol{V}, \boldsymbol{W}, \boldsymbol{X}$, and $\boldsymbol{Y}$. For each vertex $v_{i} \in V(G)$, introduce four agents $v_{i}, w_{i}, x_{i}$, and $y_{i}$, and add them to the sets $V, W, X$, and $Y$, respectively. For each $i \in[n]$, these agents will form a path $v_{i}, x_{i}, w_{i}, y_{i}$ in the acceptability graph. The basic idea is that, in the initial profile, every stable matching must match agent $v_{i}$ to agent $x_{i}$ and agent $w_{i}$ to $y_{i}$. As we will see, such matchings are imperfect since the agents from $S$ are unmatched in every stable matching. To obtain a perfect matching, we must match some selection agent $s_{z} \in S$ to some vertex agent $v_{i}$, selecting the corresponding vertex $v_{i}$ into a solution for the input graph. This will incur two swaps to make the resulting matching stable. Below, we introduce edge agents and add them to the preference lists of agents $v_{i}$ and $y_{i}$, in order to ensure that the selected vertices induce an independent set.

Figure 2 depicts the crucial part of the induced acceptability graph for an edge $e_{\ell}=\left\{v_{i}, v_{j}\right\} \in E(G)$ with $i<j$. The weights at both sides of the edges denote the ranks of the respective endpoint towards the other endpoint.

To complete the construction, define the total number of swap as $\mathrm{d}_{\mathrm{G}}=2 k$. Clearly, the construction can be done in polynomial time. Now observe that every stable matching from the constructed profile does not match exactly $2 k$ agents, namely those from $T \cup S$.

To show that our construction is indeed a parameterized reduction it remains to show that $G$ admits a $k$-vertex independent set if and only if there exists a preference profile $P^{\prime}$ with $\tau\left(P, P^{\prime}\right) \leq$ $d=2 k$ which admits a perfect stable matching $M$.

For the "only if" part, assume that there exists a $k$-vertex independent set $V^{\prime} \subseteq V$ in $G$. We define the preference profile $P^{\prime}$ by performing the following $\mathrm{d}_{\mathrm{G}}=2 k$ swaps which involve the agents from $W \cup X$ that correspond to the vertices from the independent set. For each $z \in[k]$, swap the two agents $y_{i}$ and $x_{i}$ in the preference list of agent $w_{i}$, and swap the agents $v_{i}$ and $w_{i}$ in the preference list of agent $x_{i}$.

Now, we construct the following perfect matching $M$ for $P^{\prime}$. Let $V^{\prime}=\left\{v_{i_{1}}, v_{i_{2}}, \ldots, v_{i_{k}}\right\}$, where $i_{1}<i_{2}<\ldots<i_{k}$.

1. Put $\left\{h_{1}, h_{2}\right\} \in M$.

2. For each $z \in[k]$, put $\left\{v_{i_{z}}, s_{z}\right\},\left\{w_{i_{z}}, x_{i_{z}}\right\},\left\{t_{z}, y_{i_{z}}\right\} \in M$.

3. For each $i \in[n] \backslash\left\{i_{1}, i_{2}, \ldots, i_{k}\right\}$, put $\left\{v_{i}, x_{i}\right\},\left\{w_{i}, y_{i}\right\} \in M$. 
4. For each edge $e_{\ell} \in E(G)$, let $v_{i}$ and $v_{j}$ be the two endpoints of edge $e_{\ell}$ with $i<j$, and do the following. Recall that we have created two agents with the names $f_{\ell}^{v_{i}}$ and $e_{\ell}^{v_{j}}$. If $v_{i} \in V^{\prime}$ belongs to the independent set, implying that $v_{j} \in V \backslash V^{\prime}$, then put $\left\{e_{\ell}, f_{\ell}\right\},\left\{e_{\ell}^{y_{i}}, f_{\ell}^{v_{j}}\right\} \in M$. Otherwise, that is, if $v_{i} \notin V^{\prime}$, then put $\left\{e_{\ell}, f_{\ell}^{v_{i}}\right\},\left\{e_{\ell}^{y_{j}}, f_{\ell}\right\} \in M$.

Clearly, $M$ is perfect. We claim that $M$ is also stable for $P^{\prime}$.

Suppose, for the sake of contradiction, that $p$ is a blocking pair of $M$ for the profile $P^{\prime}$. First, observe that $p$ cannot involve $h_{1}, h_{2}, w_{i}$, or $x_{i}$ for any $i \in[n]$ as these agents already obtain their most preferred agents in $P^{\prime}$. For the same reason, $p$ cannot involve any agent $v_{i}$ for some $i \in$ $[n] \backslash\left\{i_{1}, i_{2}, \ldots, i_{k}\right\}$. Further, $p$ cannot involve an agent $s_{z}$ or an agent $t_{z}$ for any $z \in[k]$ because of the following. For each agent $c$ such that agent $s_{z}$ prefers $c$ to $M\left(s_{z}\right)=v_{i_{z}}$ we have that $c$ equals either some agent $v_{i}$ with $i \in[n] \backslash\left\{i_{1}, i_{2}, \ldots, i_{k}\right\}$, which already obtains her most preferred partner $M\left(v_{i}\right)=x_{i}$, or $c$ equals some agent $v_{i_{r}}$ with $r<z$, which prefers her partner $M\left(v_{i_{r}}\right)=s_{i_{r}}$ to agent $s_{i_{z}}$. Using a similar reasoning, we thus obtain that $p$ can neither involve an agent $t_{z}$, $z \in[k]$. Moreover, $p$ cannot involve two edge agents which correspond to two different edges or one vertex agent and one edge agent such that the corresponding vertex and edge are not incident to each other. Combining all of the above observations, we infer that $p$ either (a) involves two edge agents which correspond to the same edge or (b) a vertex agent and an edge agent such that the corresponding vertex and edge are not incident to each other.

For Case (a), observe that in each pair of mutually acceptable edge agents that correspond to the same edge, one receives its most-preferred partner in $M$ with respect to $P^{\prime}$. Hence, Case (b) must hold.

Let $e_{\ell}$ be the edge corresponding to the edge agent involved in $p$ and let $v_{i}$ and $v_{j}$ be the two endpoints of edge $e_{\ell}$ with $i<j$. Thus, $p=\left\{e_{\ell}^{y_{j}}, y_{j}\right\}$ or $p=\left\{v_{i}, f_{\ell}^{v_{i}}\right\}$.

If $p=\left\{e_{\ell}^{y_{j}}, y_{j}\right\}$, by the definition of blocking pairs, it follows that $M\left(e_{\ell}^{y_{j}}\right)=f_{\ell}^{v_{i}}$ and $M\left(y_{j}\right)=t_{z}$ for some $z \in[k]$. However, by the definition of $M$, from $M\left(y_{j}\right)=t_{z}$ we infer that $v_{j} \in V^{\prime}$ and from $M\left(e_{\ell}^{y_{j}}\right)=f_{\ell}^{v_{i}}$ we infer $v_{j} \in V \backslash V^{\prime}$, a contradiction.

Analogously, if $p=\left\{v_{i}, f_{\ell}^{v_{i}}\right\}$, by the definition of blocking pairs, it follows that $M\left(v_{i}\right)=s_{z}$ for some $z \in[k]$ and that $M\left(f_{\ell}^{v_{i}}\right)=e_{\ell}$. However, by our definition of $M$, from $M\left(v_{i}\right)=s_{z}$ we infer $v_{i} \in V^{\prime}$ and from $M\left(f_{\ell}^{v_{i}}\right)=e_{\ell}$ we infer $v_{i} \in V \backslash V^{\prime}$, a contradiction.

Hence, indeed, $M$ is stable in $P^{\prime}$.

For the "if" part, assume that there exists a perfect matching $M$ for profile $P$ and there exists a preference profile $P^{\prime}$ with $\tau\left(P, P^{\prime}\right) \leq d=2 k$ such that $M$ is stable for $P^{\prime}$. By the perfectness of $M$ there are $i_{1}, \ldots, i_{k} \in[n]$ such that for each $z \in[k]$ we have that $M\left(v_{i_{z}}\right) \in S$. We show that the vertex subset $V^{\prime}:=\left\{v_{i_{1}}, \ldots, v_{i_{k}}\right\}$ is a $k$-vertex independent set in $G$.

First of all, we claim the following.

Claim 4. For each agent $v_{i} \in V$ it holds that $M\left(v_{i}\right) \in S$ if and only if $M\left(y_{i}\right) \in T$. Moreover, no agent in $V \cup Y \cup E_{Y} \cup F_{V}$ changes her preference list in $P^{\prime}$.

Proof. We define two subsets $I_{1}:=\left\{i \in[n] \mid M\left(v_{i}\right) \in S\right\}$ and $I_{2}:=\left\{i^{\prime} \in[n] \mid M\left(y_{i^{\prime}}\right) \in T\right\}$. To show the first statement, it suffices to show that $I_{1}=I_{2}$. Clearly, $\left|I_{1}\right|=\left|I_{2}\right|=k$ because $|S|=|T|=k$ and $M$ is a perfect matching.

If we can show that for each $i \in I_{1} \cup I_{2}$ there are exactly two distinct swaps in $P^{\prime}$ in comparison to $P$, exactly one swap in $x_{i}$ 's preference list and exactly one swap in $w_{i}$ 's preference list, then by the swap budget $\mathrm{d}_{\mathrm{G}}=2 k$ and by the cardinalities of $I_{1}$ and $I_{2}$, it follows that $I_{1}=I_{2}$. 
Now, consider an index $i \in I_{1}$; the case when $i \in I_{2}$ is symmetric and omitted. By our definition of $I_{1}$, we have that $M\left(v_{i}\right) \in S$. Since $M$ is perfect, it follows that $\left\{x_{i}, w_{i}\right\} \in M$. Since $x_{i}$ and $v_{i}$ are each other's most preferred agent in profile $P$, at least one swap occurs in the preference lists of $x_{i}$ and $v_{i}$ to make $M$ stable for $P^{\prime}$; otherwise they would form a blocking pair. Similarly, since $w_{i}$ and $y_{i}$ are each other's most preferred agent in $P^{\prime}$, at least one swap occurs in the preference lists of $w_{i}$ or $y_{i}$ to make $M$ stable for $P^{\prime}$. Since $\left|I_{1}\right|=k$, meaning that there are at least $k$ distinct agents in $V$ matched to agents in $S$, the preference lists of $x_{i}$ and $v_{i}$ are affected indeed by exactly one swap (otherwise there would be more than $2 k$ swaps in total). Analogously, exactly one swap occurs in the preference lists of $w_{i}$ and $y_{i}$. By our swap budget, it follows that only agents in $V \cup X \cup W \cup Y$ may have a different preference list in $P^{\prime}$ compared to $P$. This, in particular, implies that $M\left(h_{1}\right)=M\left(h_{2}\right)$ as otherwise we need at least one more swap to make $M$ stable in $P^{\prime}$.

Observe that at least one agent, namely $h_{2}$, is between $v_{i}$ 's partner $M\left(v_{i}\right) \in S$ and $x_{i}$. Thus, it takes more than one swap to make $M$ stable in $P^{\prime}$ if we change the preference list of $v_{i}$ and not the preference list of $x_{i}$. Analogously, at least one agent, namely $h_{1}$, is between $y_{i}$ 's partner $M\left(y_{i}\right)$ and $w_{i}$; recall that we have just reasoned that $M\left(h_{1}\right)=h_{2}$. Thus, it also takes more than one swap to make $M$ stable in $P^{\prime}$ if we change the preference list of $y_{i}$ and not that of $w_{i}$ Summarizing, to make $M$ stable for $P^{\prime}$, there is exactly one swap in the preference list of $x_{i}$ and there is exactly one swap in the preference list of $w_{i}$.

The second statement follows directly from our swap budget and from the above reasoning that for each $i \in I_{1}$, there is exactly one swap in the preference list of $x_{i}$ and there is exactly one swap in the preference list of $w_{i}$ that are performed for $P$ to obtain $P^{\prime}$.

(of Claim 4) $\diamond$

To show that $V^{\prime}$ is indeed an independent set, suppose, towards a contradiction, that $V^{\prime}$ contain two adjacent vertices $v_{i}$ and $v_{j}$ with $i<j$. Let $e_{\ell}=\left\{v_{i}, v_{j}\right\}$ be the incident edge.

By the first statement in Claim 4, it follows that $M\left(y_{j}\right) \in T$.

By the second statement in Claim 4 , agent $v_{i}$ does not change her preference list in $P^{\prime}$, meaning that agent $v_{i}$ prefers $f_{\ell}^{v_{i}}$ to her partner $M\left(v_{i}\right) \in S$ in $P^{\prime}$. By the stability of $M$, it follows that $f_{\ell}^{v_{i}}$ prefers her partner $M\left(f_{\ell}^{v_{i}}\right)$ to $v_{i}$. Again, by the second statement in Claim 4 , agent $f_{\ell}^{v_{i}}$ does not change her preference list in $P^{\prime}$, meaning that $f_{\ell}^{v_{i}}$ prefers only $e_{\ell}^{y_{j}}$ to $v_{i}$. By the stability of $M$, we have $M\left(f_{\ell}^{v_{i}}\right)=e_{\ell}^{y_{j}}$. This implies that $\left\{e_{\ell}^{y_{j}}, y_{j}\right\}$ is a blocking pair, because $M\left(y_{j}\right) \in T$ by the above and by the second statement of Claim 4. This is a contradiction. Thus, indeed $I^{\prime}$ is a $k$-vertex independent set. The correctness follows.

The fact that an $n^{o\left(\mathrm{~d}_{\mathrm{G}}\right)}$-time algorithm for GLOBAL-NEAR+PERF would contradict the Exponential Time Hypothesis follows from this reduction in conjunction with the fact that an $n^{o(k)}$-time algorithm for IndePendent SET would contradict the Exponential Time Hypothesis [10].

The egalitarian case. To show the desired statements for the egalitarian case, we use the same idea of constructing type-two auxiliary agents in the proof of Theorem 5.3: We append a sufficiently large number $\Delta$ (to be specified later) of auxiliary agents to the end of each preference list which we constructed for the perfectness case such that the following conditions are satisfied. First, it is possible to match all auxiliary agents in pairs without inducing any egalitarian cost. Second, the matching $M$ constructed from an independent set as above has egalitarian cost at most $\Delta-1$. Third, every globally $\mathrm{d}_{\mathrm{G}}$-nearly stable matching within egalitarian cost at most $\Delta-1$ must be perfect. As mentioned, this can be done using the same construction as for the type-two auxiliary agents in Theorem 5.3 , adjusting the number of agents such that we add $\Delta:=$ $(2 k+1) \cdot\left(|T|+|V|+|W|+|E|+\left|E_{Y}\right|+1\right)+2 k=(2 k+1) \cdot(3 n+2 m+1)+2 k$ agents to the end of 
each preference list. The correctness follows in an analogous way as we prove Claim 2(1)-(2) and Claim 3.

By Proposition 2.3, the set of unmatched agents is the same across all stable matchings of a given preference profile $P$. We call an agent initially unmatched if she is not contained in any stable matching of the initial profile; otherwise she is initially matched. From Theorem 2.6 it follows that, in order to assign partners to the $\mathrm{n}_{\mathrm{u}}$ initially unmatched agents, we need to allow for at least $\mathrm{d}_{\mathrm{G}} \geq \mathrm{n}_{\mathrm{u}} / 2$ swaps in GLOBAL-NEAR+PERF. The number $\mathrm{n}_{\mathrm{u}}$ is thus a smaller parameter than $\mathrm{d}_{\mathrm{G}}$, meaning that it could be harder to obtain parameterized tractability result with respect to $n_{u}$ than to $\mathrm{d}_{\mathrm{G}}$. Indeed, we obtain intractability for $\mathrm{n}_{\mathrm{u}}$.

Corollary 5.6. Global-Near+Perf and Global-Near+Egal are W[1]-hard with respect to the number of initially unmatched $\mathrm{n}_{\mathrm{u}}$ agents. This also holds for LOCAL-NEAR+PERF and LOCAL$\mathrm{NEAR}+\mathrm{EGAL}$, even if $\mathrm{d}_{\mathrm{L}}=1$.

Proof. The reduction in the proof of Theorem 5.3 indeed is a parameterized reduction with respect to the number of unmatched agents which shows W[1]-hardness: First, observe that the reduction runs in polynomial time. Second, InDEPEndent SET is W[1]-hard with respect to the number $k$ of vertices in the sought independent set. Third, the number of unmatched agents in any stable matching of the initial profile $P$ constructed by the reduction is at most $2 k$. To see this, observe the following. Let $M$ be a stable matching for $P$. For each vertex agent $v_{i} \in V$, it must hold that $\left\{v_{i}, w_{i}\right\} \in M$, since this pair would otherwise block $M$. Thus, for each edge $e_{\ell}=\left\{v_{i}, v_{j}\right\} \in E(G)$, the edge agent $e_{\ell}$ is matched by $M$ to either $e_{\ell}^{v_{i}}$ or $e_{\ell}^{v_{j}}$, and $f_{\ell}$ is matched to $\left\{e_{\ell}^{v_{i}}, e_{\ell}^{v_{j}}\right\} \backslash\left\{M\left(e_{\ell}\right)\right\}$. Furthermore, for each $z \in[n-k]$, agent $t_{z} \in T$ is matched to agent $r_{z} \in R$, saturating $R$. Hence, the only unmatched agents are the $k$ agents in $\left\{t_{n-k+1}, \ldots, t_{n}\right\}$ and the $k$ agents in $S$.

On a side note, it is not hard to obtain a fixed-parameter algorithm for GLOBAL-NEAR+PERF with respect to the number $\mathrm{n}_{\mathrm{m}}$ of initially matched agents, that is, the number of agents that occur in every stable matching of the initial profile.

Proposition 5.7. GLOBAL-NEAR+PERF can be solved in $O\left(\mathrm{n}_{\mathrm{m}}{ }^{\mathrm{n}_{m}} \cdot \log \left(\mathrm{n}_{\mathrm{m}}\right) \cdot n^{2}\right)$ time and admits a problem kernel with $2 \mathrm{n}_{\mathrm{m}}$ agents that can be computed in linear time.

Proof Sketch. Let $P$ be a preference profile with agents sets $U$ and $W$ of size $n$ each. Further let $n_{1}$ (resp. $n_{2}$ ) denote the number of agents from $U$ that are initially matched (resp. unmatched) under any stable matching of $P$. Analogously, we define $n_{3}$ and $n_{4}$ for the set $W$, i.e. $n_{3}$ (resp. $n_{4}$ ) denotes the number of from $W$ that are initially matched (resp. unmatched) under any stable matching of $P$. By the definition of matching, it follows that

$$
n_{1}=n_{3} \text { and } n_{2}=n_{4} .
$$

Hence, together with the definition of $n_{m}$ and $n_{u}$, it follows that

$$
n_{1}+n_{3}=2 n_{1}=2 n_{3}=\mathrm{n}_{\mathrm{m}} \text { and } n_{2}+n_{4}=2 n_{2}=2 n_{4}=\mathrm{n}_{\mathrm{u}}
$$

Observe that no two initially unmatched agents are acceptable to each other since, if they were, then they would form a blocking pair. Thus, in each matching that represents a solution to GLOBALNEAR+PERF, each initially unmatched agent from $U$ (resp. $W$ ) is partnered with an initially 
matched agent from $W$ (resp. $U$ ). Thus, if $n_{2}>n_{3}$ or $n_{4}>n_{1}$, we can immediately return no (or a trivial no-instance). By (8), we obtain that

$$
|U|=n_{1}+n_{2} \leq n_{1}+n_{3}=\mathrm{n}_{\mathrm{m}} \text { and }|W|=n_{3}+n_{4} \leq n_{3}+n_{1}=\mathrm{n}_{\mathrm{m}} .
$$

In other words, we obtain a problem kernel with at most $2 \mathrm{n}_{\mathrm{m}}$ agents. This takes $O\left(n^{2}\right)$ time.

To solve GLOBAL-NEAR+PERF in $O\left(2^{n_{m}} \cdot \log \left(\mathrm{n}_{\mathrm{m}}\right) \cdot n^{2}\right)$ time, observe that by (9) each agent from $U \cup W$ has at most $\mathrm{n}_{\mathrm{m}}$ agents in her preference list, and there are $2 \mathrm{n}_{\mathrm{m}}$ agents from $U \cup W$. We hence iterate through all $\left(\mathrm{n}_{\mathrm{m}} !\right)^{2 \mathrm{n}_{\mathrm{m}}}$ target profile $P^{\prime}$ which differs from $P$ by at most $\mathrm{d}_{\mathrm{G}}$ swaps. For each of these preference profiles, we check in $O\left(n^{2}\right)$ time whether it admits a stable and perfect matching.

We conclude this section by remarking that the kernelization approach for Proposition 5.7 cannot be directly adapted to work for the egalitarian case because not every initially unmatched agent needs to be matched in an optimal egalitarian stable matching.

\section{Conclusion and Open Questions}

In this paper we have introduced and studied a framework describing the strength of the stability of matchings under preferences. Our framework unifies and extends some of the few approaches that already exist in the literature, such as additive $\alpha$-stability [32, 1], $r$-maximal stability [12], and robustness to the errors in inputs [23, 24]. We have elaborated that all these approaches can be expressed by the same model, where the central idea is to investigate the preference profiles which have bounded distance to the input profile. Thus, we open up a general framework to study questions that have already received attention in the literature.

From a computational point of view, we have shown a somehow counter-intuitive relation between strength of stability, and other criteria of social optimality. On the one hand, there exists a polynomial-time algorithm for finding robust matchings if they exist (recall that robustness is a stronger concept than classic stability) even if we additionally aim to reach social optimality. On the other hand, if we ask about near stability instead of robustness, the problem becomes computationally hard in many aspects: it is hard to approximate and hard from the point of view of parameterized complexity. Our computational results are summarized in Table 1.

We conclude with some challenges for future research. First of all, for the case where no $d$-robust matchings exist, we may look for a matching that admits the fewest number of blocking pairs [3, 8] in every profile that has swap distance $d$ to the input profile.

Second, continuing our research in Section 4 where we showed that Robust MATCHING becomes NP-hard when ties are allowed, our near stability concept can be generalized to the case with ties. Moreover, both robustness and near stability, though introduced for the bipartite variant (STABLE MARRIAGe), can be generalized to the non-bipartite variant (Stable RoOmmates). It would be interesting to see whether our algorithmic results transfer to these cases.

Regarding preference restrictions [5], it would be interesting to know whether assuming a special preference structure can help in finding tractable cases for nearly stable matchings.

\section{Acknowledgments}

Piotr Skowron was supported by the Foundation for Polish Science within the Homing programme (Project title: "Normative Comparison of Multiwinner Election Rules"). Jiehua Chen and Manuel 
Sorge were supported by the European Research Council (ERC) under the European Union's Horizon 2020 research and innovation programme under grant agreement numbers 677651 (JC) and $714704(\mathrm{MS})$.

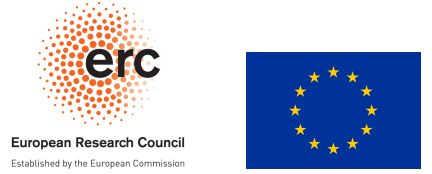

\section{References}

[1] E. Anshelevich, S. Das, and Y. Naamad. Anarchy, stability, and utopia: Creating better matchings. Autonomous Agents and Multi-Agent Systems, 26(1):120-140, 2013. 6, 44

[2] H. Aziz, P. Biró, S. Gaspers, R. de Haan, N. Mattei, and B. Rastegari. Stable matching with uncertain linear preferences. In Proc. SAGT-16, pages 195-206, 2016. 5

[3] P. Biró, D. Manlove, and E. McDermid. "Almost stable" matchings in the Roommates problem with bounded preference lists. Theor. Comput. Sci., 432:10-20, 2012. 44

[4] J. Boudreau and V. Knoblauch. Preferences and the price of stability in matching markets. Theory and Decision, 74(4):565-589, 2013. 6

[5] R. Bredereck, J. Chen, U. P. Finnendahl, and R. Niedermeier. Stable roommate with narcissistic, single-peaked, and single-crossing preferences. In Proc. ADT-17, pages 315-330, 2017. 44

[6] R. Bredereck, P. Faliszewski, A. Kaczmarczyk, R. Niedermeier, P. Skowron, and N. Talmon. Robustness among multiwinner voting rules. In Proc. SAGT-17, pages 80-92, 2017. 6

[7] P. Camion. Characterization of totally unimodular matrices. Proceedings of the American Mathematical Society, 16(5):1068-1073, 1965. 28

[8] J. Chen, D. Hermelin, M. Sorge, and H. Yedidsion. How hard is it to satisfy (almost) all roommates? In Proc. ICALP-18, pages 35:1-35:15, 2018. 44

[9] J. Chen, R. Niedermeier, and P. Skowron. Stable marriage with multi-modal preferences. In Proc. EC-18, pages 269-286, 2018. 5

[10] M. Cygan, F. V. Fomin, L. Kowalik, D. Lokshtanov, D. Marx, M. Pilipczuk, M. Pilipczuk, and S. Saurabh. Parameterized Algorithms. Springer, 2015. 4, 39, 42

[11] R. G. Downey and M. R. Fellows. Fundamentals of Parameterized Complexity. Springer, 2013. 4

[12] J. Drummond and C. Boutilier. Elicitation and approximately stable matching with partial preferences. In Proc. IJCAI-13, pages 97-105, 2013. 6, 11, 44

[13] P. Faliszewski and J. Rothe. Control and bribery in voting. In Handbook of Computational Social Choice. Cambridge University Press, 2016. 6 
[14] J. Flum and M. Grohe. Parameterized Complexity Theory. Springer, 2006. 4

[15] D. Gale and L. S. Shapley. College admissions and the stability of marriage. The American Mathematical Monthly, 120(5):386-391, 1962. 1, 4, 8, 13, 38

[16] B. Genc, M. Siala, G. Simonin, and B. O'Sullivan. On the complexity of robust stable marriage. In Proc. COCOA-17, pages 441-448, 2017. 6

[17] B. Genc, M. Siala, G. Simonin, and B. O'Sullivan. Robust stable marriage. In Proc. AAAI-17, pages 4925-4926, 2017. 6

[18] D. Gusfield and R. W. Irving. The Stable Marriage Problem-Structure and Algorithms. Foundations of Computing Series. MIT Press, 1989. 4, 5, 6, 8, 13, 14, 15, 19, 24, 27

[19] R. W. Irving. Stable marriage and indifference. Discrete Applied Mathematics, 48(3):261-272, 1994. 6, 29

[20] R. W. Irving, P. Leather, and D. Gusfield. An efficient algorithm for the 'optimal' stable marriage. Journal of the ACM, 34(3):532-543, 1987. 3, 38

[21] M. Kendall. A new measure of rank correlation. Biometrika, 30:81-89, 1948. 7

[22] D. Knuth. Mariages Stables. Les Presses de L'Université de Montréal, 1976. 5

[23] T. Mai and V. V. Vazirani. Finding stable matchings that are robust to errors in the input. In Proc. ESA-18, pages 60:1-60:11, 2018. 5, 44

[24] T. Mai and V. V. Vazirani. A generalization of Birkhoff's theorem for distributive lattices, with applications to robust stable matchings. Technical report, arXiv:1804.05537 [cs.DM], 2018. 5, 44

[25] D. Manlove, R. W. Irving, K. Iwama, S. Miyazaki, and Y. Morita. Hard variants of stable marriage. Theor. Comput. Sci., 276(1-2):261-279, 2002. 4

[26] D. F. Manlove. Algorithmics of Matching Under Preferences, volume 2. WorldScientific, 2013. 5

[27] V. Menon and K. Larson. Robust and approximately stable marriages under partial information. In Proc. WINE-18, pages 341-355, 2018. 6

[28] G. Miller. The magical number seven, plus or minus two: Some limits on our capacity for processing information. Psychological Review, 63(2):81-97, 1956. 2

[29] S. Miyazaki and K. Okamoto. Jointly stable matchings. In Proc. ISAAC-17, pages 56:1-56:12, 2017. 5

[30] R. Niedermeier. Invitation to Fixed-Parameter Algorithms. Oxford University Press, 2006. 4

[31] C. H. Papadimitriou. The complexity of finding nash equilibria. In Algorithmic Game Theory, pages 29-51. Cambridge University Press, 2007. 3 
[32] M. S. Pini, F. Rossi, K. B. Venable, and T. Walsh. Stability, Optimality and Manipulation in Matching Problems with Weighted Preferences. Algorithms, 6(4):782-804, 2013. 6, 44

[33] A. Roth and M. Sotomayor. Two-Sided Matching: A Study in Game-Theoretic Modeling and Analysis. 1992. Part of Econometric Society Monographs. 5

[34] D. Shiryaev, L. Yu, and E. Elkind. Possible and necessary winners of partial tournaments. In Proc. AAMAS-13, pages 415-422, 2013. 6 